\title{
SUPPORTING INFORMATION \\ Determination of Absolute Stereochemistry of \\ Flexible Molecules Using a Vibrational Circular Dichroism (VCD) Spectra Alignment Algorithm
}

Lennard Böselt, Dominik Sidler, Tobias Kittelmann, Jürgen Stohner, Daniel Zindel, Trixie Wagner, Sereina Riniker*

[*] Laboratory of Physical Chemistry, ETH Zurich, Vladimir-Prelog-Weg 2, 8093 Zurich, Switzerland

Email: sriniker@ethz.ch

\section{Contents}

1 Conformationally Rigid Molecules $\quad$ S2

1.1 Performance with Automatic Peak Selection . . . . . . . . . . . . . . . . . . . S2

1.2 Performance with Manual Peak Selection . . . . . . . . . . . . . . . . . . . . s9

2 Semi-Rigid Molecules $\quad$ S16

2.1 Performance with Automatic Peak Selection . . . . . . . . . . . . . . . . . S16

2.2 Performance with Manual Peak Selection . . . . . . . . . . . . . . . . . . . S24

3 Conformationally Flexible Molecules - All Conformers S32

3.1 Performance with Automatic Peak Selection, No Upwards Shifts Allowed . . . . S32

3.2 Performance with Automatic Peak Selection, Upwards Shifts Allowed . . . . . . . S43

3.3 Performance with Manual Peak Selection, Upwards Shifts Allowed . . . . . . . . S54

3.4 Convergence . . . . . . . . . . . . . . . . . . . . . S65

4 Conformationally Flexible Molecules - 20 Conformers $\quad$ S69

4.1 Performance with Automatic Peak Selection, Upwards Shifts Allowed . . . . . . . S69 


\section{Conformationally Rigid Molecules}

\subsection{Performance with Automatic Peak Selection}
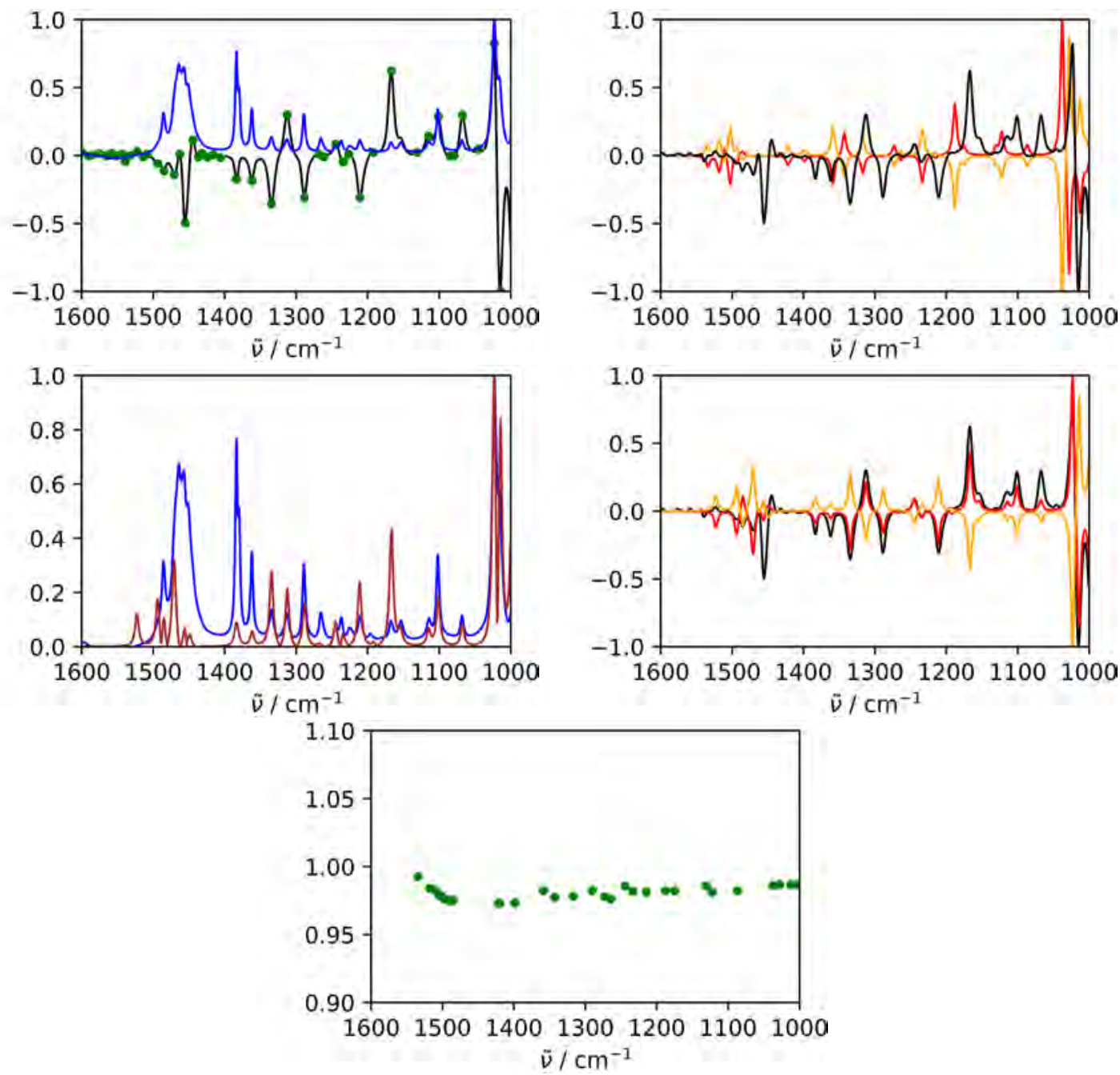

Figure S1: Compound 1, (+)-fenchone. (Top left): Experimental VCD spectrum (black) and IR spectrum (blue). The green dots mark the peaks picked by the automatic procedure. (Top right): Unprocessed theoretical VCD spectrum of the correct enantiomer (red) and the other enantiomer (orange), as well as the experimental VCD spectrum (black). (Middle left): Experimental IR spectrum (blue) and theoretical IR (brown) obtained by taking the absolute value of the aligned VCD spectrum. (Middle right): Aligned theoretical (red, orange) and experimental VCD spectra (black) using the VSA algorithm. The alignment was performed in the wavenumber range $[1000,1600] \mathrm{cm}^{-1}$. No upwards shifts were allowed. (Bottom): The shifting value of each theoretical peak determined by the VSA algorithm. 

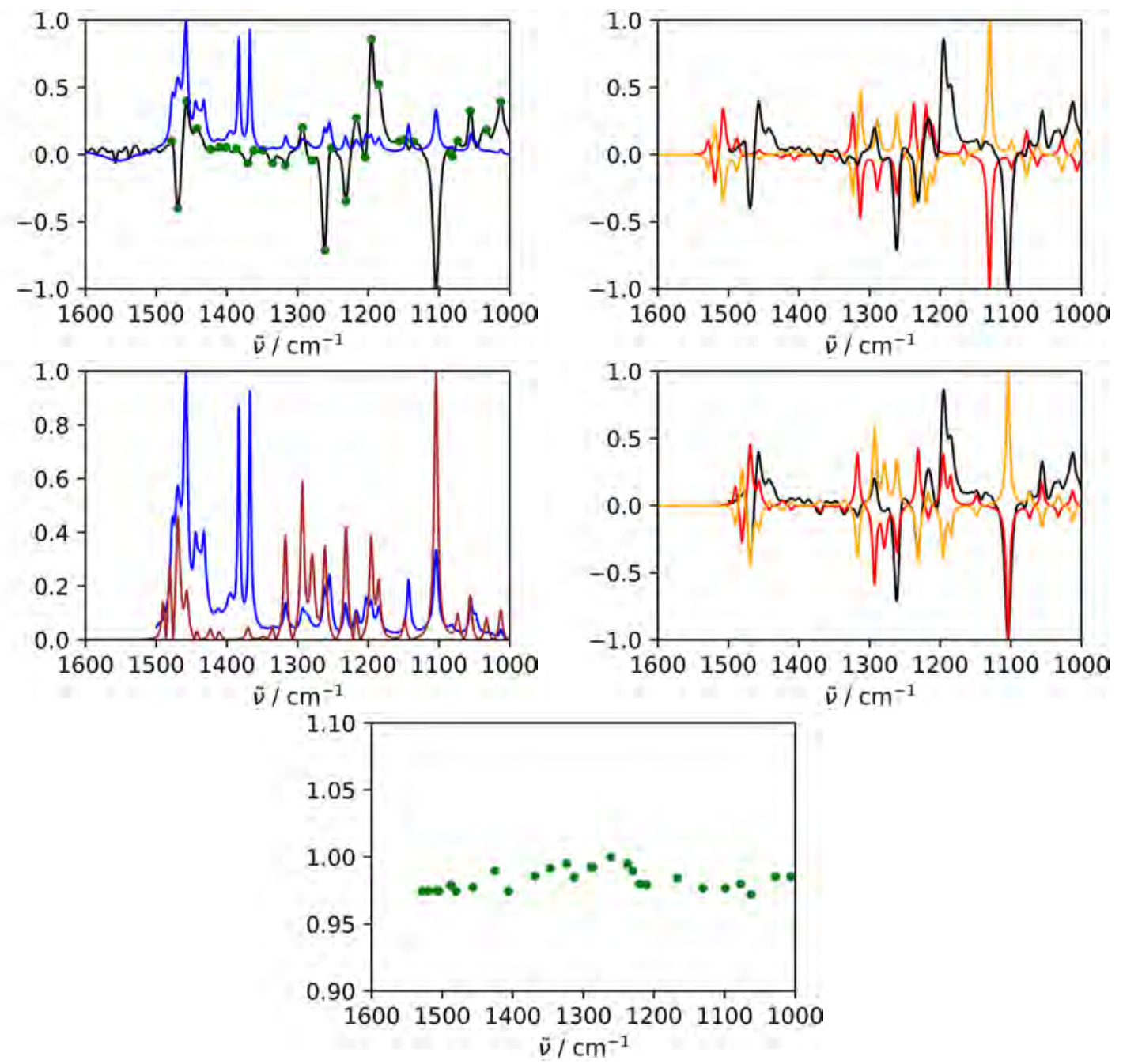

Figure S2: Compound 2, (-)- $\beta$-pinene. (Top left): Experimental VCD spectrum (black) and IR spectrum (blue). The green dots mark the peaks picked by the automatic procedure. (Top right): Unprocessed theoretical VCD spectrum of the correct enantiomer (red) and the other enantiomer (orange), as well as the experimental VCD spectrum (black). (Middle left): Experimental IR spectrum (blue) and theoretical IR (brown) obtained by taking the absolute value of the aligned VCD spectrum. (Middle right): Aligned theoretical (red, orange) and experimental VCD spectra (black) using the VSA algorithm. The alignment was performed in the wavenumber range $[1000,1500] \mathrm{cm}^{-1}$. No upwards shifts were allowed. (Bottom): The shifting value of each theoretical peak determined by the VSA algorithm. 



Figure S3: Compound 3, $(R)-(+)$-propylene oxide. (Top left): Experimental VCD spectrum (black) and IR spectrum (blue). The green dots mark the peaks picked by the automatic procedure. (Top right): Unprocessed theoretical VCD spectrum of the correct enantiomer (red) and the other enantiomer (orange), as well as the experimental VCD spectrum (black). (Middle left): Experimental IR spectrum (blue) and theoretical IR (brown) obtained by taking the absolute value of the aligned VCD spectrum. (Middle right): Aligned theoretical (red, orange) and experimental VCD spectra (black) using the VSA algorithm. The alignment was performed in the wavenumber range $[1025,1500] \mathrm{cm}^{-1}$. No upwards shifts were allowed. (Bottom): The shifting value of each theoretical peak determined by the VSA algorithm. 

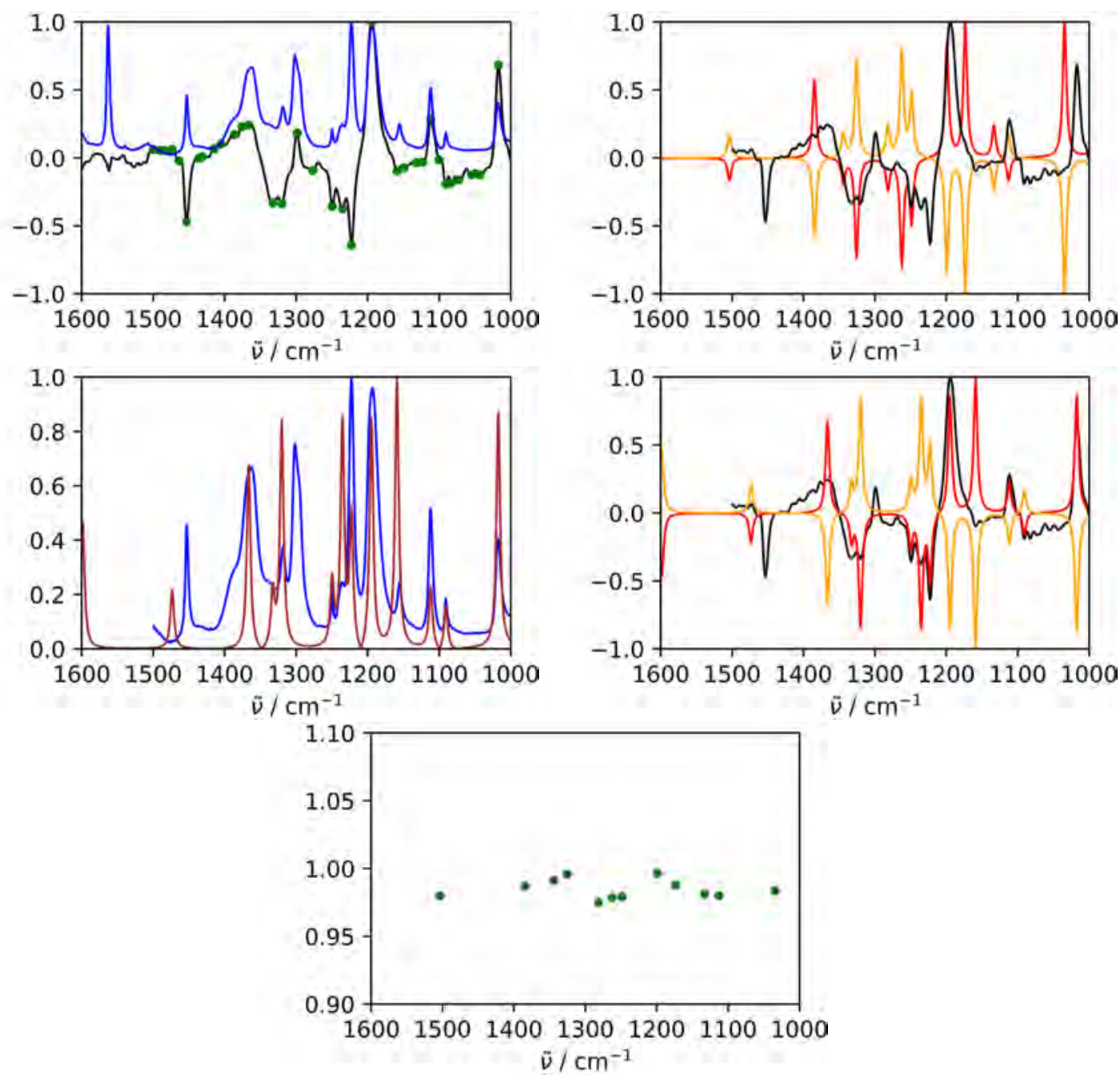

Figure S4: Compound 4, (1R)-(-)-2-azabicyclo[2.2.1]hept-5-en-3-one. (Top left): Experimental VCD spectrum (black) and IR spectrum (blue). The green dots mark the peaks picked by the automatic procedure. (Top right): Unprocessed theoretical VCD spectrum of the correct enantiomer (red) and the other enantiomer (orange), as well as the experimental VCD spectrum (black). (Middle left): Experimental IR spectrum (blue) and theoretical IR (brown) obtained by taking the absolute value of the aligned VCD spectrum. (Middle right): Aligned theoretical (red, orange) and experimental VCD spectra (black) using the VSA algorithm. The alignment was performed in the wavenumber range $[1000,1500] \mathrm{cm}^{-1}$. No upwards shifts were allowed. (Bottom): The shifting value of each theoretical peak determined by the VSA algorithm. 

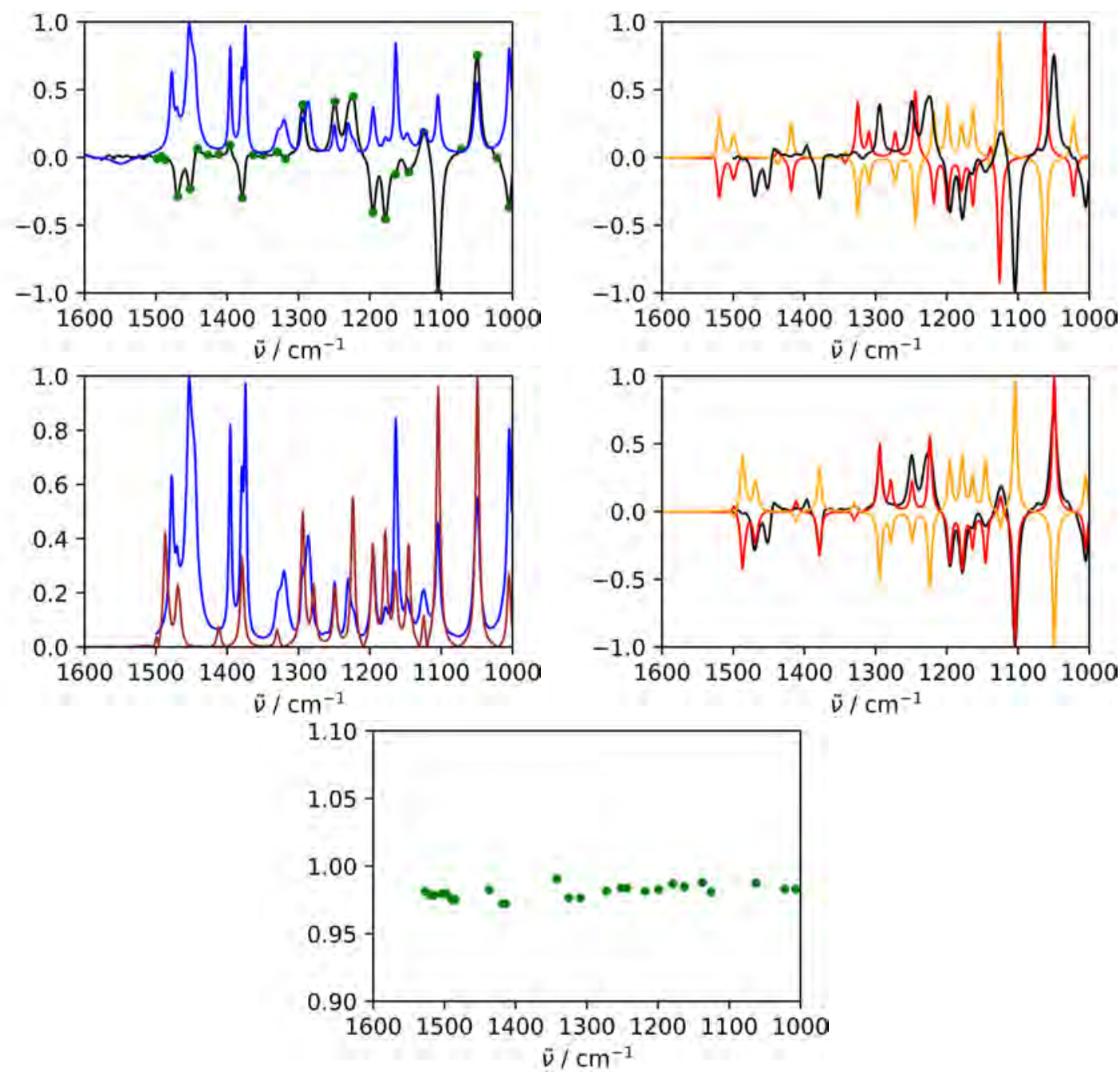

Figure S5: Compound 5, (1R)-(-)-camphorquinone. (Top left): Experimental VCD spectrum (black) and IR spectrum (blue). The green dots mark the peaks picked by the automatic procedure. (Top right): Unprocessed theoretical VCD spectrum of the correct enantiomer (red) and the other enantiomer (orange), as well as the experimental VCD spectrum (black). (Middle left): Experimental IR spectrum (blue) and theoretical IR (brown) obtained by taking the absolute value of the aligned VCD spectrum. (Middle right): Aligned theoretical (red, orange) and experimental VCD spectra (black) using the VSA algorithm. The alignment was performed in the wavenumber range $[1000,1500] \mathrm{cm}^{-1}$. No upwards shifts were allowed. (Bottom): The shifting value of each theoretical peak determined by the VSA algorithm. 

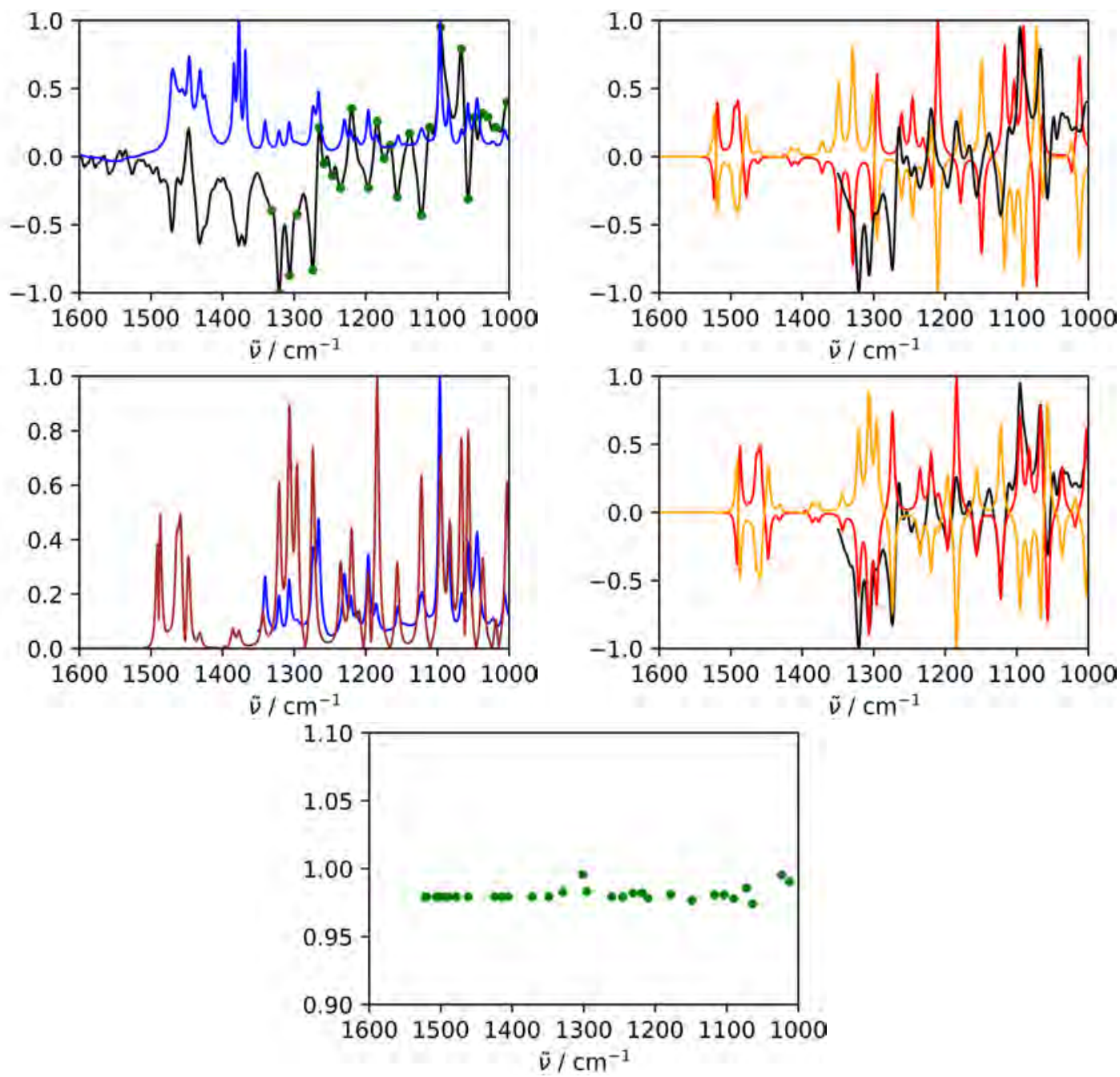

Figure S6: Compound 6, (-)- $\alpha$-pinene oxide. (Top left): Experimental VCD spectrum (black) and IR spectrum (blue). The green dots mark the peaks picked by the automatic procedure. (Top right): Unprocessed theoretical VCD spectrum of the correct enantiomer (red) and the other enantiomer (orange), as well as the experimental VCD spectrum (black). (Middle left): Experimental IR spectrum (blue) and theoretical IR (brown) obtained by taking the absolute value of the aligned VCD spectrum. (Middle right): Aligned theoretical (red, orange) and experimental VCD spectra (black) using the VSA algorithm. The alignment was performed in the wavenumber range $[1000,1350] \mathrm{cm}^{-1}$. No upwards shifts were allowed. (Bottom): The shifting value of each theoretical peak determined by the VSA algorithm. 



Figure S7: Compound 7, $(R)$-camphor. (Top left): Experimental VCD spectrum (black) and IR spectrum (blue) taken from Ref. [1]. The green dots mark the peaks picked by the automatic procedure. (Top right): Unprocessed theoretical VCD spectrum of the correct enantiomer (red) and the other enantiomer (orange), as well as the experimental VCD spectrum (black). (Middle left): Experimental IR spectrum (blue) and theoretical IR (brown) obtained by taking the absolute value of the aligned VCD spectrum. (Middle right): Aligned theoretical (red, orange) and experimental VCD spectra (black) using the VSA algorithm. The alignment was performed in the wavenumber range $[1000,1600] \mathrm{cm}^{-1}$. No upwards shifts were allowed. (Bottom): The shifting value of each theoretical peak determined by the VSA algorithm. 


\subsection{Performance with Manual Peak Selection}

Table S1: Performance of the VSA algorithm for conformationally rigid molecules as measured by the Pearson coefficients $r$ for the correct (c) and incorrect (ic) enantiomers. The alignment was performed with manually picked peaks.

\begin{tabular}{ccc}
\hline Molecule & $r_{\mathrm{c}}$ & $r_{\text {ic }}$ \\
\hline \hline $\mathbf{1}$ & 0.90 & -0.90 \\
\hline $\mathbf{2}$ & 0.60 & -0.60 \\
\hline $\mathbf{3}$ & 0.92 & -0.92 \\
\hline $\mathbf{4}$ & 0.68 & -0.68 \\
\hline $\mathbf{5}$ & 0.89 & -0.89 \\
\hline $\mathbf{6}$ & 0.75 & -0.75 \\
\hline $\mathbf{7}$ & 0.81 & -0.81 \\
\hline
\end{tabular}
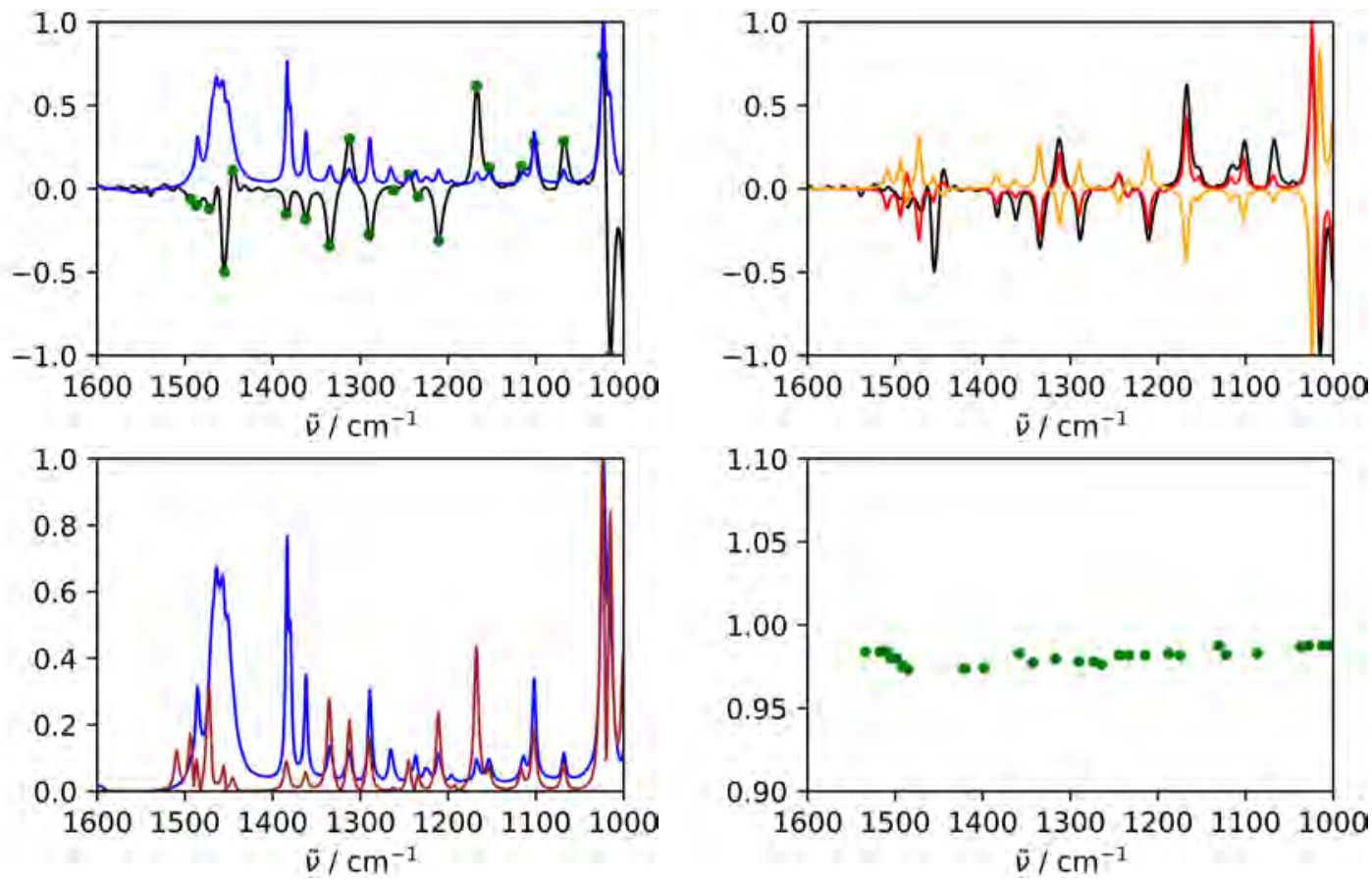

Figure S8: Compound 1, (+)-fenchone. (Top left): Experimental VCD spectrum (black) and IR spectrum (blue). The green dots mark the peaks picked manually. (Top right): Aligned theoretical VCD spectrum of the correct enantiomer (red) and the other enantiomer (orange) and experimental VCD spectra (black) using the VSA algorithm. The alignment was performed in the wavenumber range $[1000,1600] \mathrm{cm}^{-1}$. No upwards shifts were allowed. (Bottom left): Experimental IR spectrum (blue) and theoretical IR (brown) obtained by taking the absolute value of the aligned VCD spectrum. (Bottom right): The shifting value of each theoretical peak determined by the VSA algorithm. 

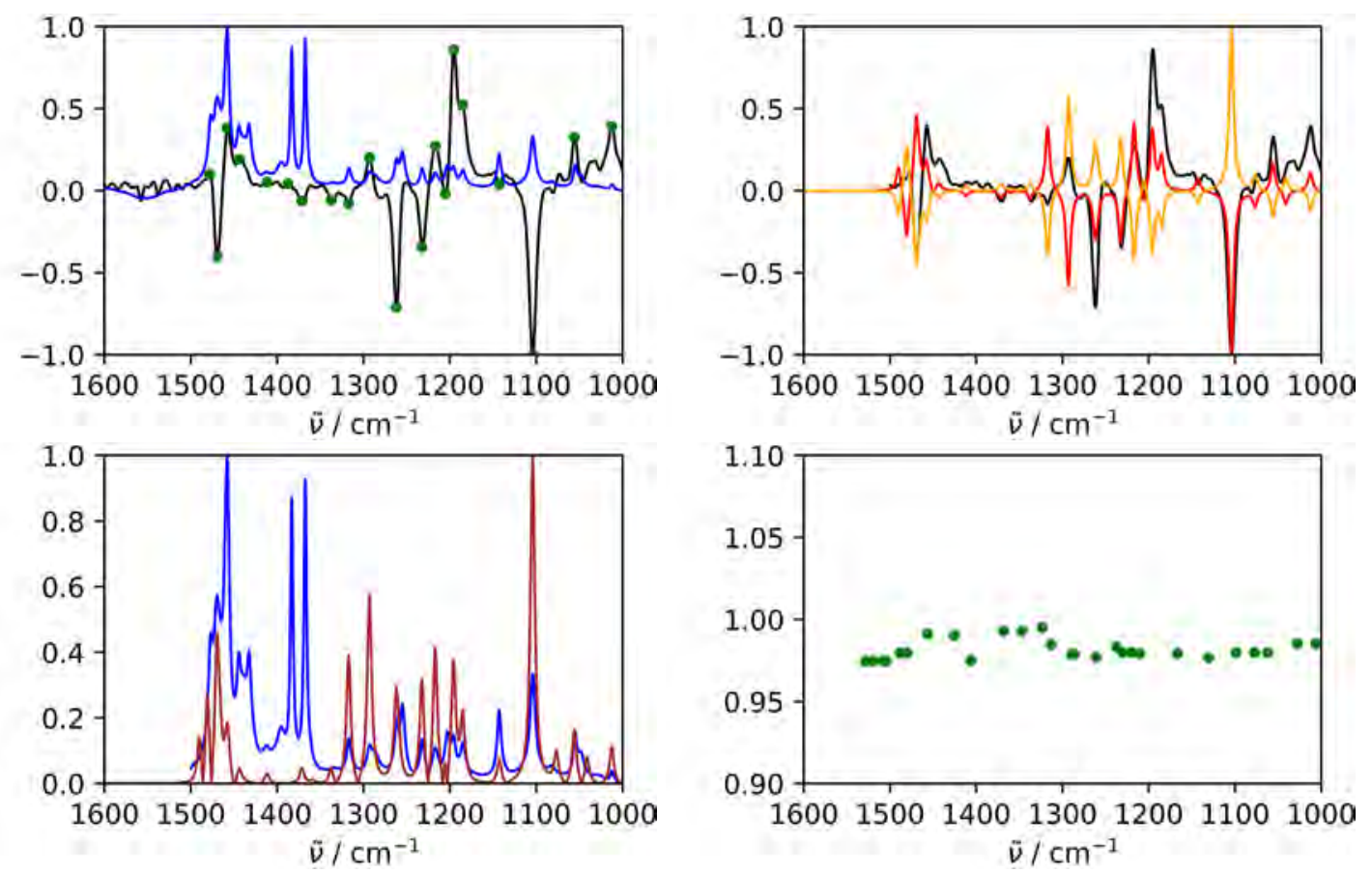

Figure S9: Compound 2, (-)- $\beta$-pinene. (Top left): Experimental VCD spectrum (black) and IR spectrum (blue). The green dots mark the peaks picked manually. (Top right): Aligned theoretical VCD spectrum of the correct enantiomer (red) and the other enantiomer (orange) and experimental VCD spectra (black) using the VSA algorithm. The alignment was performed in the wavenumber range $[1000,1500] \mathrm{cm}^{-1}$. No upwards shifts were allowed. (Bottom left): Experimental IR spectrum (blue) and theoretical IR (brown) obtained by taking the absolute value of the aligned VCD spectrum. (Bottom right): The shifting value of each theoretical peak determined by the VSA algorithm. 

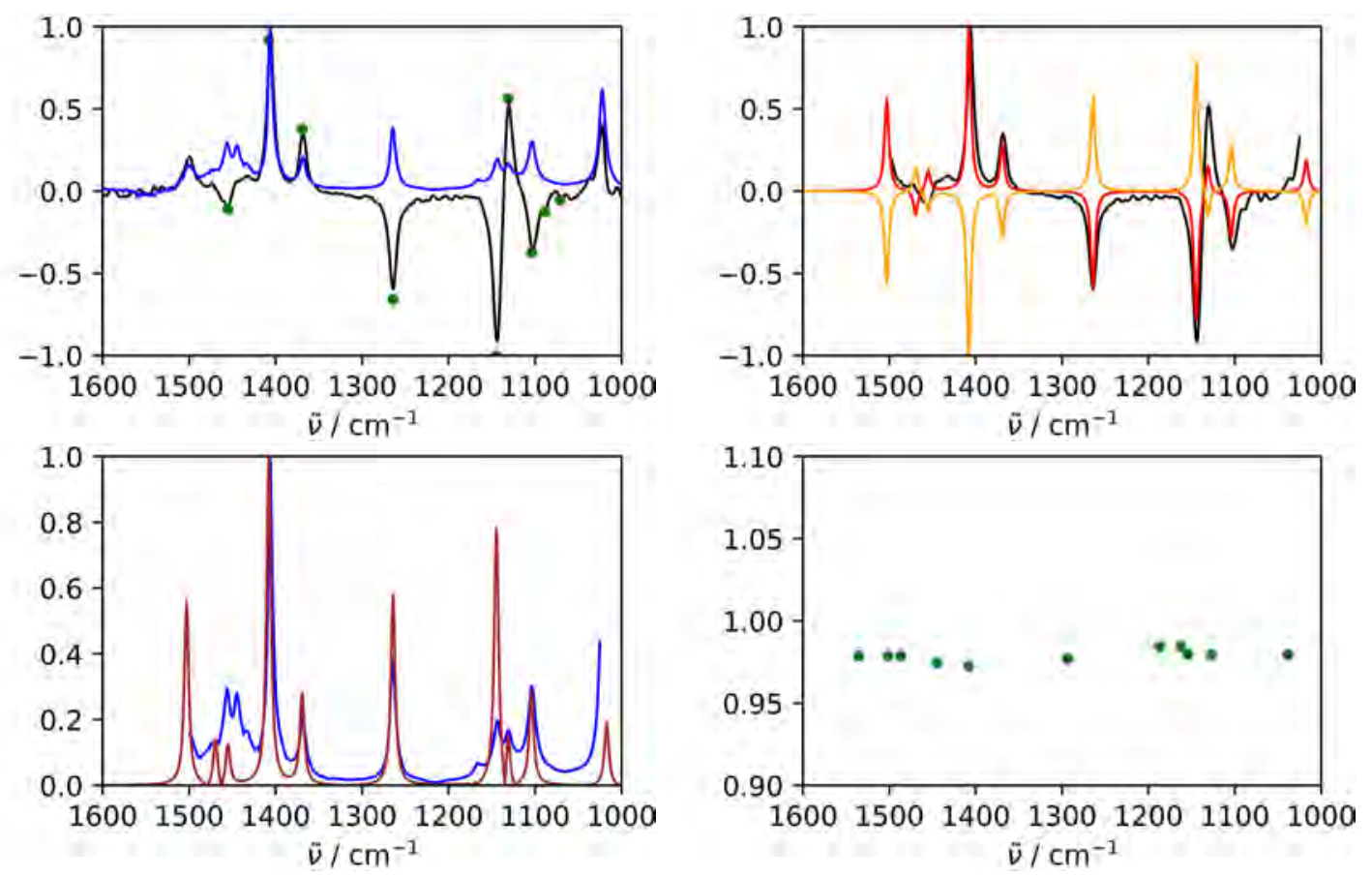

Figure S10: Compound 3, $(R)-(+)$-propylene oxide. (Top left): Experimental VCD spectrum (black) and IR spectrum (blue). The green dots mark the peaks picked manually. (Top right): Aligned theoretical VCD spectrum of the correct enantiomer (red) and the other enantiomer (orange) and experimental VCD spectra (black) using the VSA algorithm. The alignment was performed in the wavenumber range $[1025,1500] \mathrm{cm}^{-1}$. No upwards shifts were allowed. (Bottom left): Experimental IR spectrum (blue) and theoretical IR (brown) obtained by taking the absolute value of the aligned VCD spectrum. (Bottom right): The shifting value of each theoretical peak determined by the VSA algorithm. 

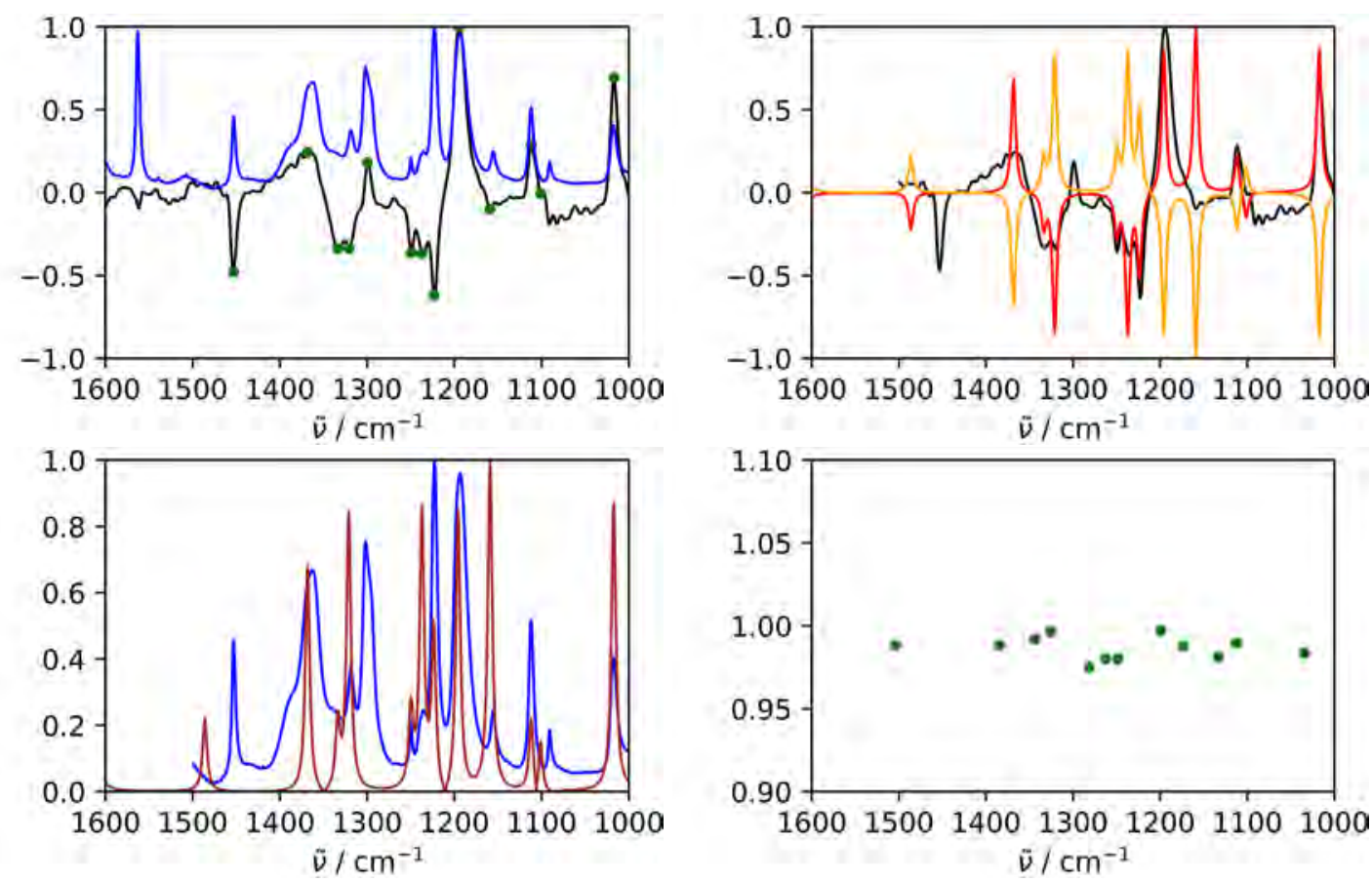

Figure S11: Compound 4, (1R)-(-)-2-azabicyclo[2.2.1]hept-5-en-3-one. (Top left): Experimental VCD spectrum (black) and IR spectrum (blue). The green dots mark the peaks picked manually. (Top right): Aligned theoretical VCD spectrum of the correct enantiomer (red) and the other enantiomer (orange) and experimental VCD spectra (black) using the VSA algorithm. The alignment was performed in the wavenumber range $[1000,1500] \mathrm{cm}^{-1}$. No upwards shifts were allowed. (Bottom left): Experimental IR spectrum (blue) and theoretical IR (brown) obtained by taking the absolute value of the aligned VCD spectrum. (Bottom right): The shifting value of each theoretical peak determined by the VSA algorithm. 

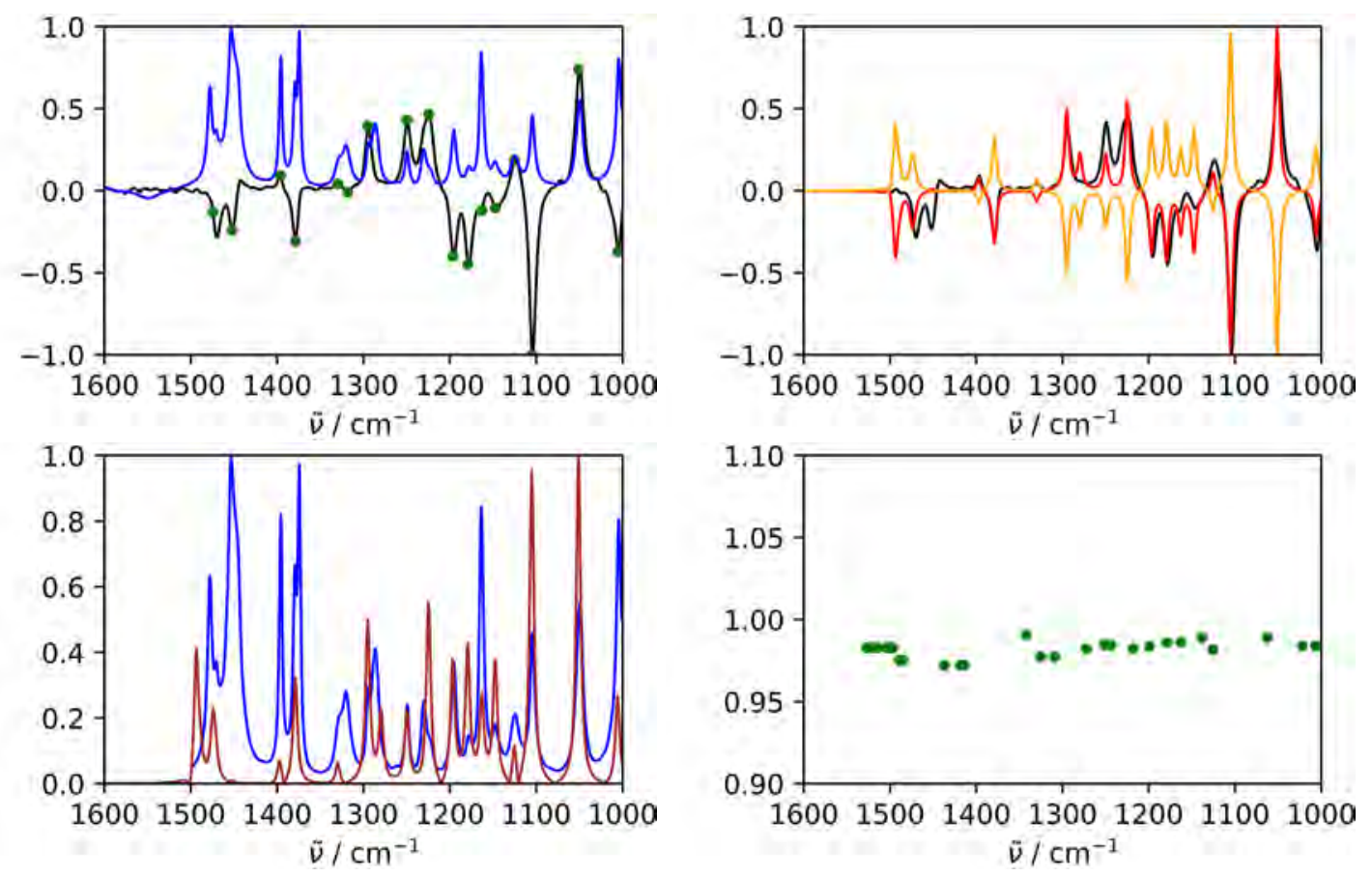

Figure S12: Compound 5, (1R)-(-)-camphorquinone. (Top left): Experimental VCD spectrum (black) and IR spectrum (blue). The green dots mark the peaks picked manually. (Top right): Aligned theoretical VCD spectrum of the correct enantiomer (red) and the other enantiomer (orange) and experimental VCD spectra (black) using the VSA algorithm. The alignment was performed in the wavenumber range $[1000,1500] \mathrm{cm}^{-1}$. No upwards shifts were allowed. (Bottom left): Experimental IR spectrum (blue) and theoretical IR (brown) obtained by taking the absolute value of the aligned VCD spectrum. (Bottom right): The shifting value of each theoretical peak determined by the VSA algorithm. 

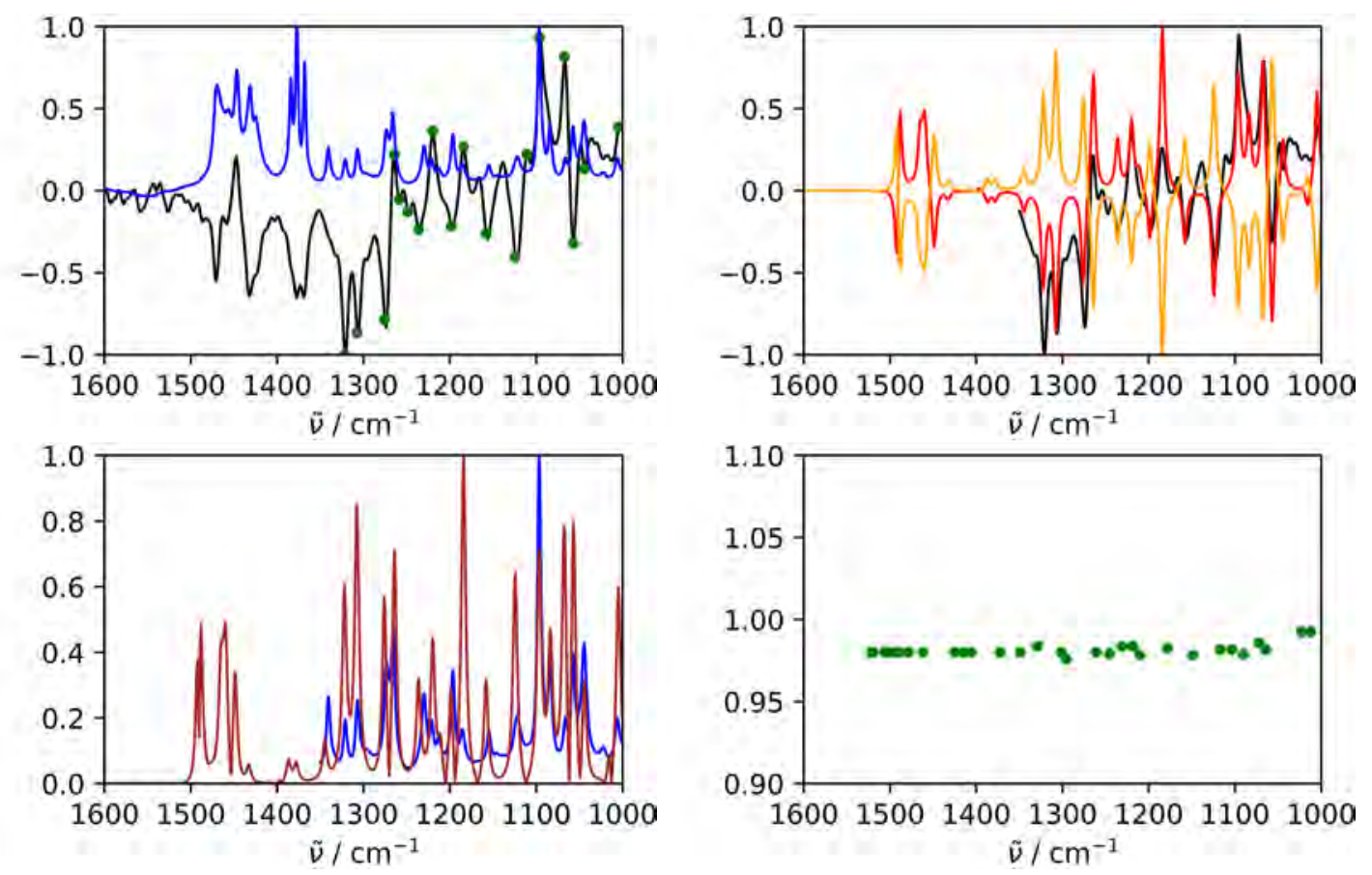

Figure S13: Compound 6, (-)- $\alpha$-pinene oxide. (Top left): Experimental VCD spectrum (black) and IR spectrum (blue). The green dots mark the peaks picked manually. (Top right): Aligned theoretical VCD spectrum of the correct enantiomer (red) and the other enantiomer (orange) and experimental VCD spectra (black) using the VSA algorithm. The alignment was performed in the wavenumber range $[1000,1350] \mathrm{cm}^{-1}$. No upwards shifts were allowed. (Bottom left): Experimental IR spectrum (blue) and theoretical IR (brown) obtained by taking the absolute value of the aligned VCD spectrum. (Bottom right): The shifting value of each theoretical peak determined by the VSA algorithm. 

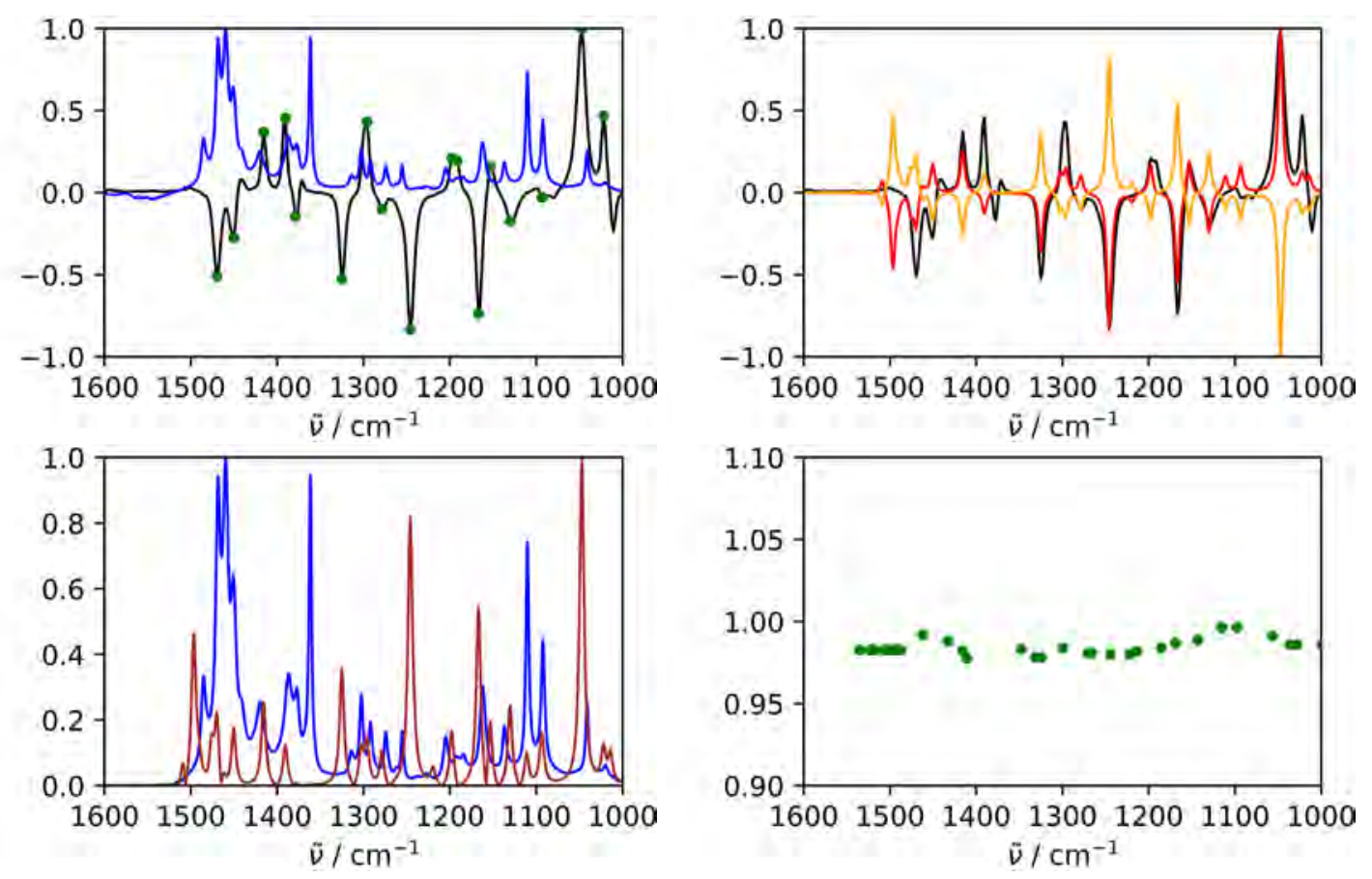

Figure S14: Compound 7, $(R)$-camphor. (Top left): Experimental VCD spectrum (black) and IR spectrum (blue) taken from Ref. [1]. The green dots mark the peaks picked manually. (Top right): Aligned theoretical VCD spectrum of the correct enantiomer (red) and the other enantiomer (orange) and experimental VCD spectra (black) using the VSA algorithm. The alignment was performed in the wavenumber range $[1000,1600] \mathrm{cm}^{-1}$. No upwards shifts were allowed. (Bottom left): Experimental IR spectrum (blue) and theoretical IR (brown) obtained by taking the absolute value of the aligned VCD spectrum. (Bottom right): The shifting value of each theoretical peak determined by the VSA algorithm. 


\section{Semi-Rigid Molecules}

\subsection{Performance with Automatic Peak Selection}
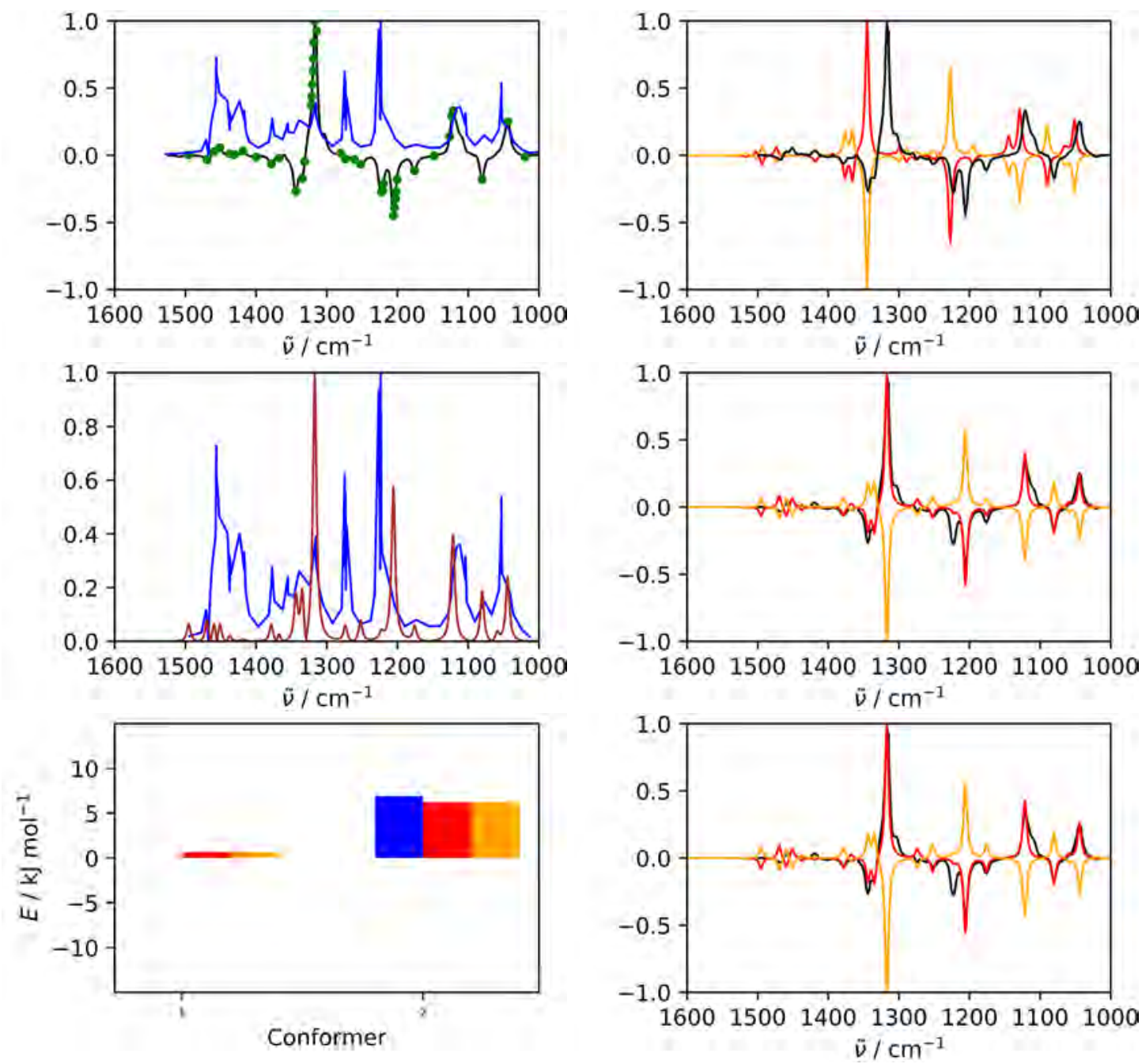

Figure S15: Compound 8, $(R)-(+)$-3-methylcyclohexanone. (Top left): Experimental VCD spectrum (black) and IR spectrum (blue) taken from Ref. [2]. The green dots mark the peaks picked by the automatic procedure. (Top right): Unprocessed theoretical VCD spectrum of the correct enantiomer (red) and the other enantiomer (orange), as well as the experimental VCD spectrum (black). (Middle left): Experimental IR spectrum (blue) and theoretical IR (brown) obtained by taking the absolute value of the aligned VCD spectrum. (Middle right): Aligned theoretical (red, orange) and experimental VCD spectra (black) using the VSA algorithm, Boltzmann weights based on gas-phase energies. Two conformers were considered. The alignment was performed in the wavenumber range $[1000,1500] \mathrm{cm}^{-1}$. No upwards shifts were allowed. (Bottom left): QM energy $E$ of the conformers relative to the lowest-energy conformer in the gas phase (blue), optimized for the correct enantiomer (red), and optimized for the incorrect enantiomer (orange). (Bottom right): Aligned theoretical (red, orange) and experimental VCD spectra (black) using the VSA algorithm with optimized conformer weights. 

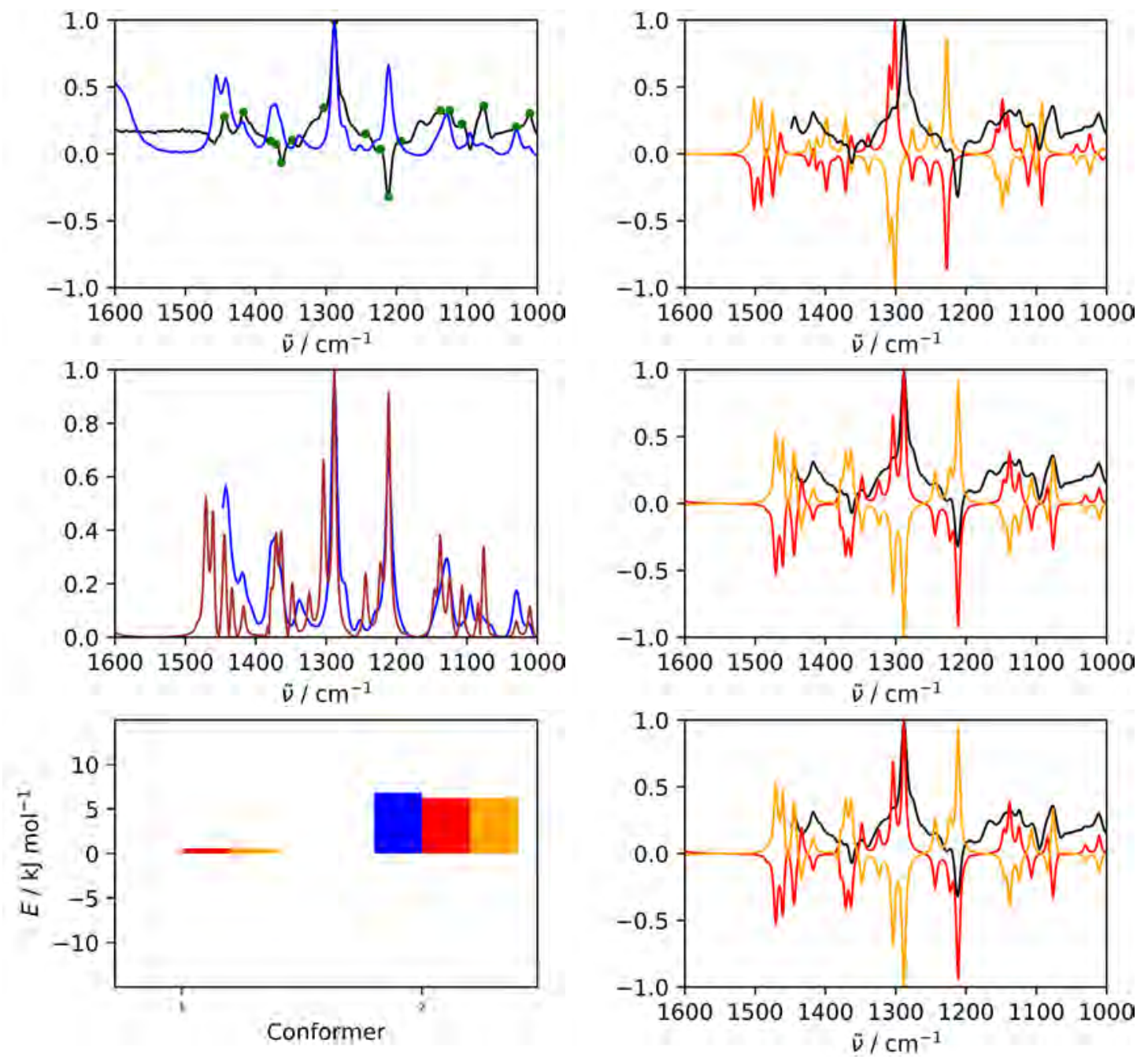

Figure S16: Compound 9, $(R)-(+)$-pulegone. (Top left): Experimental VCD spectrum (black) and IR spectrum (blue) taken from Ref. [1]. The green dots mark the peaks picked by the automatic procedure. (Top right): Unprocessed theoretical VCD spectrum of the correct enantiomer (red) and the other enantiomer (orange), as well as the experimental VCD spectrum (black). (Middle left): Experimental IR spectrum (blue) and theoretical IR (brown) obtained by taking the absolute value of the aligned VCD spectrum. (Middle right): Aligned theoretical (red, orange) and experimental VCD spectra (black) using the VSA algorithm, Boltzmann weights based on gas-phase energies. Two conformers were considered. The alignment was performed in the wavenumber range $[1000,1450] \mathrm{cm}^{-1}$. No upwards shifts were allowed. (Bottom left): QM energy $E$ of the conformers relative to the lowest-energy conformer in the gas phase (blue), optimized for the correct enantiomer (red), and optimized for the incorrect enantiomer (orange). (Bottom right): Aligned theoretical (red, orange) and experimental VCD spectra (black) using the VSA algorithm with optimized conformer weights. 

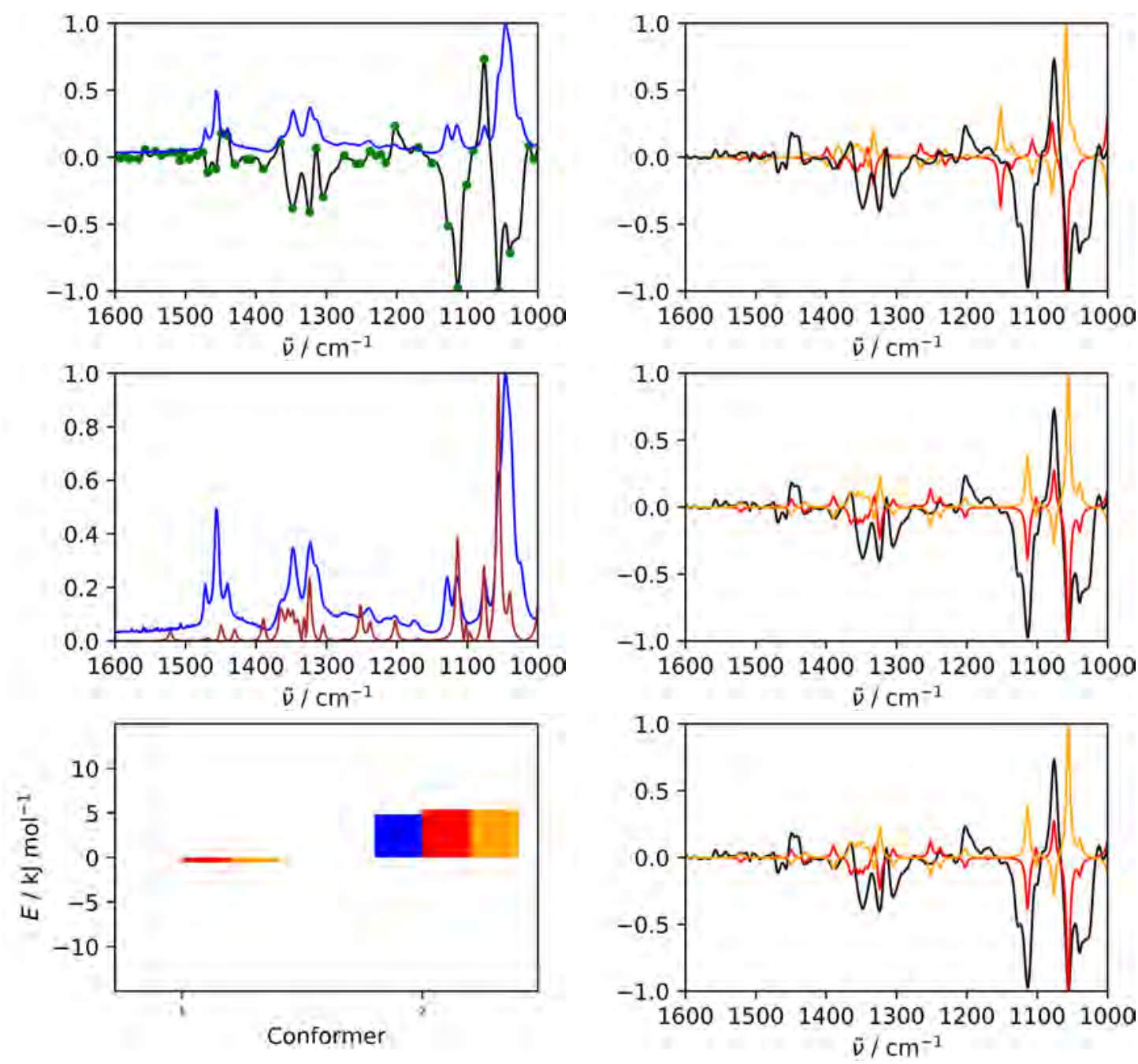

Figure S17: Compound 10, $(R)-(-)$-3-quinuclidinol. (Top left): Experimental VCD spectrum (black) and IR spectrum (blue). The green dots mark the peaks picked by the automatic procedure. (Top right): Unprocessed theoretical VCD spectrum of the correct enantiomer (red) and the other enantiomer (orange), as well as the experimental VCD spectrum (black). (Middle left): Experimental IR spectrum (blue) and theoretical IR (brown) obtained by taking the absolute value of the aligned VCD spectrum. (Middle right): Aligned theoretical (red, orange) and experimental VCD spectra (black) using the VSA algorithm, Boltzmann weights based on gas-phase energies. Two conformers were considered. The alignment was performed in the wavenumber range $[1000,1600] \mathrm{cm}^{-1}$. No upwards shifts were allowed. (Bottom left): QM energy $E$ of the conformers relative to the lowest-energy conformer in the gas phase (blue), optimized for the correct enantiomer (red), and optimized for the incorrect enantiomer (orange). (Bottom right): Aligned theoretical (red, orange) and experimental VCD spectra (black) using the VSA algorithm with optimized conformer weights. 

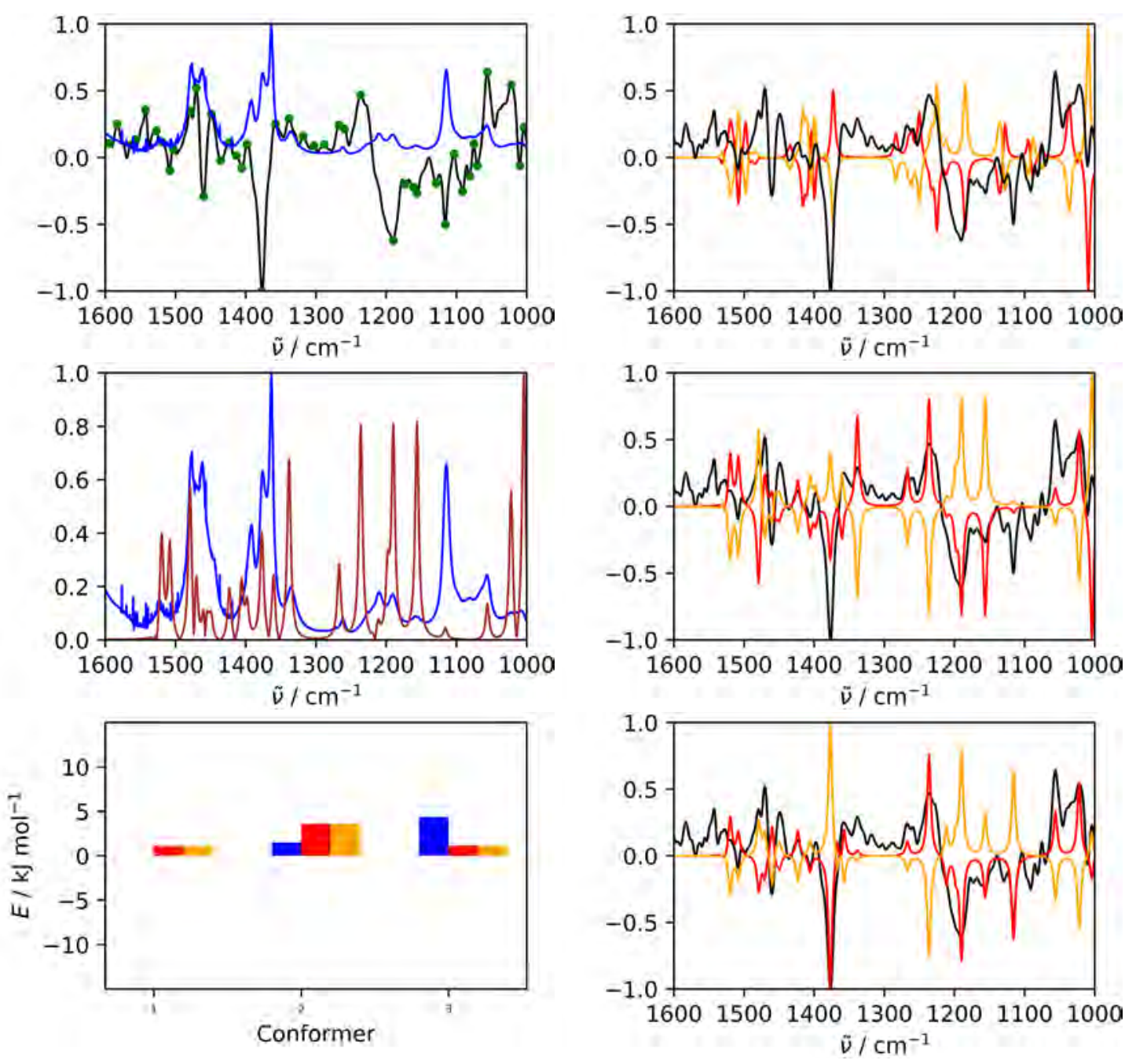

Figure S18: Compound 11, $(R)-(-)-3,3-$ dimethyl-2-butylamine. (Top left): Experimental VCD spectrum (black) and IR spectrum (blue). The green dots mark the peaks picked by the automatic procedure. (Top right): Unprocessed theoretical VCD spectrum of the correct enantiomer (red) and the other enantiomer (orange), as well as the experimental VCD spectrum (black). (Middle left): Experimental IR spectrum (blue) and theoretical IR (brown) obtained by taking the absolute value of the aligned VCD spectrum. (Middle right): Aligned theoretical (red, orange) and experimental VCD spectra (black) using the VSA algorithm, Boltzmann weights based on gas-phase energies. Three conformers were considered. The alignment was performed in the wavenumber range $[1000,1600] \mathrm{cm}^{-1}$. No upwards shifts were allowed. (Bottom left): QM energy $E$ of the conformers relative to the lowest-energy conformer in the gas phase (blue), optimized for the correct enantiomer (red), and optimized for the incorrect enantiomer (orange). (Bottom right): Aligned theoretical (red, orange) and experimental VCD spectra (black) using the VSA algorithm with optimized conformer weights. 

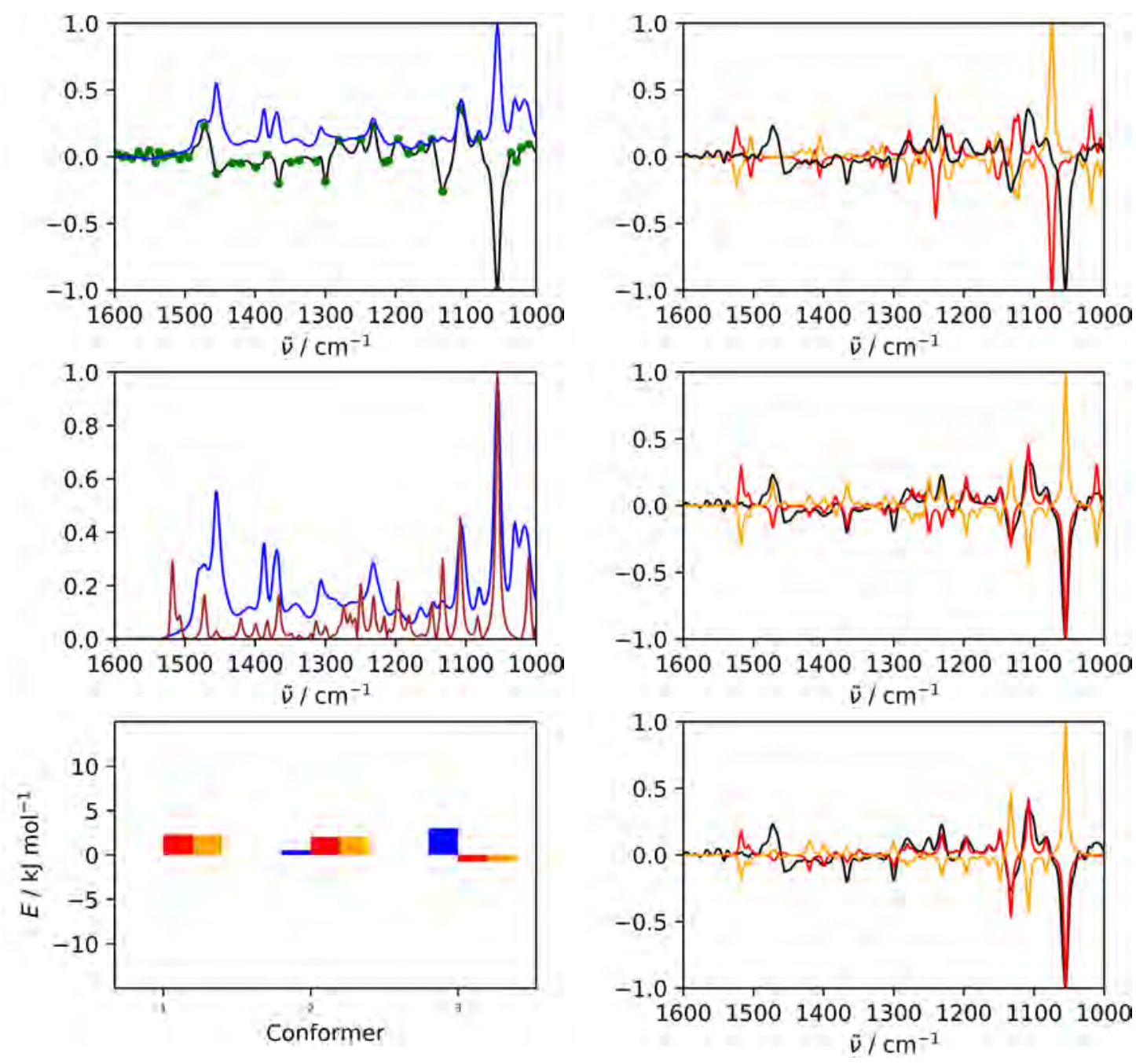

Figure S19: Compound 12, (+)-borneol. (Top left): Experimental VCD spectrum (black) and IR spectrum (blue). The green dots mark the peaks picked by the automatic procedure. (Top right): Unprocessed theoretical VCD spectrum of the correct enantiomer (red) and the other enantiomer (orange), as well as the experimental VCD spectrum (black). (Middle left): Experimental IR spectrum (blue) and theoretical IR (brown) obtained by taking the absolute value of the aligned VCD spectrum. (Middle right): Aligned theoretical (red, orange) and experimental VCD spectra (black) using the VSA algorithm, Boltzmann weights based on gas-phase energies. Three conformers were considered. The alignment was performed in the wavenumber range $[1000,1600] \mathrm{cm}^{-1}$. No upwards shifts were allowed. (Bottom left): QM energy $E$ of the conformers relative to the lowest-energy conformer in the gas phase (blue), optimized for the correct enantiomer (red), and optimized for the incorrect enantiomer (orange). (Bottom right): Aligned theoretical (red, orange) and experimental VCD spectra (black) using the VSA algorithm with optimized conformer weights. 

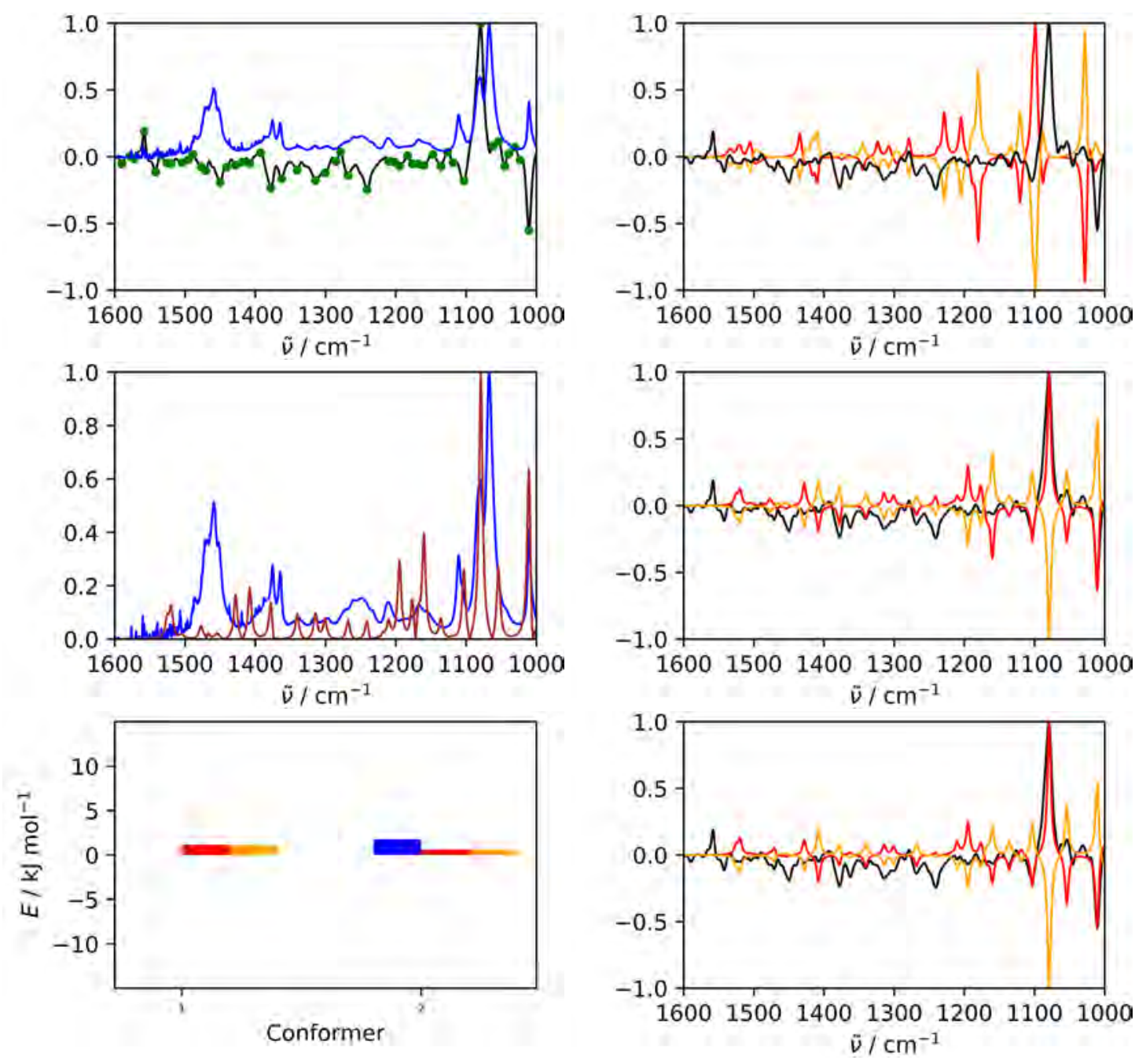

Figure S20: Compound 13, (1R)-endo-(+)-fenchylalcohol. (Top left): Experimental VCD spectrum (black) and IR spectrum (blue). The green dots mark the peaks picked by the automatic procedure. (Top right): Unprocessed theoretical VCD spectrum of the correct enantiomer (red) and the other enantiomer (orange), as well as the experimental VCD spectrum (black). (Middle left): Experimental IR spectrum (blue) and theoretical IR (brown) obtained by taking the absolute value of the aligned VCD spectrum. (Middle right): Aligned theoretical (red, orange) and experimental VCD spectra (black) using the VSA algorithm, Boltzmann weights based on gas-phase energies. Two conformers were considered. The alignment was performed in the wavenumber range $[1000,1600] \mathrm{cm}^{-1}$. No upwards shifts were allowed. (Bottom left): QM energy $E$ of the conformers relative to the lowest-energy conformer in the gas phase (blue), optimized for the correct enantiomer (red), and optimized for the incorrect enantiomer (orange). (Bottom right): Aligned theoretical (red, orange) and experimental VCD spectra (black) using the VSA algorithm with optimized conformer weights. 

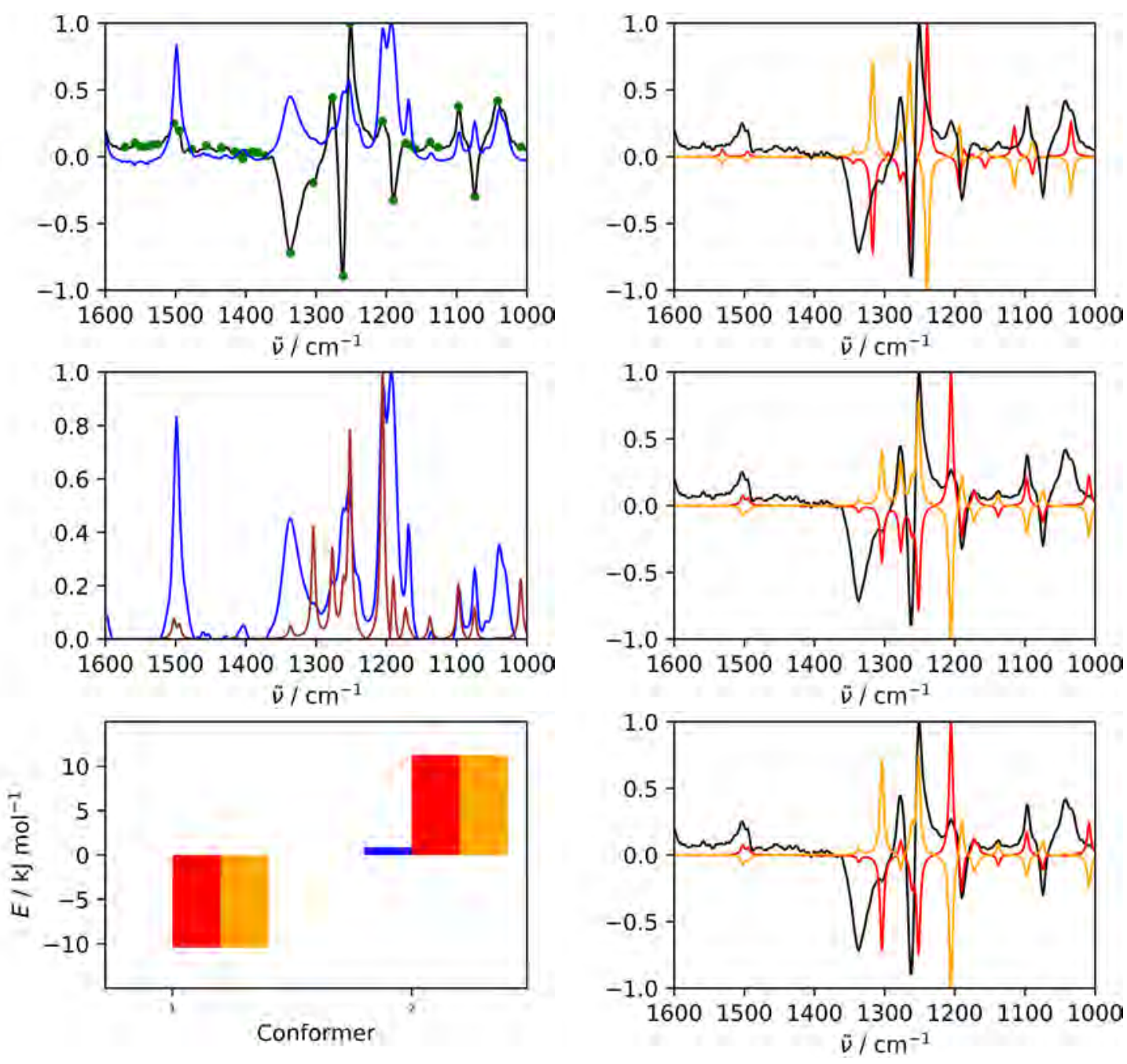

Figure S21: Compound 14, efavirenz. (Top left): Experimental VCD spectrum (black) and IR spectrum (blue) taken from Ref. [1]. The green dots mark the peaks picked by the automatic procedure. (Top right): Unprocessed theoretical VCD spectrum of the correct enantiomer (red) and the other enantiomer (orange), as well as the experimental VCD spectrum (black). (Middle left): Experimental IR spectrum (blue) and theoretical IR (brown) obtained by taking the absolute value of the aligned VCD spectrum. (Middle right): Aligned theoretical (red, orange) and experimental VCD spectra (black) using the VSA algorithm, Boltzmann weights based on gas-phase energies. Three conformers were considered. The alignment was performed in the wavenumber range $[1000,1600] \mathrm{cm}^{-1}$. No upwards shifts were allowed. (Bottom left): QM energy $E$ of the conformers relative to the lowest-energy conformer in the gas phase (blue), optimized for the correct enantiomer (red), and optimized for the incorrect enantiomer (orange). (Bottom right): Aligned theoretical (red, orange) and experimental VCD spectra (black) using the VSA algorithm with optimized conformer weights. 

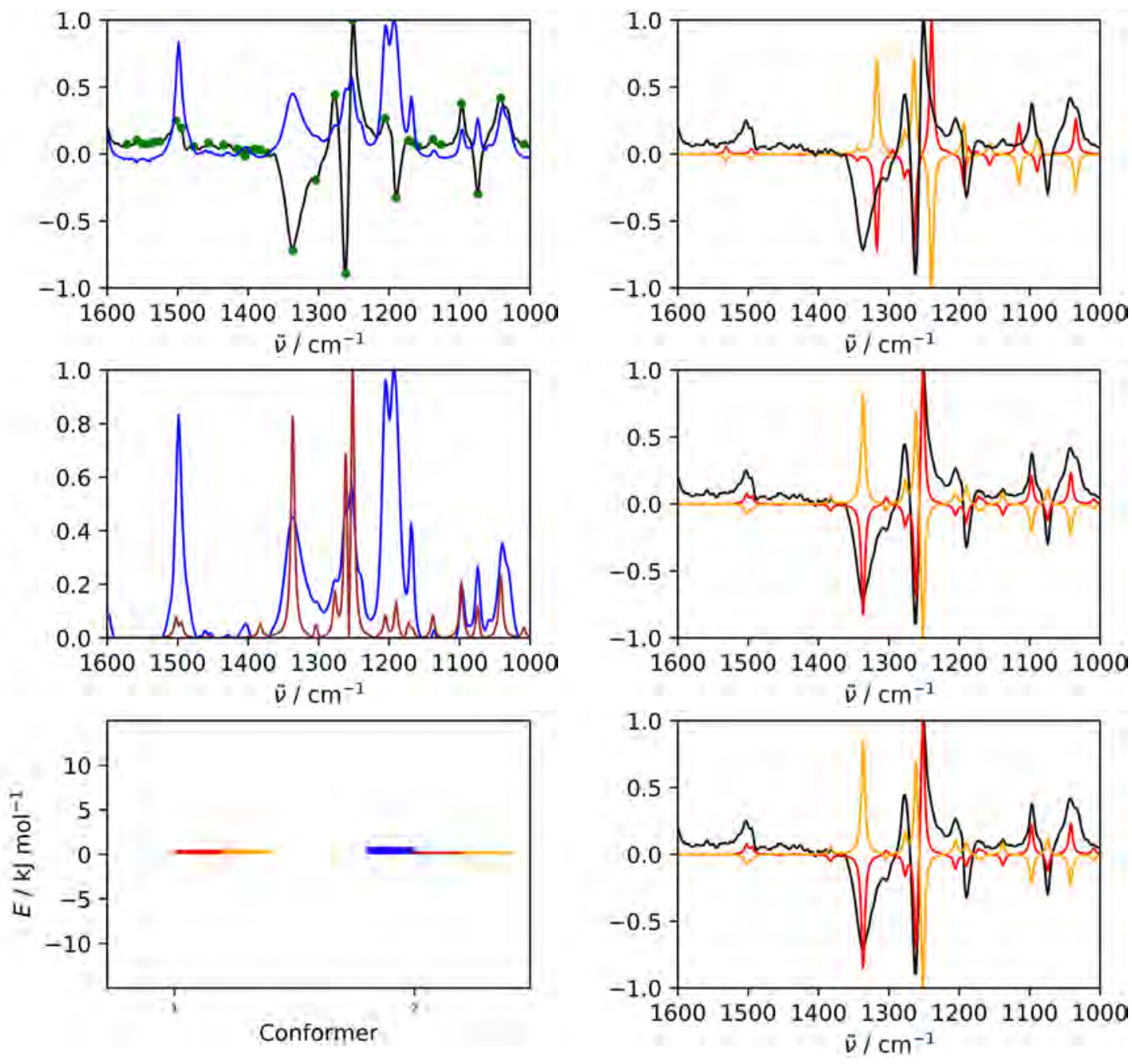

Figure S22: Compound 14, efavirenz. (Top left): Experimental VCD spectrum (black) and IR spectrum (blue) taken from Ref. [1]. The green dots mark the peaks picked by the automatic procedure. (Top right): Unprocessed theoretical VCD spectrum of the correct enantiomer (red) and the other enantiomer (orange), as well as the experimental VCD spectrum (black). (Middle left): Experimental IR spectrum (blue) and theoretical IR (brown) obtained by taking the absolute value of the aligned VCD spectrum. (Middle right): Aligned theoretical (red, orange) and experimental VCD spectra (black) using the VSA algorithm, Boltzmann weights based on gas-phase energies. Three conformers were considered. The alignment was performed in the wavenumber range $[1000,1600] \mathrm{cm}^{-1}$. Upwards shifts were allowed. (Bottom left): QM energy $E$ of the conformers relative to the lowest-energy conformer in the gas phase (blue), optimized for the correct enantiomer (red), and optimized for the incorrect enantiomer (orange). (Bottom right): Aligned theoretical (red, orange) and experimental VCD spectra (black) using the VSA algorithm with optimized conformer weights. 


\subsection{Performance with Manual Peak Selection}

Table S2: Performance of the VSA algorithm for semi-rigid molecules as measured by the Pearson coefficients $r$ for the correct (c) enantiomer, with unoptimized and optimized conformer distribution. The alignment was performed with manually picked peaks.

\begin{tabular}{ccc}
\hline Molecule & $r_{\mathrm{c}, \text { unoptimized }}$ & $r_{\mathrm{c}, \text { optimized }}$ \\
\hline \hline $\mathbf{8}$ & 0.93 & 0.93 \\
\hline $\mathbf{9}$ & 0.50 & 0.51 \\
\hline $\mathbf{1 0}$ & 0.69 & 0.74 \\
\hline $\mathbf{1 1}$ & 0.51 & 0.69 \\
\hline $\mathbf{1 2}$ & 0.78 & 0.88 \\
\hline $\mathbf{1 3}$ & 0.71 & 0.72 \\
\hline $\mathbf{1 4}$ & -0.08 & -0.12 \\
\hline
\end{tabular}



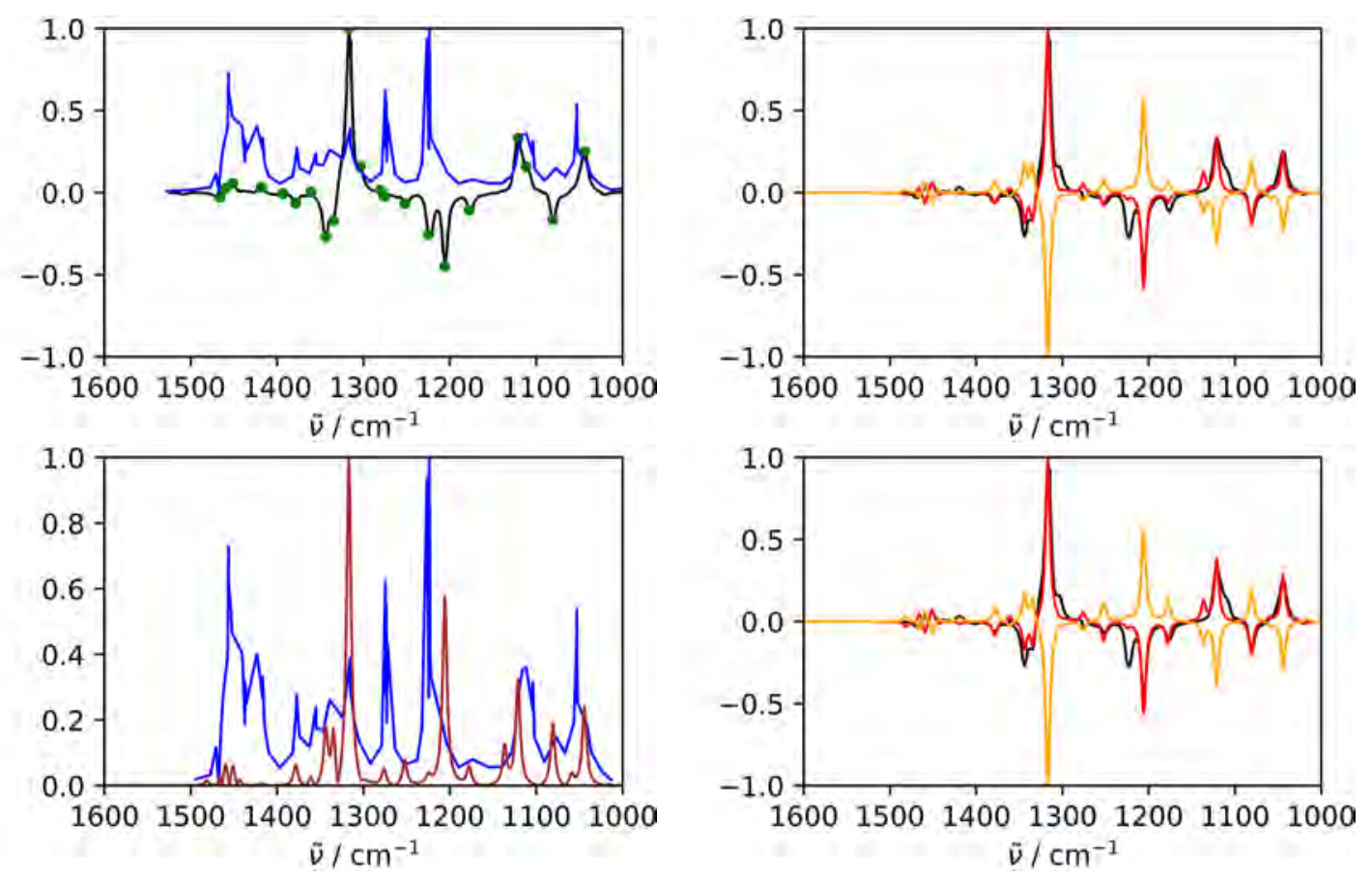

Figure S23: Compound 8, $(R)-(+)$-3-methylcyclohexanone. (Top left): Experimental VCD spectrum (black) and IR spectrum (blue) taken from Ref. [2]. The green dots mark the peaks picked manually. (Top right): Aligned theoretical VCD spectrum of the correct enantiomer (red) and the other enantiomer (orange) and experimental VCD spectra (black) using the VSA algorithm, Boltzmann weights based on gas-phase energies. Two conformers were considered. The alignment was performed in the wavenumber range $[1000,1500] \mathrm{cm}^{-1}$. No upwards shifts were allowed. (Bottom left): Experimental IR spectrum (blue) and theoretical IR (brown) obtained by taking the absolute value of the aligned VCD spectrum. (Bottom right): Aligned theoretical (red, orange) and experimental VCD spectra (black) using the VSA algorithm with optimized conformer weights. 

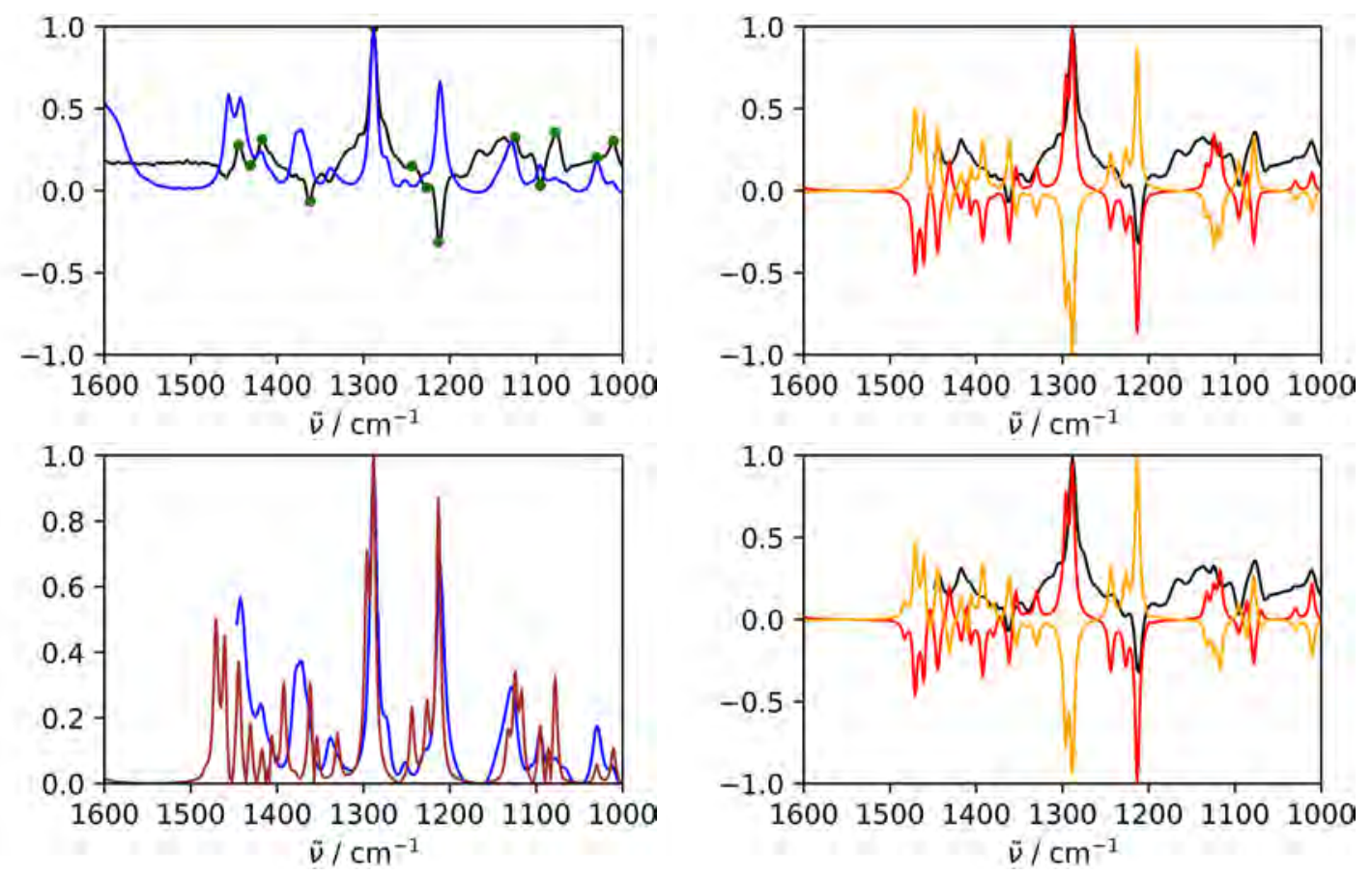

Figure S24: Compound 9, $(R)-(+)$-pulegone. (Top left): Experimental VCD spectrum (black) and IR spectrum (blue) taken from Ref. [1]. The green dots mark the peaks picked manually. (Top right): Aligned theoretical VCD spectrum of the correct enantiomer (red) and the other enantiomer (orange) and experimental VCD spectra (black) using the VSA algorithm, Boltzmann weights based on gas-phase energies. Two conformers were considered. The alignment was performed in the wavenumber range $[1000,1450] \mathrm{cm}^{-1}$. No upwards shifts were allowed. (Bottom left): Experimental IR spectrum (blue) and theoretical IR (brown) obtained by taking the absolute value of the aligned VCD spectrum. (Bottom right): Aligned theoretical (red, orange) and experimental VCD spectra (black) using the VSA algorithm with optimized conformer weights. 



Figure S25: Compound 10, $(R)-(-)$-3-quinuclidinol. (Top left): Experimental VCD spectrum (black) and IR spectrum (blue). The green dots mark the peaks picked manually. (Top right): Aligned theoretical VCD spectrum of the correct enantiomer (red) and the other enantiomer (orange) and experimental VCD spectra (black) using the VSA algorithm, Boltzmann weights based on gas-phase energies. Two conformers were considered. The alignment was performed in the wavenumber range $[1000,1600] \mathrm{cm}^{-1}$. No upwards shifts were allowed. (Bottom left): Experimental IR spectrum (blue) and theoretical IR (brown) obtained by taking the absolute value of the aligned VCD spectrum. (Bottom right): Aligned theoretical (red, orange) and experimental VCD spectra (black) using the VSA algorithm with optimized conformer weights. 

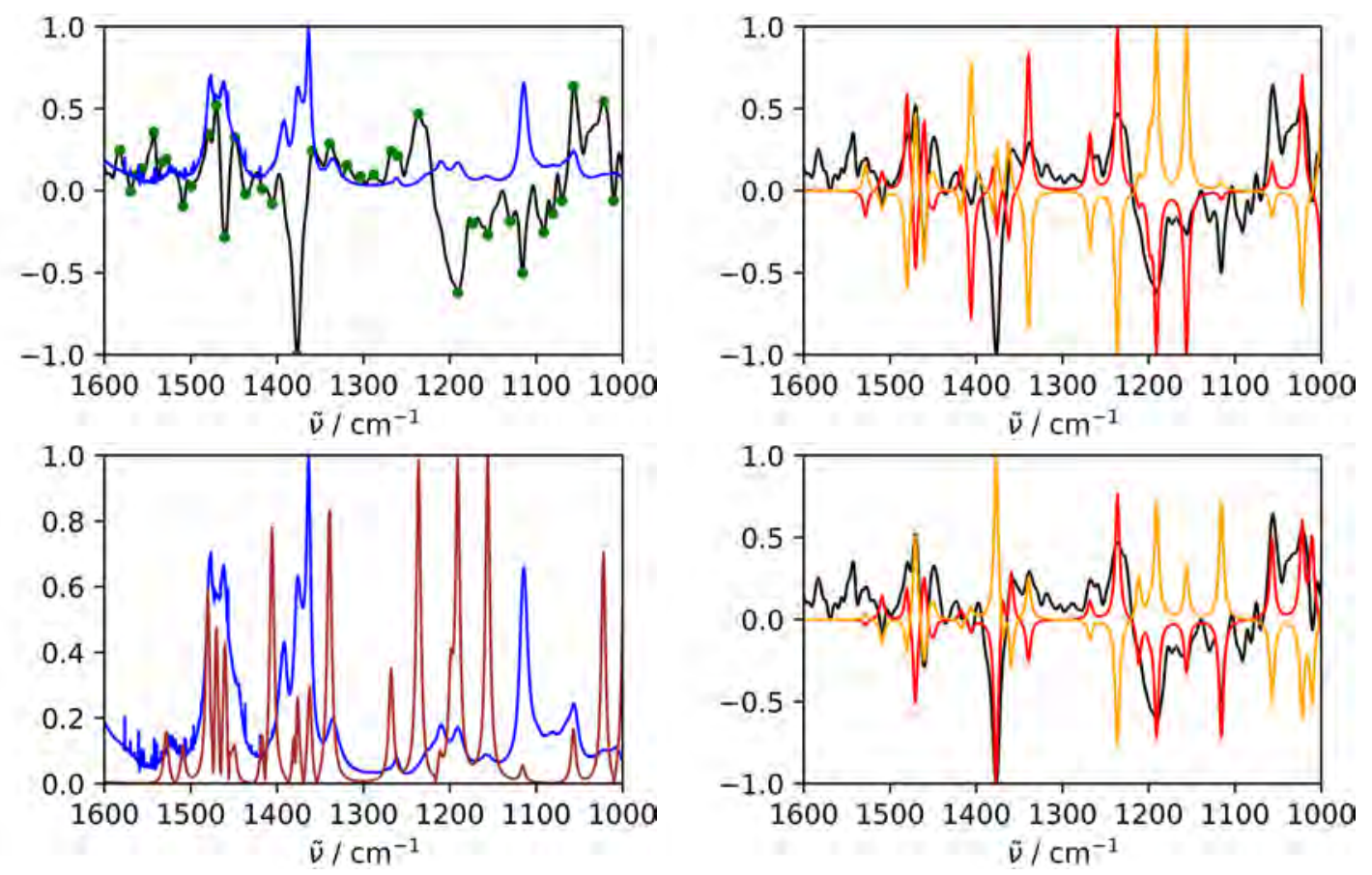

Figure S26: Compound 11, $(R)-(-)$-3,3-dimethyl-2-butylamine. (Top left): Experimental VCD spectrum (black) and IR spectrum (blue). The green dots mark the peaks picked manually. (Top right): Aligned theoretical VCD spectrum of the correct enantiomer (red) and the other enantiomer (orange) and experimental VCD spectra (black) using the VSA algorithm, Boltzmann weights based on gas-phase energies. Three conformers were considered. The alignment was performed in the wavenumber range $[1000,1600] \mathrm{cm}^{-1}$. No upwards shifts were allowed. (Bottom left): Experimental IR spectrum (blue) and theoretical IR (brown) obtained by taking the absolute value of the aligned VCD spectrum. (Bottom right): Aligned theoretical (red, orange) and experimental VCD spectra (black) using the VSA algorithm with optimized conformer weights. 

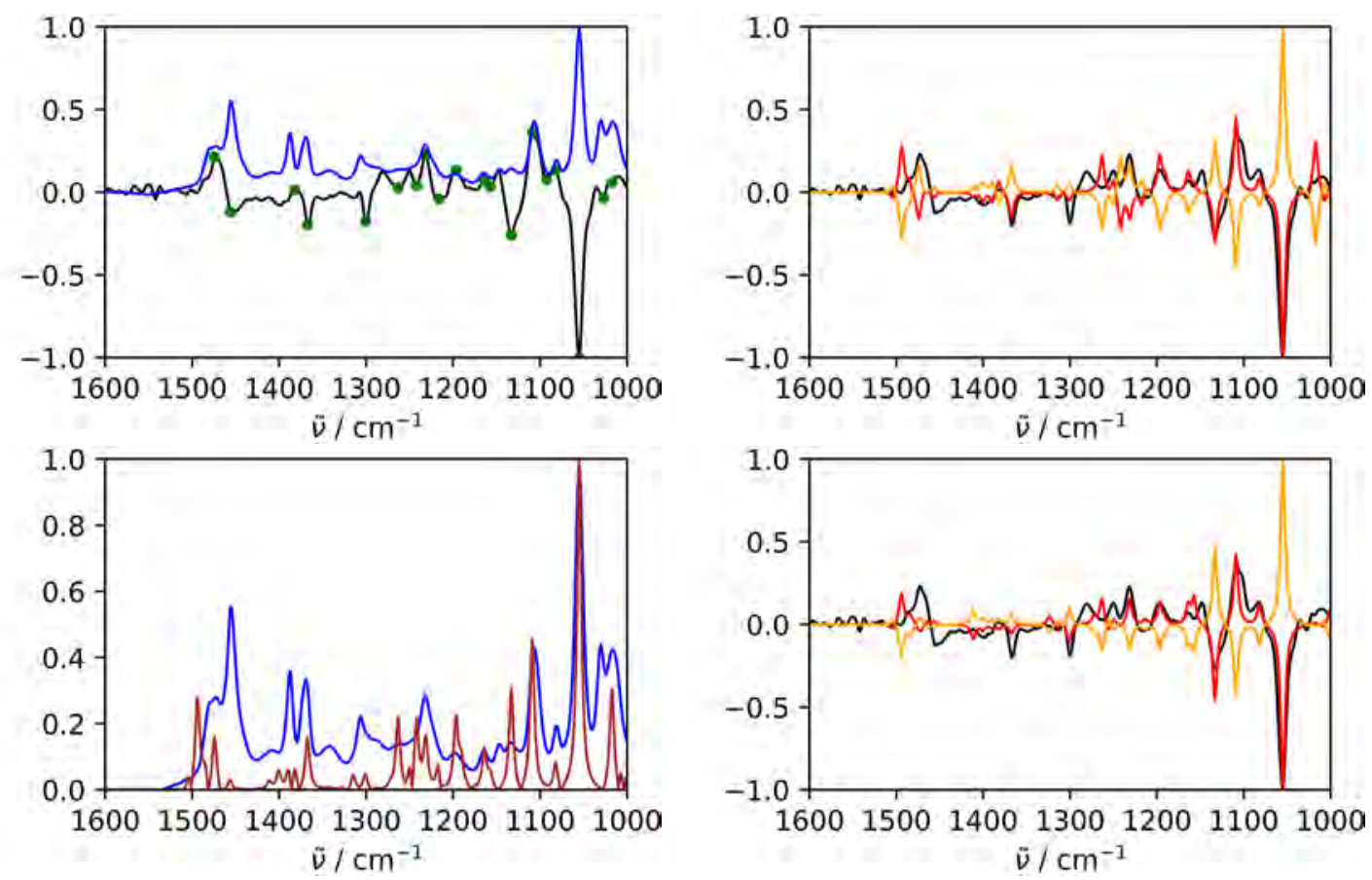

Figure S27: Compound 12, (+)-borneol. (Top left): Experimental VCD spectrum (black) and IR spectrum (blue). The green dots mark the peaks picked manually. (Top right): Aligned theoretical VCD spectrum of the correct enantiomer (red) and the other enantiomer (orange) and experimental VCD spectra (black) using the VSA algorithm, Boltzmann weights based on gas-phase energies. Three conformers were considered. The alignment was performed in the wavenumber range $[1000,1600] \mathrm{cm}^{-1}$. No upwards shifts were allowed. (Bottom left): Experimental IR spectrum (blue) and theoretical IR (brown) obtained by taking the absolute value of the aligned VCD spectrum. (Bottom right): Aligned theoretical (red, orange) and experimental VCD spectra (black) using the VSA algorithm with optimized conformer weights. 

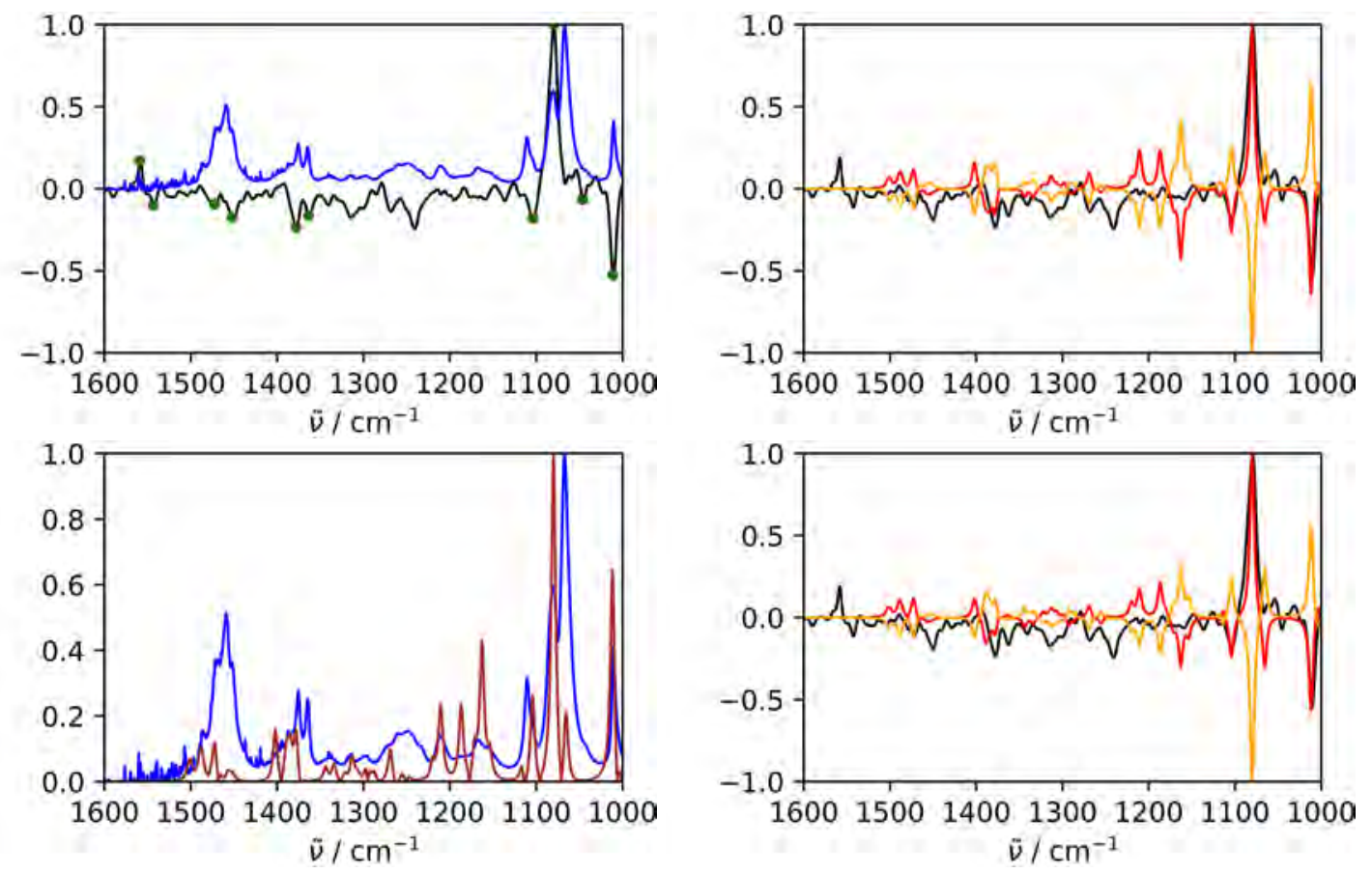

Figure S28: Compound 13, $(1 R)$-endo-(+)-fenchylalcohol. (Top left): Experimental VCD spectrum (black) and IR spectrum (blue). The green dots mark the peaks picked manually. (Top right): Aligned theoretical VCD spectrum of the correct enantiomer (red) and the other enantiomer (orange) and experimental VCD spectra (black) using the VSA algorithm, Boltzmann weights based on gas-phase energies. Two conformers were considered. The alignment was performed in the wavenumber range $[1000,1600] \mathrm{cm}^{-1}$. No upwards shifts were allowed. (Bottom left): Experimental IR spectrum (blue) and theoretical IR (brown) obtained by taking the absolute value of the aligned VCD spectrum. (Bottom right): Aligned theoretical (red, orange) and experimental VCD spectra (black) using the VSA algorithm with optimized conformer weights. 

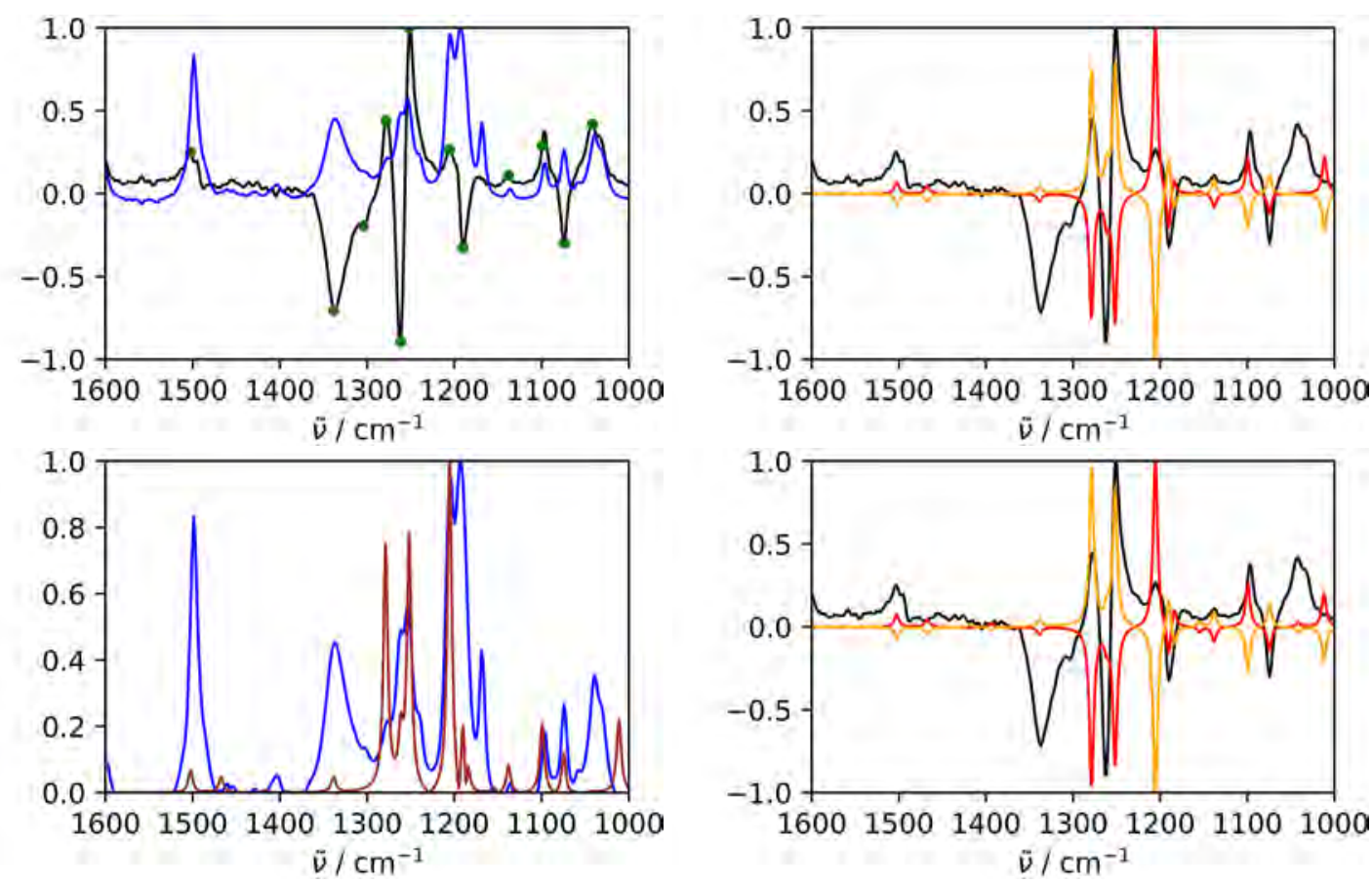

Figure S29: Compound 14, efavirenz. (Top left): Experimental VCD spectrum (black) and IR spectrum (blue) taken from Ref. [1]. The green dots mark the peaks picked manually. (Top right): Aligned theoretical VCD spectrum of the correct enantiomer (red) and the other enantiomer (orange) and experimental VCD spectra (black) using the VSA algorithm, Boltzmann weights based on gas-phase energies. Three conformers were considered. The alignment was performed in the wavenumber range $[1000,1600] \mathrm{cm}^{-1}$. No upwards shifts were allowed. (Bottom left): Experimental IR spectrum (blue) and theoretical IR (brown) obtained by taking the absolute value of the aligned VCD spectrum. (Bottom right): Aligned theoretical (red, orange) and experimental VCD spectra (black) using the VSA algorithm with optimized conformer weights. 


\section{Conformationally Flexible Molecules - All Conformers}

\subsection{Performance with Automatic Peak Selection, No Upwards Shifts Allowed}


Figure S30: Compound 15, ibuprofen. (Top left): Experimental VCD spectrum (black) and IR spectrum (blue) taken from Ref. [1]. The green dots mark the peaks picked by the automatic procedure. (Top right): Unprocessed theoretical VCD spectrum of the correct enantiomer (red) and the other enantiomer (orange), as well as the experimental VCD spectrum (black). (Middle left): Experimental IR spectrum (blue) and theoretical IR (brown) obtained by taking the absolute value of the aligned VCD spectrum. (Middle right): Aligned theoretical (red, orange) and experimental VCD spectra (black) using the VSA algorithm, Boltzmann weights based on gas-phase energies. 12 conformers were considered. The alignment was performed in the wavenumber range $[1000,1600] \mathrm{cm}^{-1}$. (Bottom left): QM energy $E$ of the conformers relative to the lowest-energy conformer in the gas phase (blue), optimized for the correct enantiomer (red), and optimized for the incorrect enantiomer (orange). (Bottom right): Aligned theoretical (red, orange) and experimental VCD spectra (black) using the VSA algorithm with optimized conformer weights. 

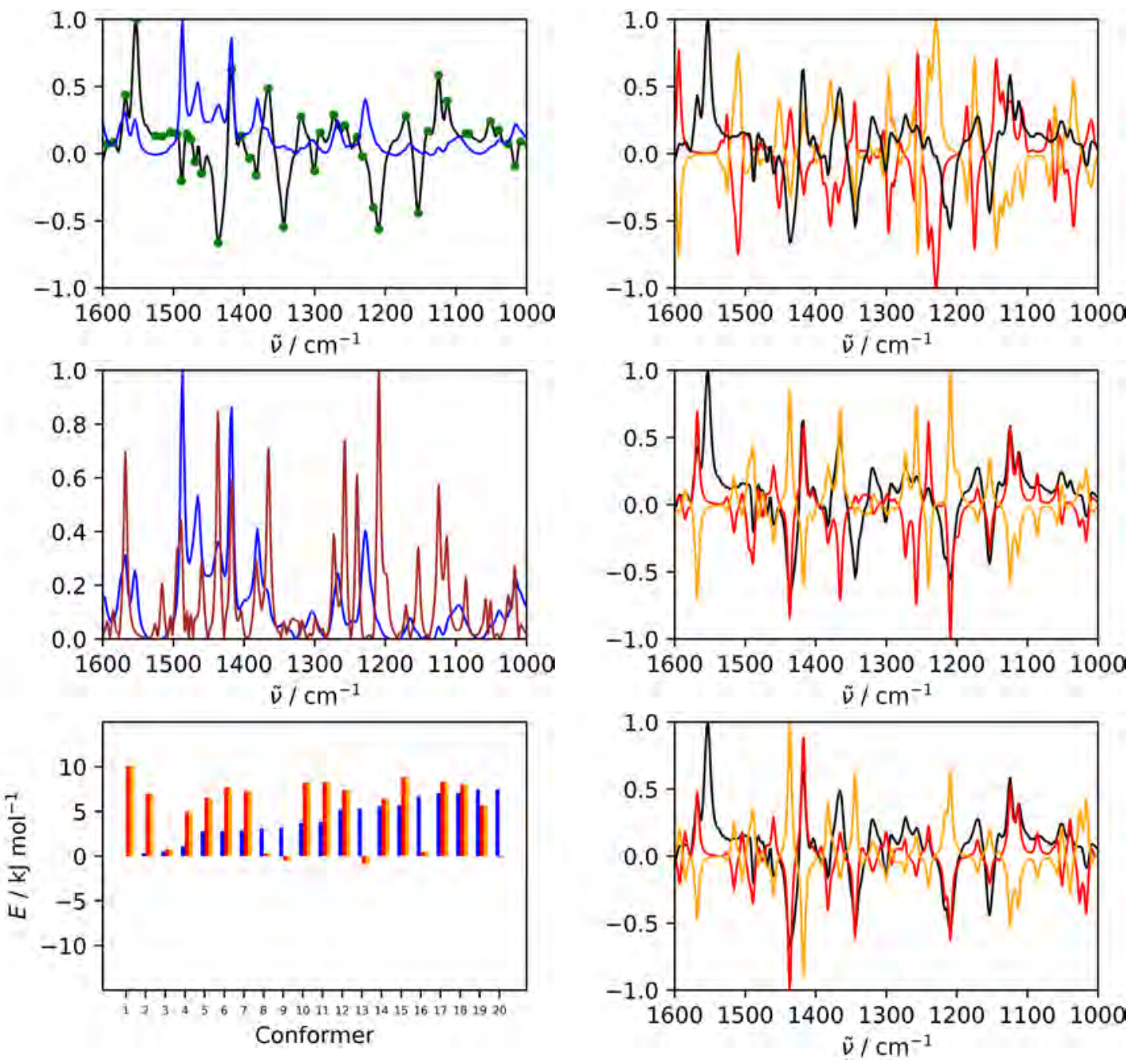

Figure S31: Compound 16, filorexant. (Top left): Experimental VCD spectrum (black) and IR spectrum (blue) taken from Ref. [1]. The green dots mark the peaks picked by the automatic procedure. (Top right): Unprocessed theoretical VCD spectrum of the correct enantiomer (red) and the other enantiomer (orange), as well as the experimental VCD spectrum (black). (Middle left): Experimental IR spectrum (blue) and theoretical IR (brown) obtained by taking the absolute value of the aligned VCD spectrum. (Middle right): Aligned theoretical (red, orange) and experimental VCD spectra (black) using the VSA algorithm, Boltzmann weights based on gas-phase energies. 126 conformers were considered. The alignment was performed in the wavenumber range $[1000,1600] \mathrm{cm}^{-1}$. (Bottom left): QM energy $E$ of the conformers relative to the lowest-energy conformer in the gas phase (blue), optimized for the correct enantiomer (red), and optimized for the incorrect enantiomer (orange). (Bottom right): Aligned theoretical (red, orange) and experimental VCD spectra (black) using the VSA algorithm with optimized conformer weights. 

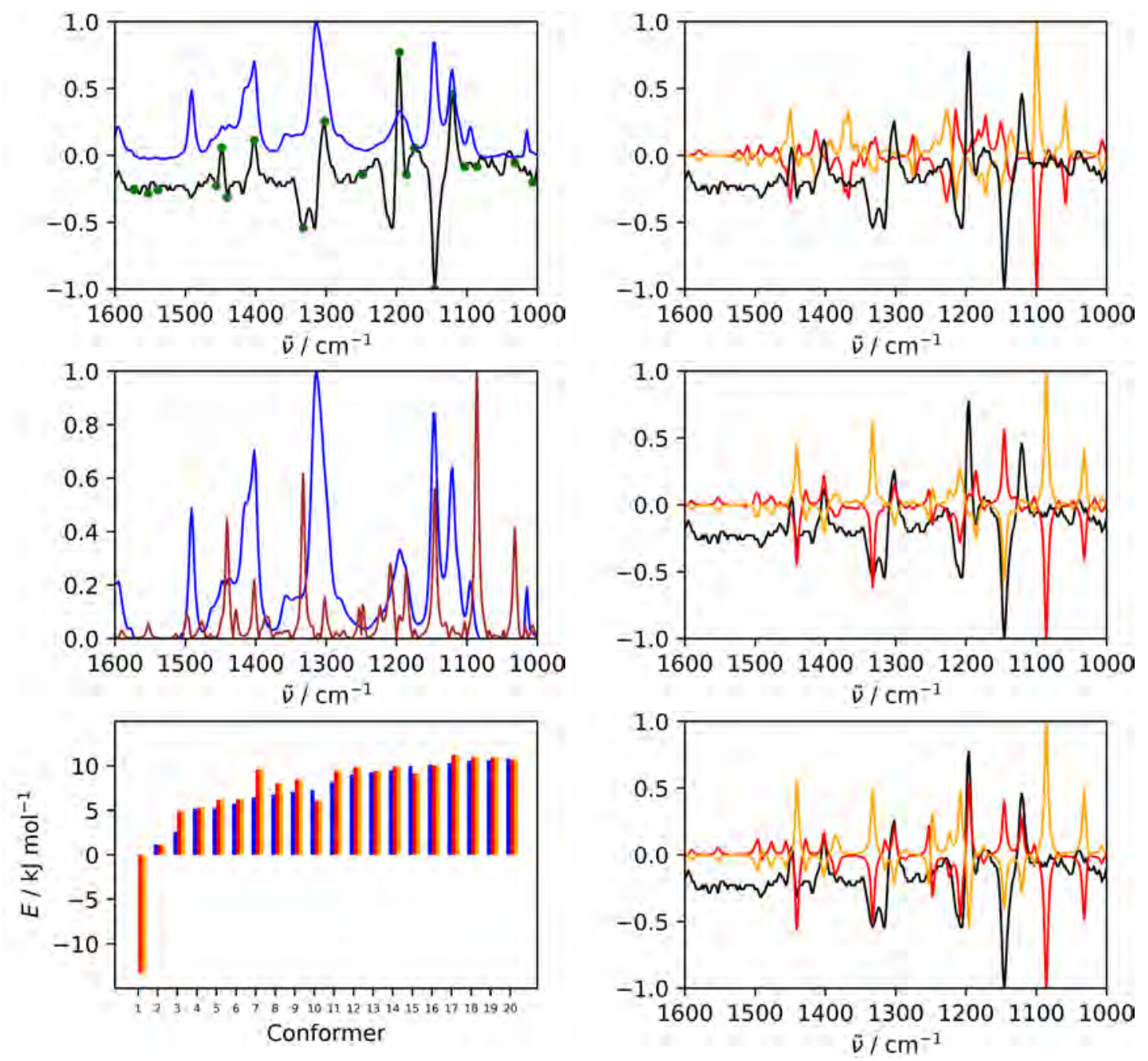

Figure S32: Compound 17, laropipirant. (Top left): Experimental VCD spectrum (black) and IR spectrum (blue) taken from Ref. [1]. The green dots mark the peaks picked by the automatic procedure. (Top right): Unprocessed theoretical VCD spectrum of the correct enantiomer (red) and the other enantiomer (orange), as well as the experimental VCD spectrum (black). (Middle left): Experimental IR spectrum (blue) and theoretical IR (brown) obtained by taking the absolute value of the aligned VCD spectrum. (Middle right): Aligned theoretical (red, orange) and experimental VCD spectra (black) using the VSA algorithm, Boltzmann weights based on gas-phase energies. 73 conformers were considered. The alignment was performed in the wavenumber range $[1000,1600] \mathrm{cm}^{-1}$. (Bottom left): QM energy $E$ of the first 20 conformers relative to the lowest-energy conformer in the gas phase (blue), optimized for the correct enantiomer (red), and optimized for the incorrect enantiomer (orange). (Bottom right): Aligned theoretical (red, orange) and experimental VCD spectra (black) using the VSA algorithm with optimized conformer weights. 

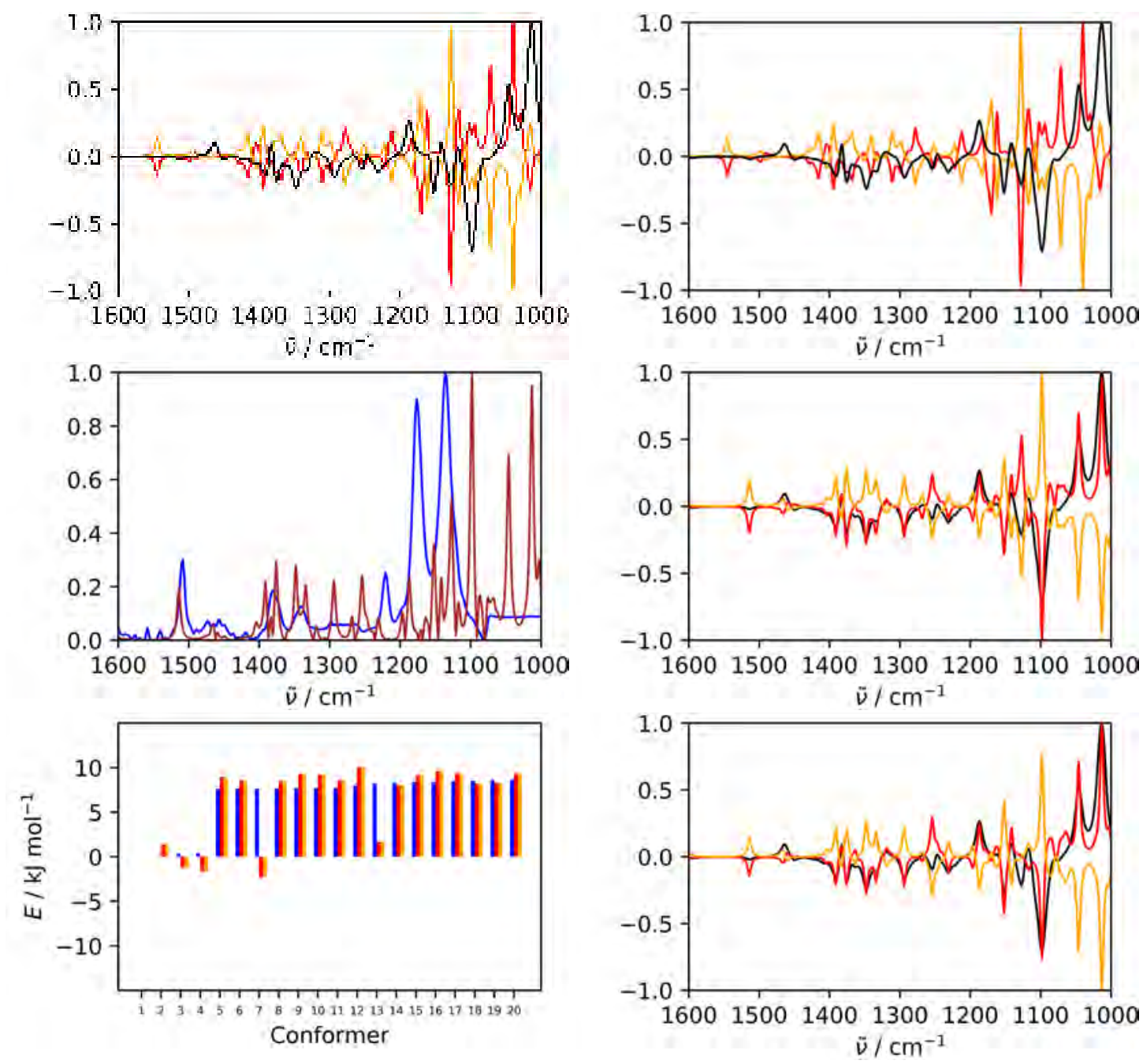

Figure S33: Compound 18, aprepitant. (Top left): Experimental VCD spectrum (black) and IR spectrum (blue) taken from Ref. [1]. The green dots mark the peaks picked by the automatic procedure. (Top right): Unprocessed theoretical VCD spectrum of the correct enantiomer (red) and the other enantiomer (orange), as well as the experimental VCD spectrum (black). (Middle left): Experimental IR spectrum (blue) and theoretical IR (brown) obtained by taking the absolute value of the aligned VCD spectrum. (Middle right): Aligned theoretical (red, orange) and experimental VCD spectra (black) using the VSA algorithm, Boltzmann weights based on gas-phase energies. 141 conformers were considered. The alignment was performed in the wavenumber range $[1000,1600] \mathrm{cm}^{-1}$. (Bottom left): QM energy $E$ of the first 20 conformers relative to the lowest-energy conformer in the gas phase (blue), optimized for the correct enantiomer (red), and optimized for the incorrect enantiomer (orange). (Bottom right): Aligned theoretical (red, orange) and experimental VCD spectra (black) using the VSA algorithm with optimized conformer weights. 

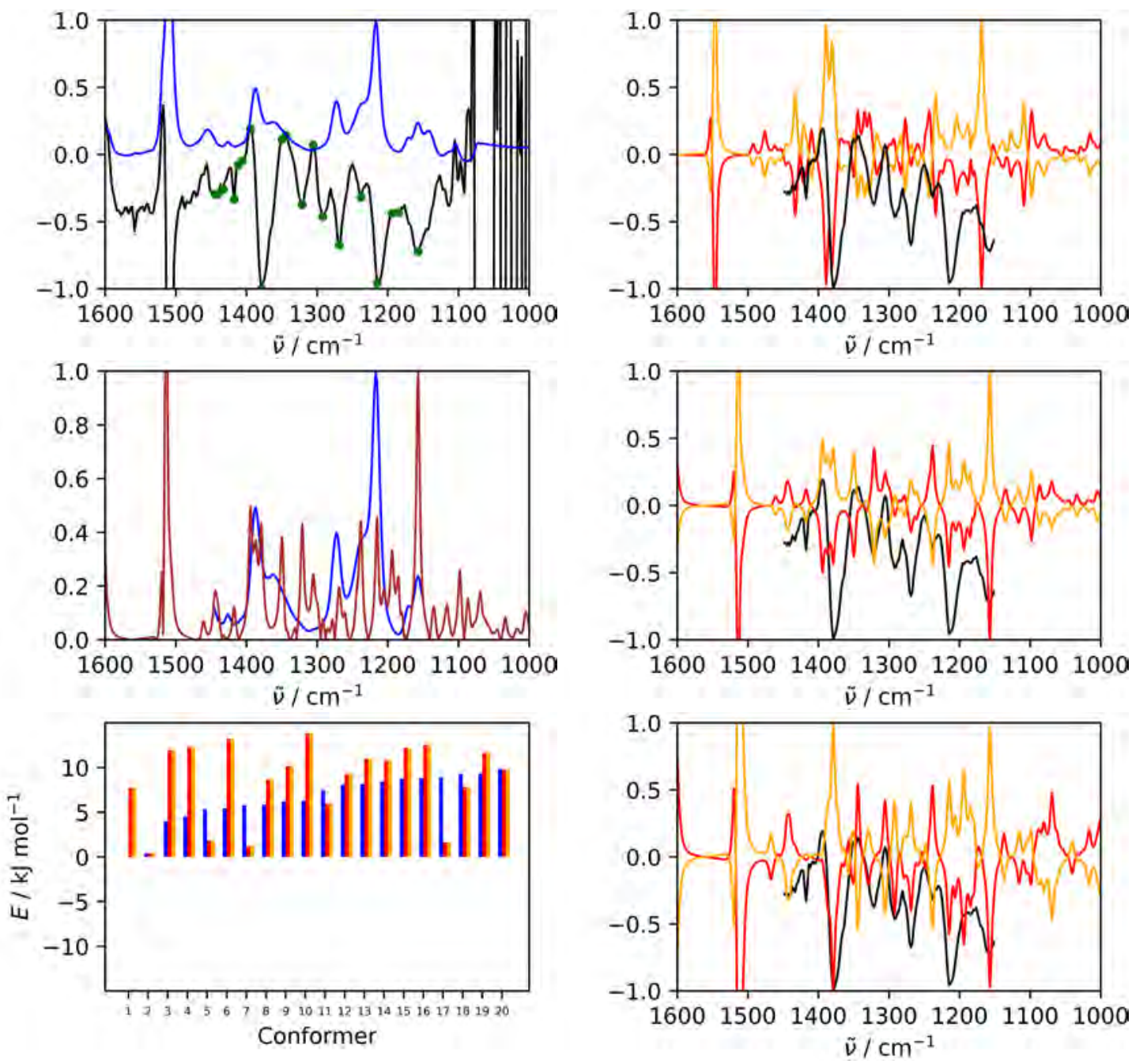

Figure S34: Compound 19, ezetimibe. (Top left): Experimental VCD spectrum (black) and IR spectrum (blue) taken from Ref. [1]. The green dots mark the peaks picked by the automatic procedure. (Top right): Unprocessed theoretical VCD spectrum of the correct enantiomer (red) and the other enantiomer (orange), as well as the experimental VCD spectrum (black). (Middle left): Experimental IR spectrum (blue) and theoretical IR (brown) obtained by taking the absolute value of the aligned VCD spectrum. (Middle right): Aligned theoretical (red, orange) and experimental VCD spectra (black) using the VSA algorithm, Boltzmann weights based on gas-phase energies. 74 conformers were considered. The alignment was performed in the wavenumber range $[1150,1450] \mathrm{cm}^{-1}$. (Bottom left): QM energy $E$ of the first 20 conformers relative to the lowest-energy conformer in the gas phase (blue), optimized for the correct enantiomer (red), and optimized for the incorrect enantiomer (orange). (Bottom right): Aligned theoretical (red, orange) and experimental VCD spectra (black) using the VSA algorithm with optimized conformer weights. 

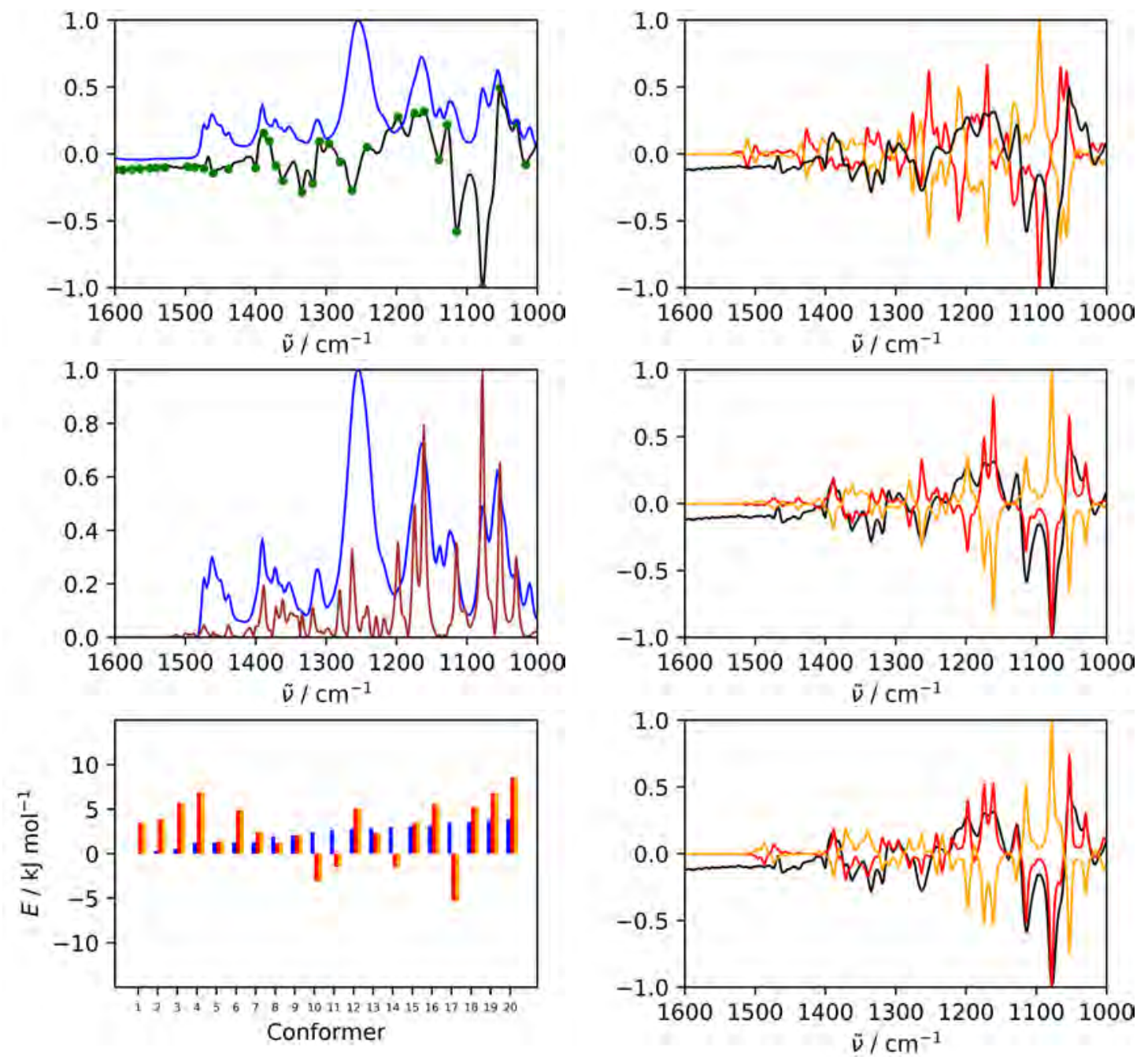

Figure S35: Compound 20, simvastatin. (Top left): Experimental VCD spectrum (black) and IR spectrum (blue) taken from Ref. [1]. The green dots mark the peaks picked by the automatic procedure. (Top right): Unprocessed theoretical VCD spectrum of the correct enantiomer (red) and the other enantiomer (orange), as well as the experimental VCD spectrum (black). (Middle left): Experimental IR spectrum (blue) and theoretical IR (brown) obtained by taking the absolute value of the aligned VCD spectrum. (Middle right): Aligned theoretical (red, orange) and experimental VCD spectra (black) using the VSA algorithm, Boltzmann weights based on gas-phase energies. 202 conformers were considered. The alignment was performed in the wavenumber range $[1000,1600] \mathrm{cm}^{-1}$. (Bottom left): QM energy $E$ of the first 20 conformers relative to the lowest-energy conformer in the gas phase (blue), optimized for the correct enantiomer (red), and optimized for the incorrect enantiomer (orange). (Bottom right): Aligned theoretical (red, orange) and experimental VCD spectra (black) using the VSA algorithm with optimized conformer weights. 

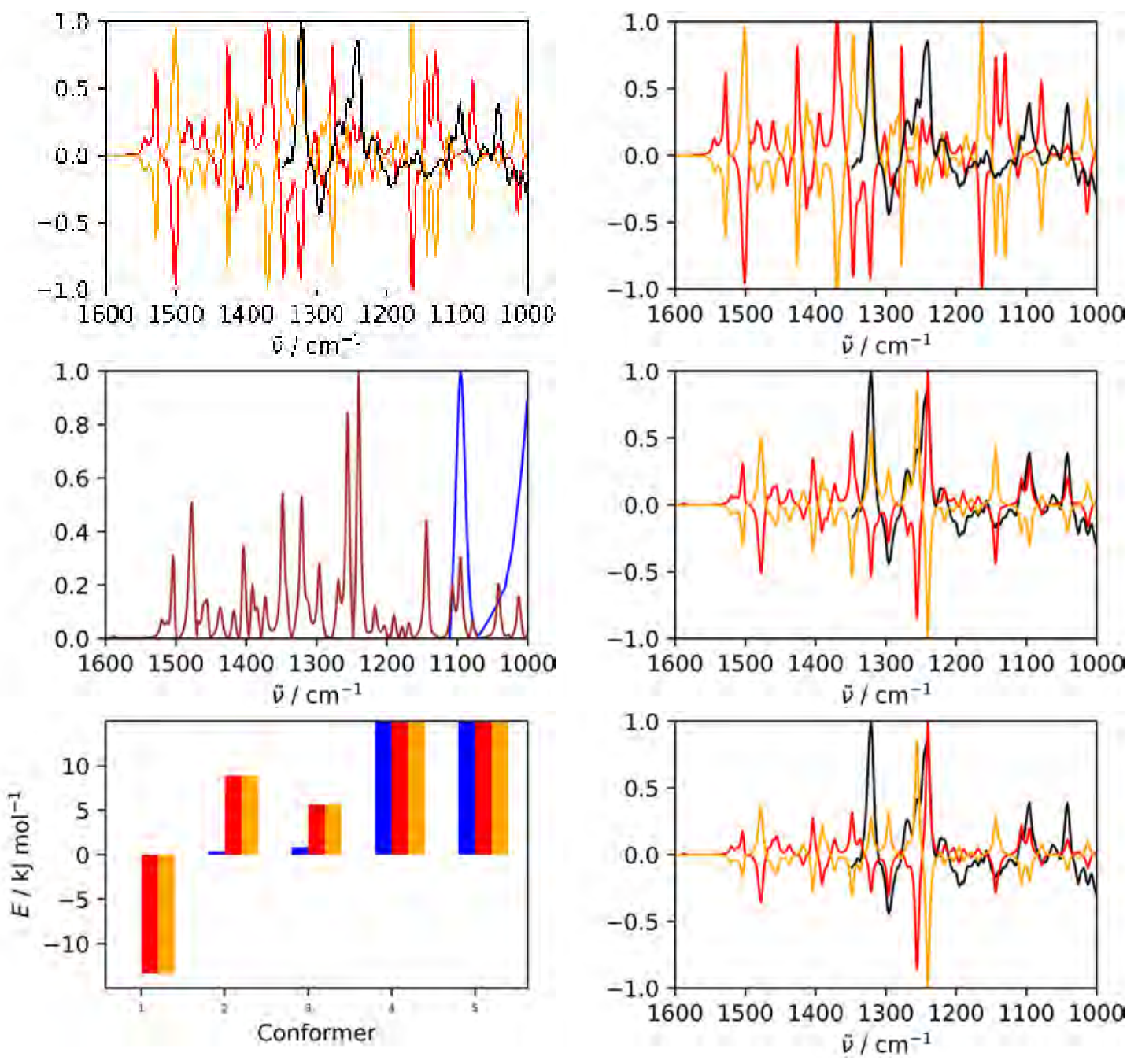

Figure S36: Compound 21, tadalafil. (Top left): Experimental VCD spectrum (black) and IR spectrum (blue). The green dots mark the peaks picked by the automatic procedure. (Top right): Unprocessed theoretical VCD spectrum of the correct enantiomer (red) and the other enantiomer (orange), as well as the experimental VCD spectrum (black). (Middle left): Experimental IR spectrum (blue) and theoretical IR (brown) obtained by taking the absolute value of the aligned VCD spectrum. (Middle right): Aligned theoretical (red, orange) and experimental VCD spectra (black) using the VSA algorithm, Boltzmann weights based on gas-phase energies. Five conformers were considered. The alignment was performed in the wavenumber range [1000, $1400 \mathrm{~cm}^{-1}$. (Bottom left): QM energy $E$ of the conformers relative to the lowest-energy conformer in the gas phase (blue), optimized for the correct enantiomer (red), and optimized for the incorrect enantiomer (orange). (Bottom right): Aligned theoretical (red, orange) and experimental VCD spectra (black) using the VSA algorithm with optimized conformer weights. 

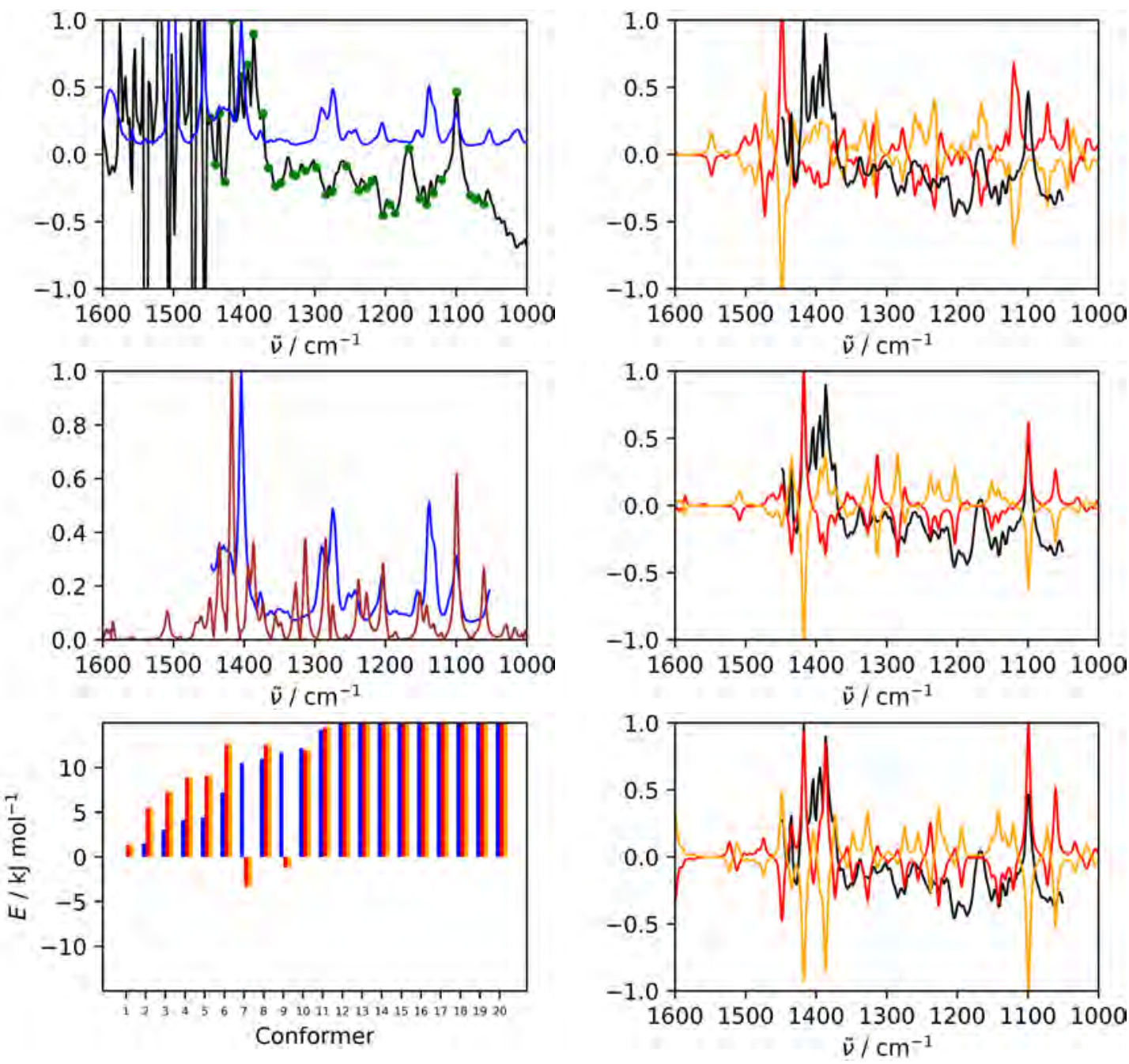

Figure S37: Compound 22, voriconazole. (Top left): Experimental VCD spectrum (black) and IR spectrum (blue). The green dots mark the peaks picked by the automatic procedure. (Top right): Unprocessed theoretical VCD spectrum of the correct enantiomer (red) and the other enantiomer (orange), as well as the experimental VCD spectrum (black). (Middle left): Experimental IR spectrum (blue) and theoretical IR (brown) obtained by taking the absolute value of the aligned VCD spectrum. (Middle right): Aligned theoretical (red, orange) and experimental VCD spectra (black) using the VSA algorithm, Boltzmann weights based on gas-phase energies. 98 conformers were considered. The alignment was performed in the wavenumber range [1050, 1450] $\mathrm{cm}^{-1}$. (Bottom left): QM energy $E$ of the first 20 conformers relative to the lowest-energy conformer in the gas phase (blue), optimized for the correct enantiomer (red), and optimized for the incorrect enantiomer (orange). (Bottom right): Aligned theoretical (red, orange) and experimental VCD spectra (black) using the VSA algorithm with optimized conformer weights. 

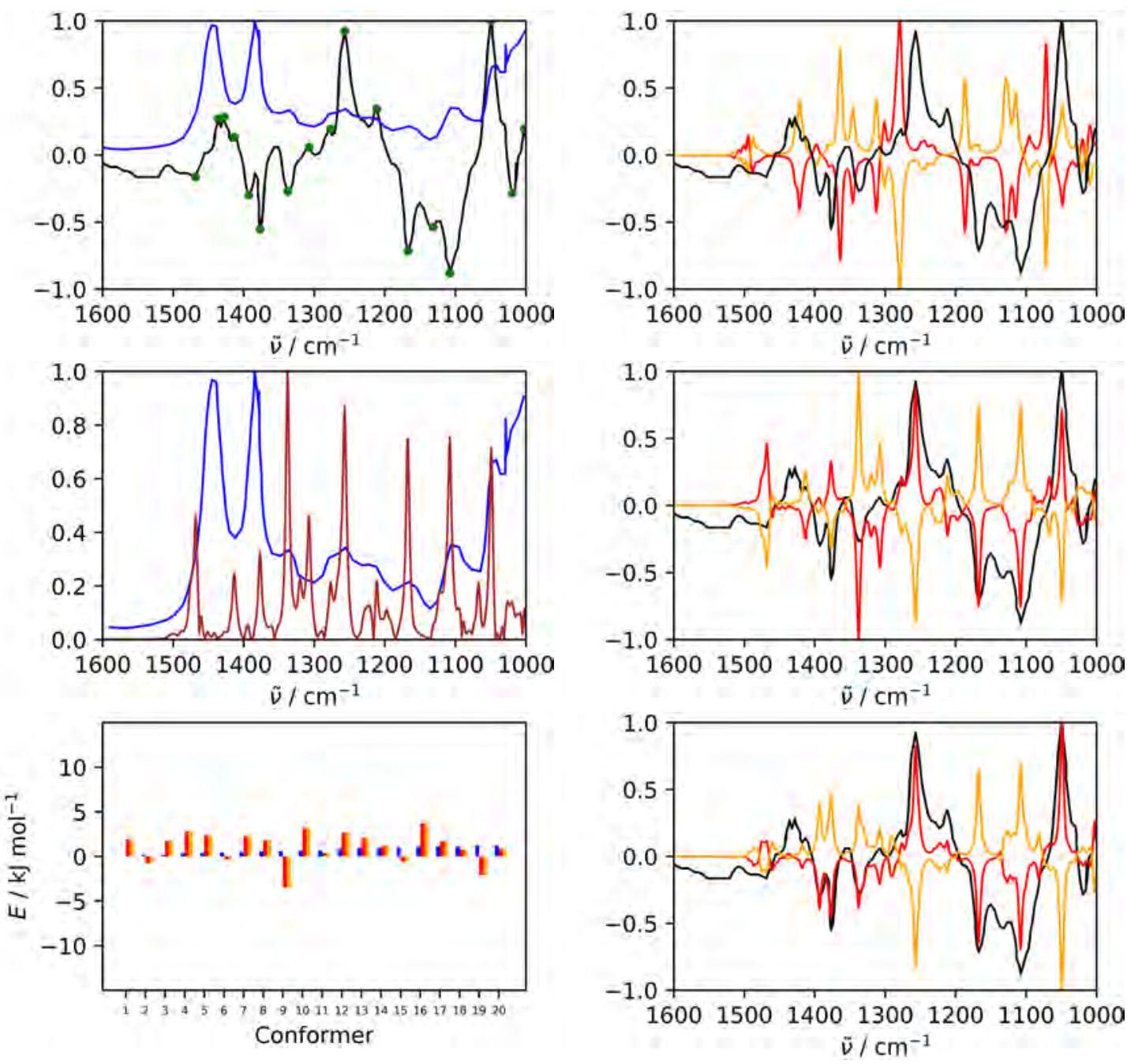

Figure S38: Compound 23, elegandiol. (Top left): Experimental VCD spectrum (black) and IR spectrum (blue) taken from Ref. [3]. The green dots mark the peaks picked by the automatic procedure. (Top right): Unprocessed theoretical VCD spectrum of the correct enantiomer (red) and the other enantiomer (orange), as well as the experimental VCD spectrum (black). (Middle left): Experimental IR spectrum (blue) and theoretical IR (brown) obtained by taking the absolute value of the aligned VCD spectrum. (Middle right): Aligned theoretical (red, orange) and experimental VCD spectra (black) using the VSA algorithm, Boltzmann weights based on gas-phase energies. 205 conformers were considered. The alignment was performed in the wavenumber range $[1000,1600] \mathrm{cm}^{-1}$. (Bottom left): QM energy $E$ of the first 20 conformers relative to the lowest-energy conformer in the gas phase (blue), optimized for the correct enantiomer (red), and optimized for the incorrect enantiomer (orange). (Bottom right): Aligned theoretical (red, orange) and experimental VCD spectra (black) using the VSA algorithm with optimized conformer weights. 

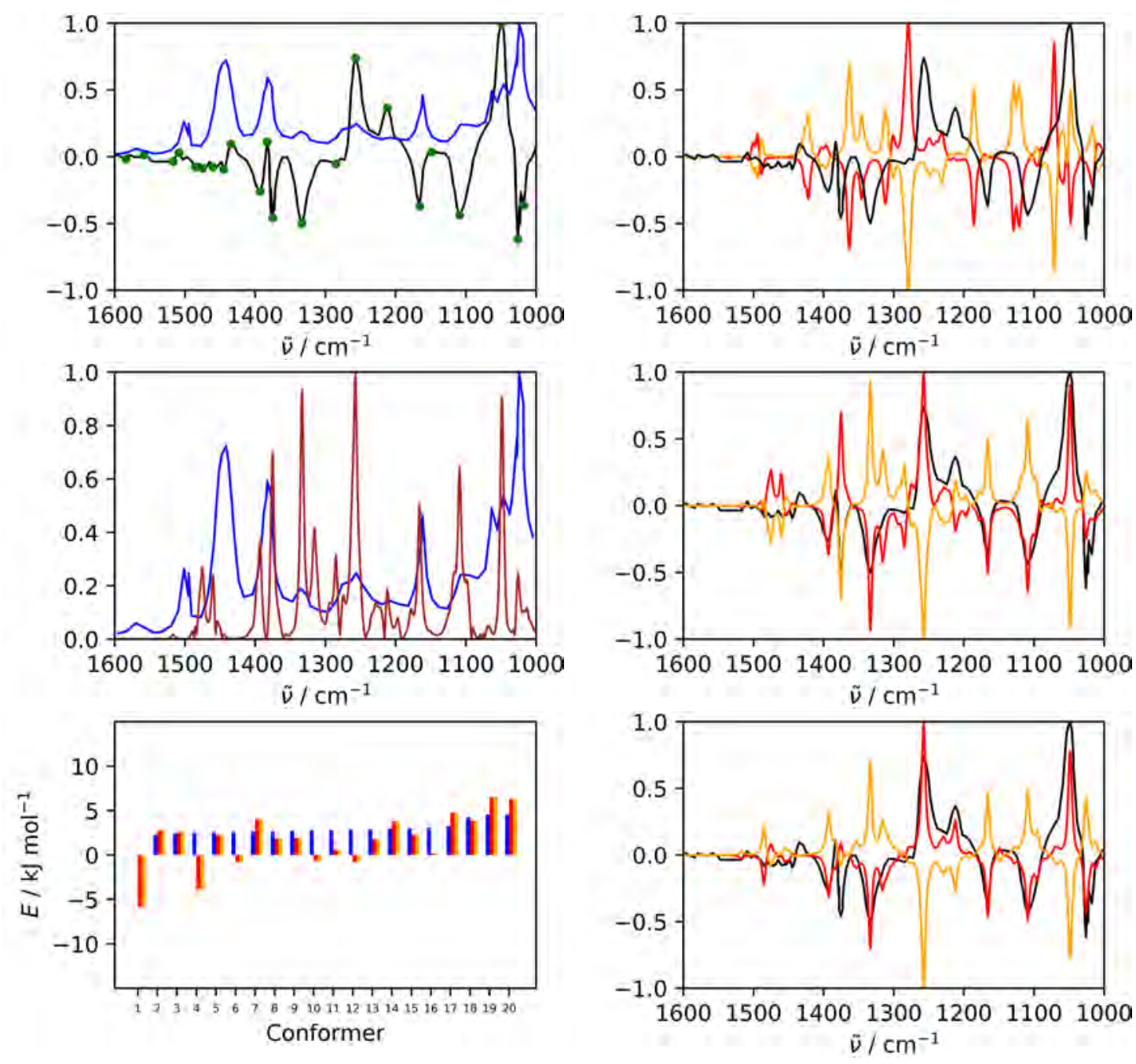

Figure S39: Compound 24, bifurcane. (Top left): Experimental VCD spectrum (black) and IR spectrum (blue) taken from Ref. [3]. The green dots mark the peaks picked by the automatic procedure. (Top right): Unprocessed theoretical VCD spectrum of the correct enantiomer (red) and the other enantiomer (orange), as well as the experimental VCD spectrum (black). (Middle left): Experimental IR spectrum (blue) and theoretical IR (brown) obtained by taking the absolute value of the aligned VCD spectrum. (Middle right): Aligned theoretical (red, orange) and experimental VCD spectra (black) using the VSA algorithm, Boltzmann weights based on gas-phase energies. 179 conformers were considered. The alignment was performed in the wavenumber range $[1000,1600] \mathrm{cm}^{-1}$. (Bottom left): QM energy $E$ of the first 20 conformers relative to the lowest-energy conformer in the gas phase (blue), optimized for the correct enantiomer (red), and optimized for the incorrect enantiomer (orange). (Bottom right): Aligned theoretical (red, orange) and experimental VCD spectra (black) using the VSA algorithm with optimized conformer weights. 

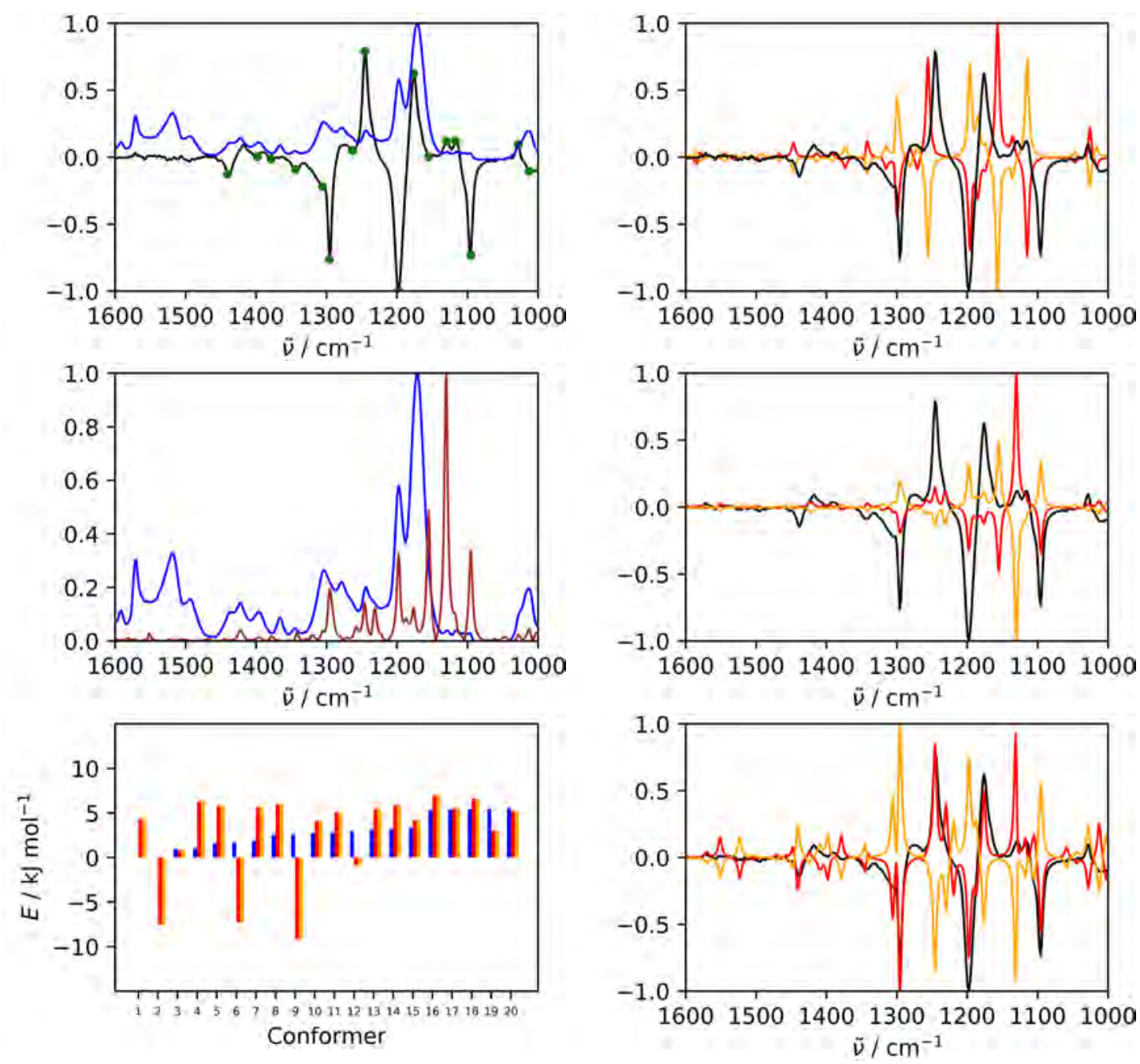

Figure S40: Compound 25, fluralaner. (Top left): Experimental VCD spectrum (black) and IR spectrum (blue) taken from Ref. [4]. The green dots mark the peaks picked by the automatic procedure. (Top right): Unprocessed theoretical VCD spectrum of the correct enantiomer (red) and the other enantiomer (orange), as well as the experimental VCD spectrum (black). (Middle left): Experimental IR spectrum (blue) and theoretical IR (brown) obtained by taking the absolute value of the aligned VCD spectrum. (Middle right): Aligned theoretical (red, orange) and experimental VCD spectra (black) using the VSA algorithm, Boltzmann weights based on gas-phase energies. 46 conformers were considered. The alignment was performed in the wavenumber range $[1000,1600] \mathrm{cm}^{-1}$. (Bottom left): QM energy $E$ of the first 20 conformers relative to the lowest-energy conformer in the gas phase (blue), optimized for the correct enantiomer (red), and optimized for the incorrect enantiomer (orange). (Bottom right): Aligned theoretical (red, orange) and experimental VCD spectra (black) using the VSA algorithm with optimized conformer weights. 


\subsection{Performance with Automatic Peak Selection, Upwards Shifts Allowed}
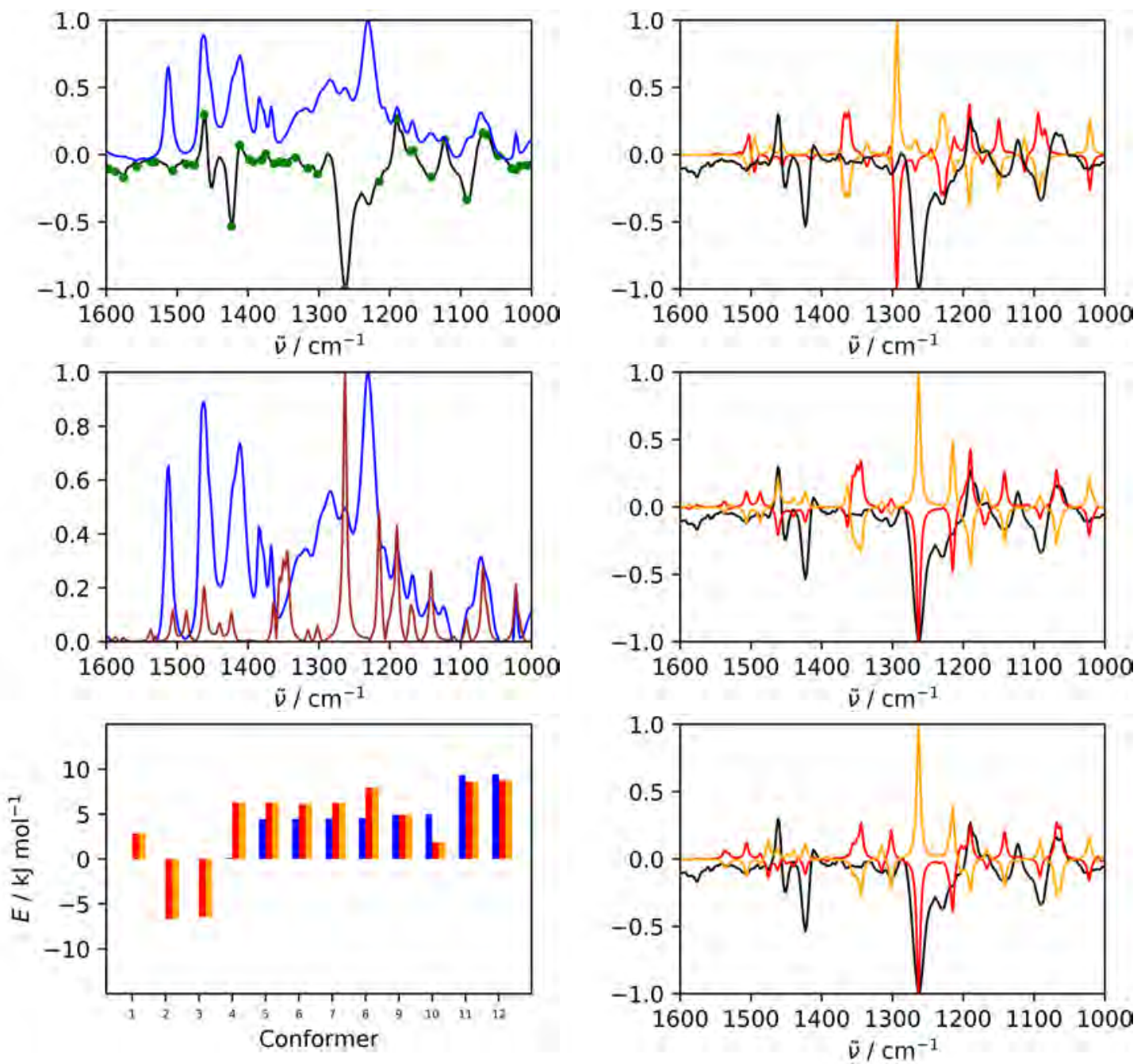

Figure S41: Compound 15, ibuprofen. (Top left): Experimental VCD spectrum (black) and IR spectrum (blue) taken from Ref. [1]. The green dots mark the peaks picked by the automatic procedure. (Top right): Unprocessed theoretical VCD spectrum of the correct enantiomer (red) and the other enantiomer (orange), as well as the experimental VCD spectrum (black). (Middle left): Experimental IR spectrum (blue) and theoretical IR (brown) obtained by taking the absolute value of the aligned VCD spectrum. (Middle right): Aligned theoretical (red, orange) and experimental VCD spectra (black) using the VSA algorithm, Boltzmann weights based on gas-phase energies. 12 conformers were considered. The alignment was performed in the wavenumber range $[1000,1600] \mathrm{cm}^{-1}$. (Bottom left): QM energy $E$ of the conformers relative to the lowest-energy conformer in the gas phase (blue), optimized for the correct enantiomer (red), and optimized for the incorrect enantiomer (orange). (Bottom right): Aligned theoretical (red, orange) and experimental VCD spectra (black) using the VSA algorithm with optimized conformer weights. 

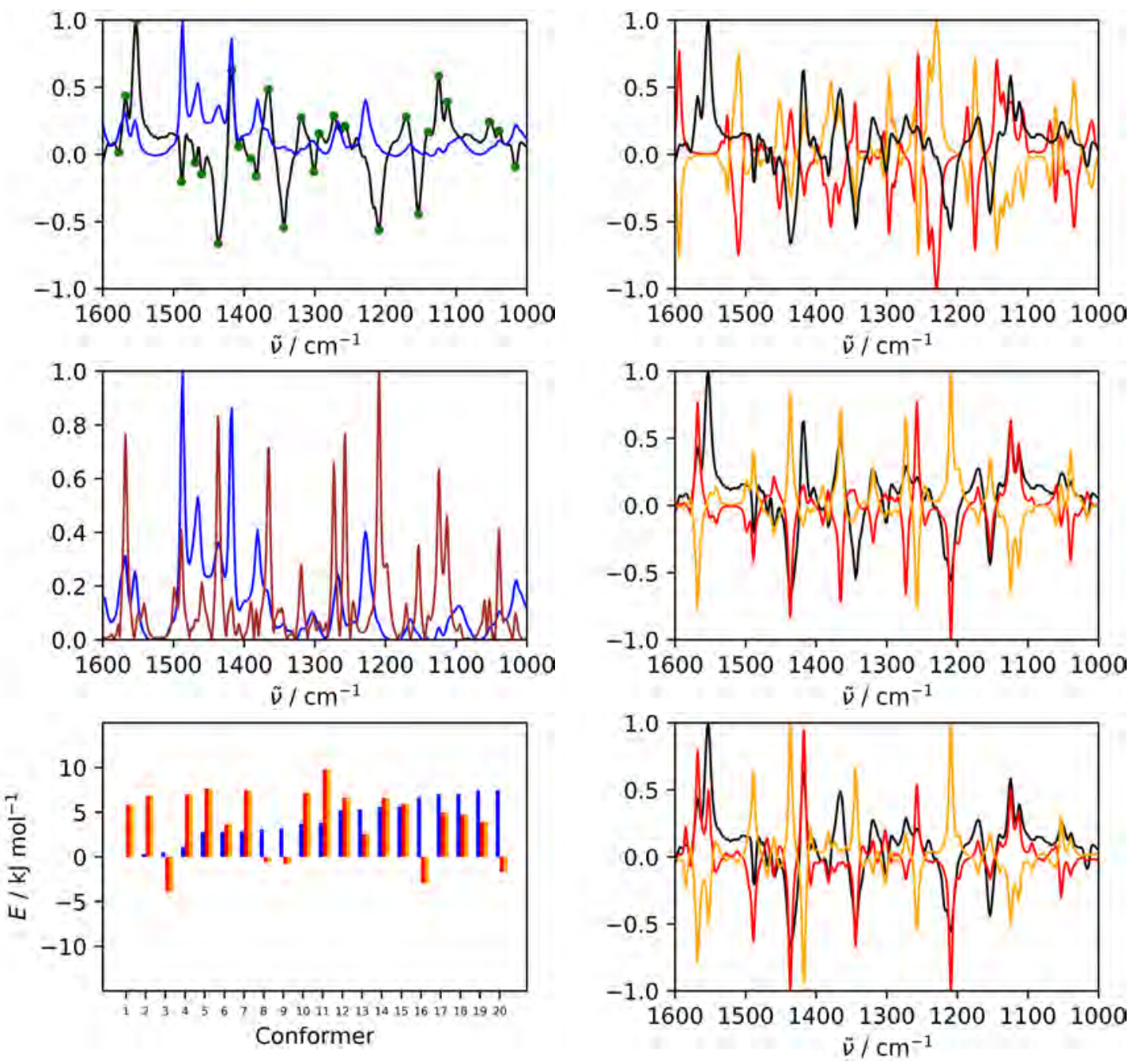

Figure S42: Compound 16, filorexant. (Top left): Experimental VCD spectrum (black) and IR spectrum (blue) taken from Ref. [1]. The green dots mark the peaks picked by the automatic procedure. (Top right): Unprocessed theoretical VCD spectrum of the correct enantiomer (red) and the other enantiomer (orange), as well as the experimental VCD spectrum (black). (Middle left): Experimental IR spectrum (blue) and theoretical IR (brown) obtained by taking the absolute value of the aligned VCD spectrum. (Middle right): Aligned theoretical (red, orange) and experimental VCD spectra (black) using the VSA algorithm, Boltzmann weights based on gas-phase energies. 126 conformers were considered. The alignment was performed in the wavenumber range $[1000,1600] \mathrm{cm}^{-1}$. (Bottom left): QM energy $E$ of the conformers relative to the lowest-energy conformer in the gas phase (blue), optimized for the correct enantiomer (red), and optimized for the incorrect enantiomer (orange). (Bottom right): Aligned theoretical (red, orange) and experimental VCD spectra (black) using the VSA algorithm with optimized conformer weights. 



Figure S43: Compound 17, laropipirant. (Top left): Experimental VCD spectrum (black) and IR spectrum (blue) taken from Ref. [1]. The green dots mark the peaks picked by the automatic procedure. (Top right): Unprocessed theoretical VCD spectrum of the correct enantiomer (red) and the other enantiomer (orange), as well as the experimental VCD spectrum (black). (Middle left): Experimental IR spectrum (blue) and theoretical IR (brown) obtained by taking the absolute value of the aligned VCD spectrum. (Middle right): Aligned theoretical (red, orange) and experimental VCD spectra (black) using the VSA algorithm, Boltzmann weights based on gas-phase energies. 73 conformers were considered. The alignment was performed in the wavenumber range $[1000,1600] \mathrm{cm}^{-1}$. (Bottom left): QM energy $E$ of the first 20 conformers relative to the lowest-energy conformer in the gas phase (blue), optimized for the correct enantiomer (red), and optimized for the incorrect enantiomer (orange). (Bottom right): Aligned theoretical (red, orange) and experimental VCD spectra (black) using the VSA algorithm with optimized conformer weights. 

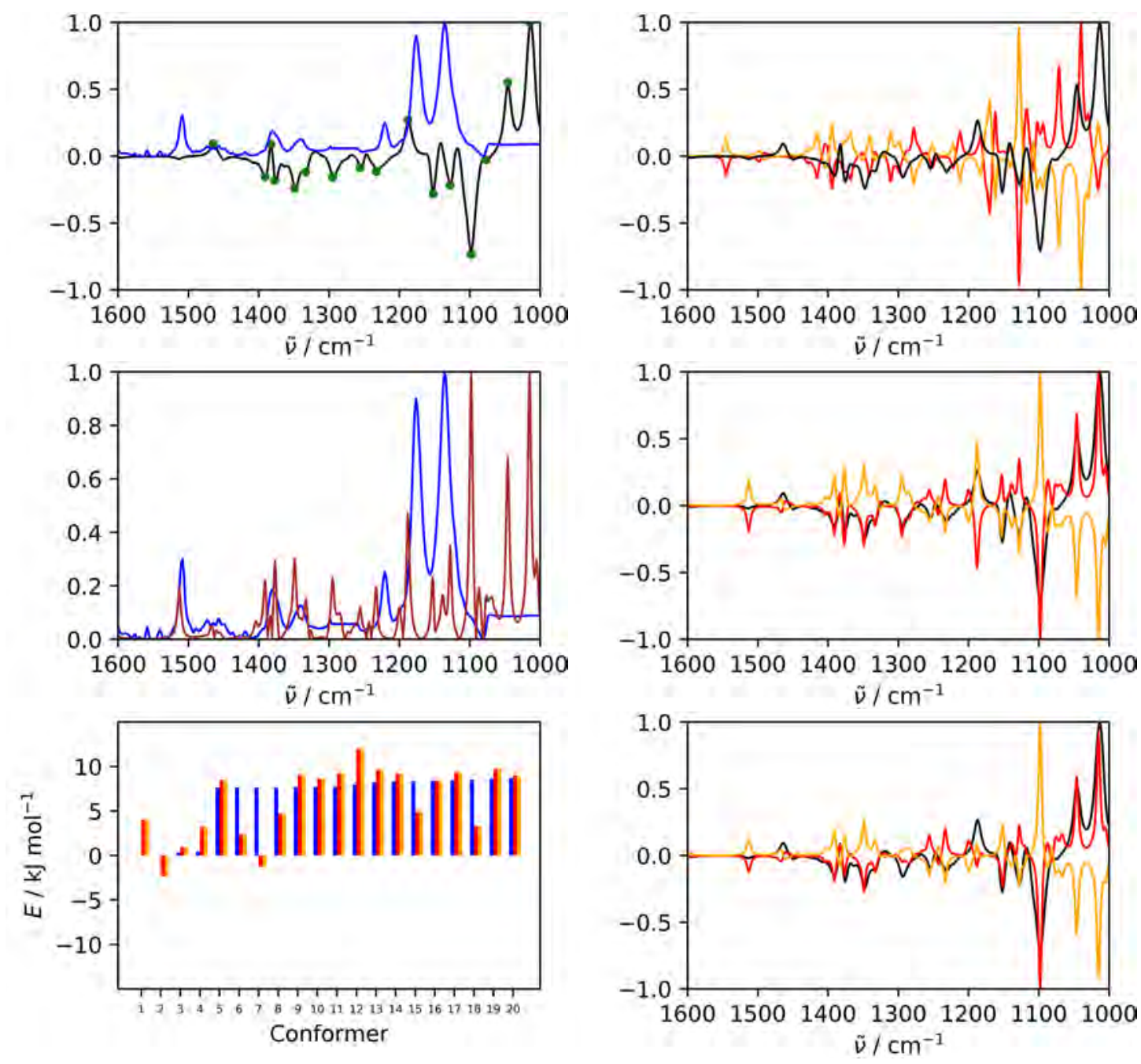

Figure S44: Compound 18, aprepitant. (Top left): Experimental VCD spectrum (black) and IR spectrum (blue) taken from Ref. [1]. The green dots mark the peaks picked by the automatic procedure. (Top right): Unprocessed theoretical VCD spectrum of the correct enantiomer (red) and the other enantiomer (orange), as well as the experimental VCD spectrum (black). (Middle left): Experimental IR spectrum (blue) and theoretical IR (brown) obtained by taking the absolute value of the aligned VCD spectrum. (Middle right): Aligned theoretical (red, orange) and experimental VCD spectra (black) using the VSA algorithm, Boltzmann weights based on gas-phase energies. 141 conformers were considered. The alignment was performed in the wavenumber range $[1000,1600] \mathrm{cm}^{-1}$. (Bottom left): QM energy $E$ of the first 20 conformers relative to the lowest-energy conformer in the gas phase (blue), optimized for the correct enantiomer (red), and optimized for the incorrect enantiomer (orange). (Bottom right): Aligned theoretical (red, orange) and experimental VCD spectra (black) using the VSA algorithm with optimized conformer weights. 

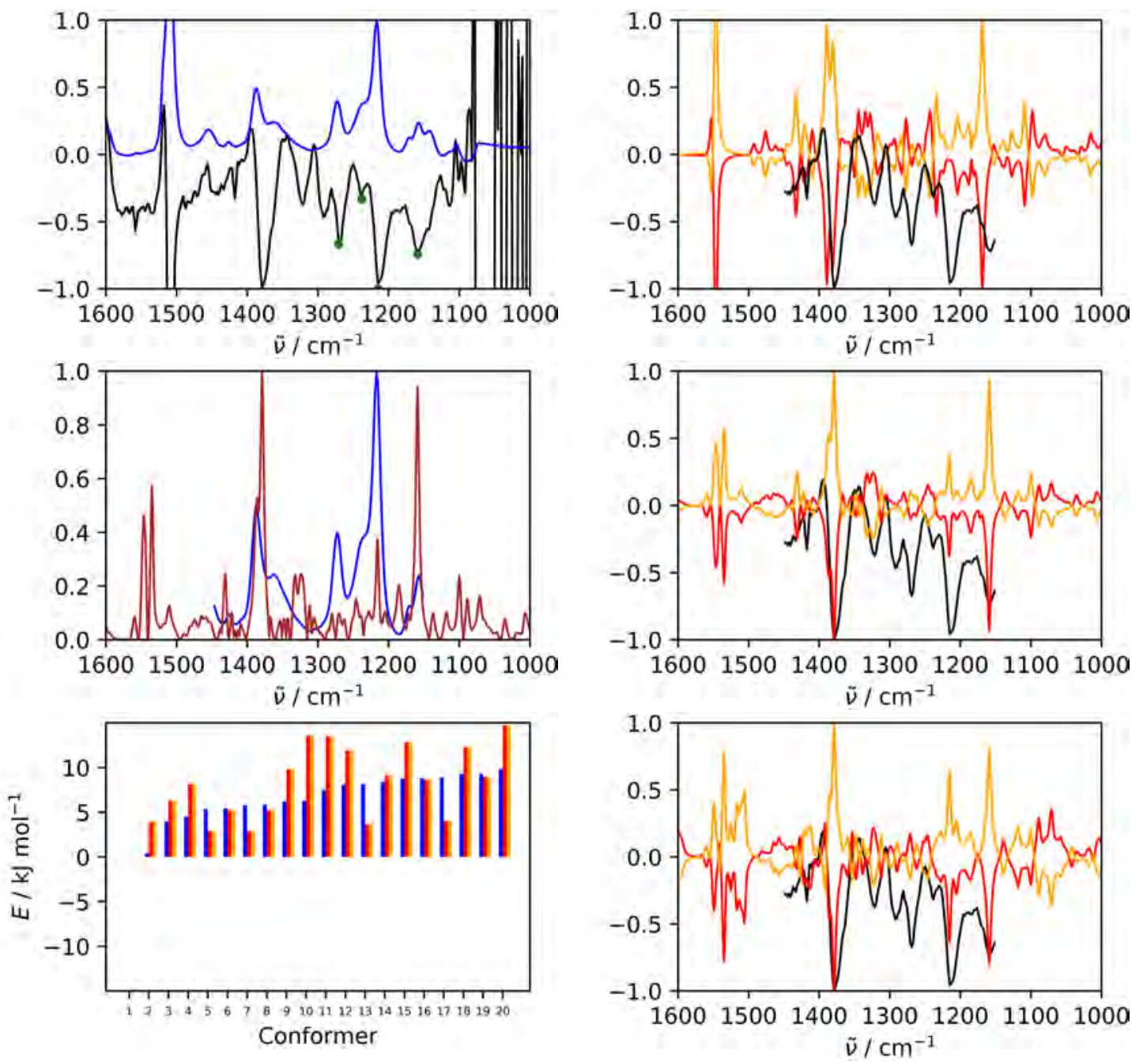

Figure S45: Compound 19, ezetimibe. (Top left): Experimental VCD spectrum (black) and IR spectrum (blue) taken from Ref. [1]. The green dots mark the peaks picked by the automatic procedure. (Top right): Unprocessed theoretical VCD spectrum of the correct enantiomer (red) and the other enantiomer (orange), as well as the experimental VCD spectrum (black). (Middle left): Experimental IR spectrum (blue) and theoretical IR (brown) obtained by taking the absolute value of the aligned VCD spectrum. (Middle right): Aligned theoretical (red, orange) and experimental VCD spectra (black) using the VSA algorithm, Boltzmann weights based on gas-phase energies. 74 conformers were considered. The alignment was performed in the wavenumber range $[1150,1450] \mathrm{cm}^{-1}$. (Bottom left): QM energy $E$ of the first 20 conformers relative to the lowest-energy conformer in the gas phase (blue), optimized for the correct enantiomer (red), and optimized for the incorrect enantiomer (orange). (Bottom right): Aligned theoretical (red, orange) and experimental VCD spectra (black) using the VSA algorithm with optimized conformer weights. 

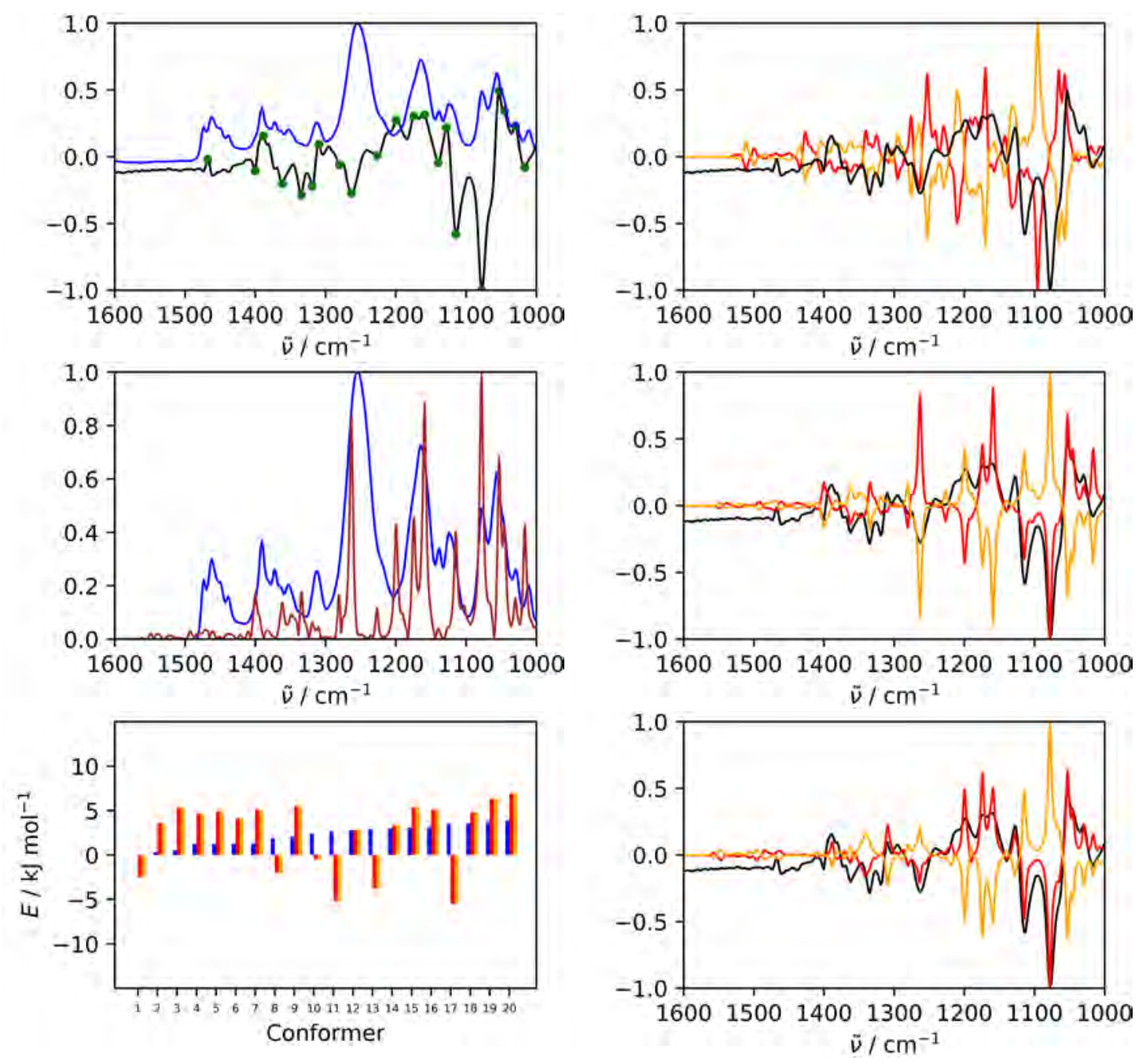

Figure S46: Compound 20, simvastatin. (Top left): Experimental VCD spectrum (black) and IR spectrum (blue) taken from Ref. [1]. The green dots mark the peaks picked by the automatic procedure. (Top right): Unprocessed theoretical VCD spectrum of the correct enantiomer (red) and the other enantiomer (orange), as well as the experimental VCD spectrum (black). (Middle left): Experimental IR spectrum (blue) and theoretical IR (brown) obtained by taking the absolute value of the aligned VCD spectrum. (Middle right): Aligned theoretical (red, orange) and experimental VCD spectra (black) using the VSA algorithm, Boltzmann weights based on gas-phase energies. 202 conformers were considered. The alignment was performed in the wavenumber range $[1000,1600] \mathrm{cm}^{-1}$. (Bottom left): QM energy $E$ of the first 20 conformers relative to the lowest-energy conformer in the gas phase (blue), optimized for the correct enantiomer (red), and optimized for the incorrect enantiomer (orange). (Bottom right): Aligned theoretical (red, orange) and experimental VCD spectra (black) using the VSA algorithm with optimized conformer weights. 

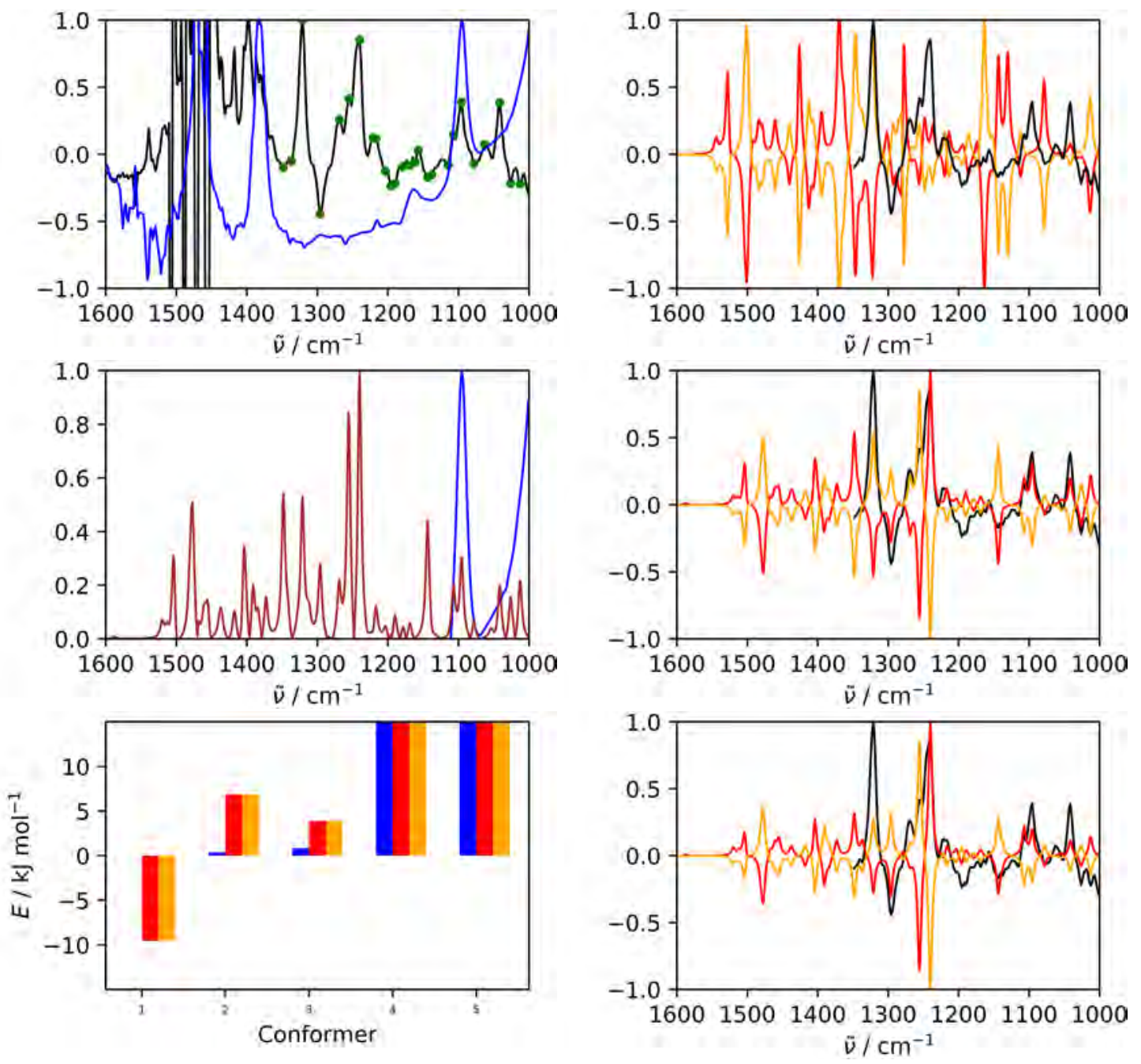

Figure S47: Compound 21, tadalafil. (Top left): Experimental VCD spectrum (black) and IR spectrum (blue). The green dots mark the peaks picked by the automatic procedure. (Top right): Unprocessed theoretical VCD spectrum of the correct enantiomer (red) and the other enantiomer (orange), as well as the experimental VCD spectrum (black). (Middle left): Experimental IR spectrum (blue) and theoretical IR (brown) obtained by taking the absolute value of the aligned VCD spectrum. (Middle right): Aligned theoretical (red, orange) and experimental VCD spectra (black) using the VSA algorithm, Boltzmann weights based on gas-phase energies. Five conformers were considered. The alignment was performed in the wavenumber range [1000, $1400 \mathrm{~cm}^{-1}$. (Bottom left): QM energy $E$ of the conformers relative to the lowest-energy conformer in the gas phase (blue), optimized for the correct enantiomer (red), and optimized for the incorrect enantiomer (orange). (Bottom right): Aligned theoretical (red, orange) and experimental VCD spectra (black) using the VSA algorithm with optimized conformer weights. 

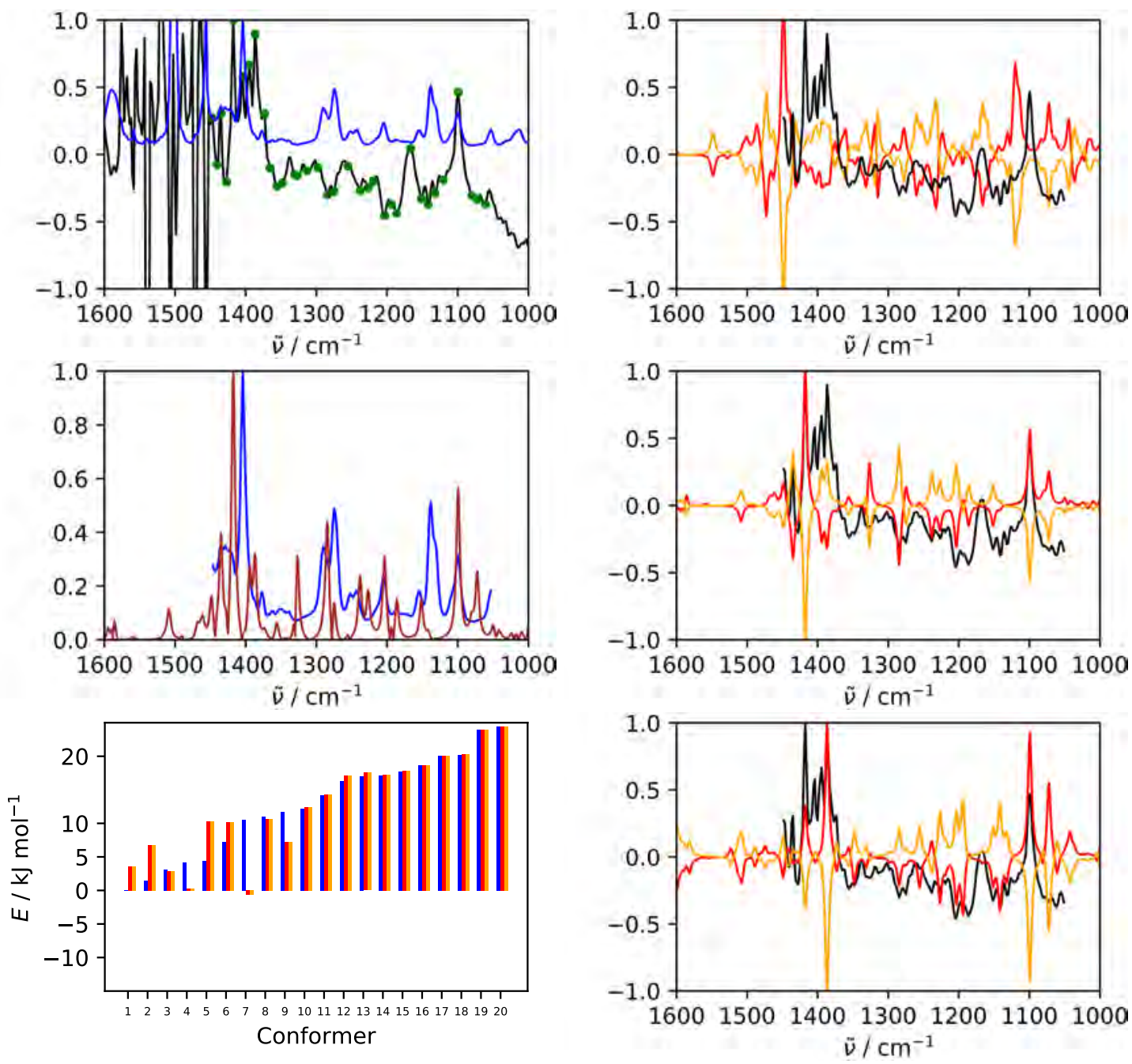

Figure S48: Compound 22, voriconazole. (Top left): Experimental VCD spectrum (black) and IR spectrum (blue). The green dots mark the peaks picked by the automatic procedure. (Top right): Unprocessed theoretical VCD spectrum of the correct enantiomer (red) and the other enantiomer (orange), as well as the experimental VCD spectrum (black). (Middle left): Experimental IR spectrum (blue) and theoretical IR (brown) obtained by taking the absolute value of the aligned VCD spectrum. (Middle right): Aligned theoretical (red, orange) and experimental VCD spectra (black) using the VSA algorithm, Boltzmann weights based on gas-phase energies. 98 conformers were considered. The alignment was performed in the wavenumber range [1050, 1450] $\mathrm{cm}^{-1}$. (Bottom left): QM energy $E$ of the first 20 conformers relative to the lowest-energy conformer in the gas phase (blue), optimized for the correct enantiomer (red), and optimized for the incorrect enantiomer (orange). (Bottom right): Aligned theoretical (red, orange) and experimental VCD spectra (black) using the VSA algorithm with optimized conformer weights. 

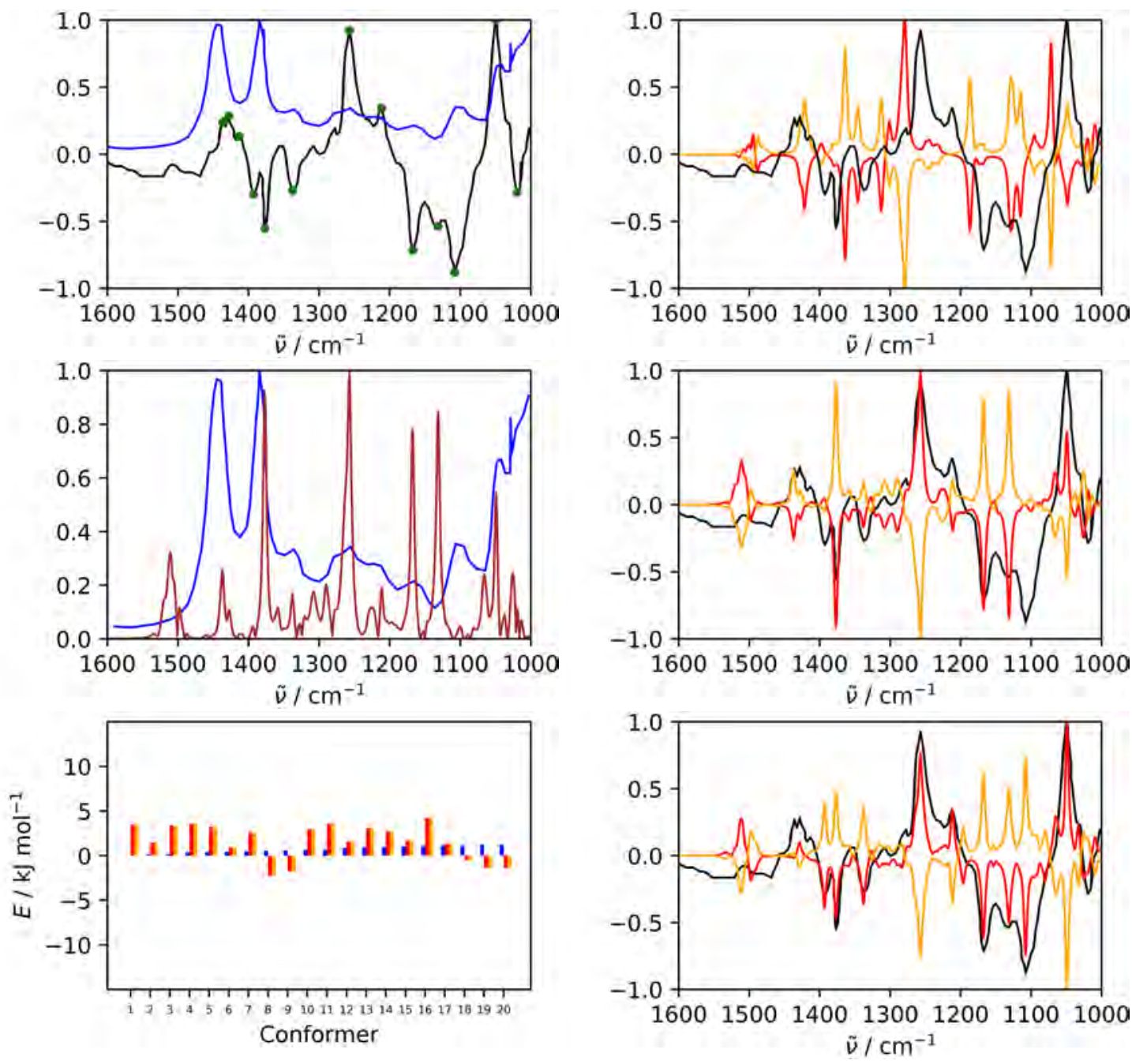

Figure S49: Compound 23, elegandiol. (Top left): Experimental VCD spectrum (black) and IR spectrum (blue) taken from Ref. [3]. The green dots mark the peaks picked by the automatic procedure. (Top right): Unprocessed theoretical VCD spectrum of the correct enantiomer (red) and the other enantiomer (orange), as well as the experimental VCD spectrum (black). (Middle left): Experimental IR spectrum (blue) and theoretical IR (brown) obtained by taking the absolute value of the aligned VCD spectrum. (Middle right): Aligned theoretical (red, orange) and experimental VCD spectra (black) using the VSA algorithm, Boltzmann weights based on gas-phase energies. 205 conformers were considered. The alignment was performed in the wavenumber range $[1000,1600] \mathrm{cm}^{-1}$. (Bottom left): QM energy $E$ of the first 20 conformers relative to the lowest-energy conformer in the gas phase (blue), optimized for the correct enantiomer (red), and optimized for the incorrect enantiomer (orange). (Bottom right): Aligned theoretical (red, orange) and experimental VCD spectra (black) using the VSA algorithm with optimized conformer weights. 

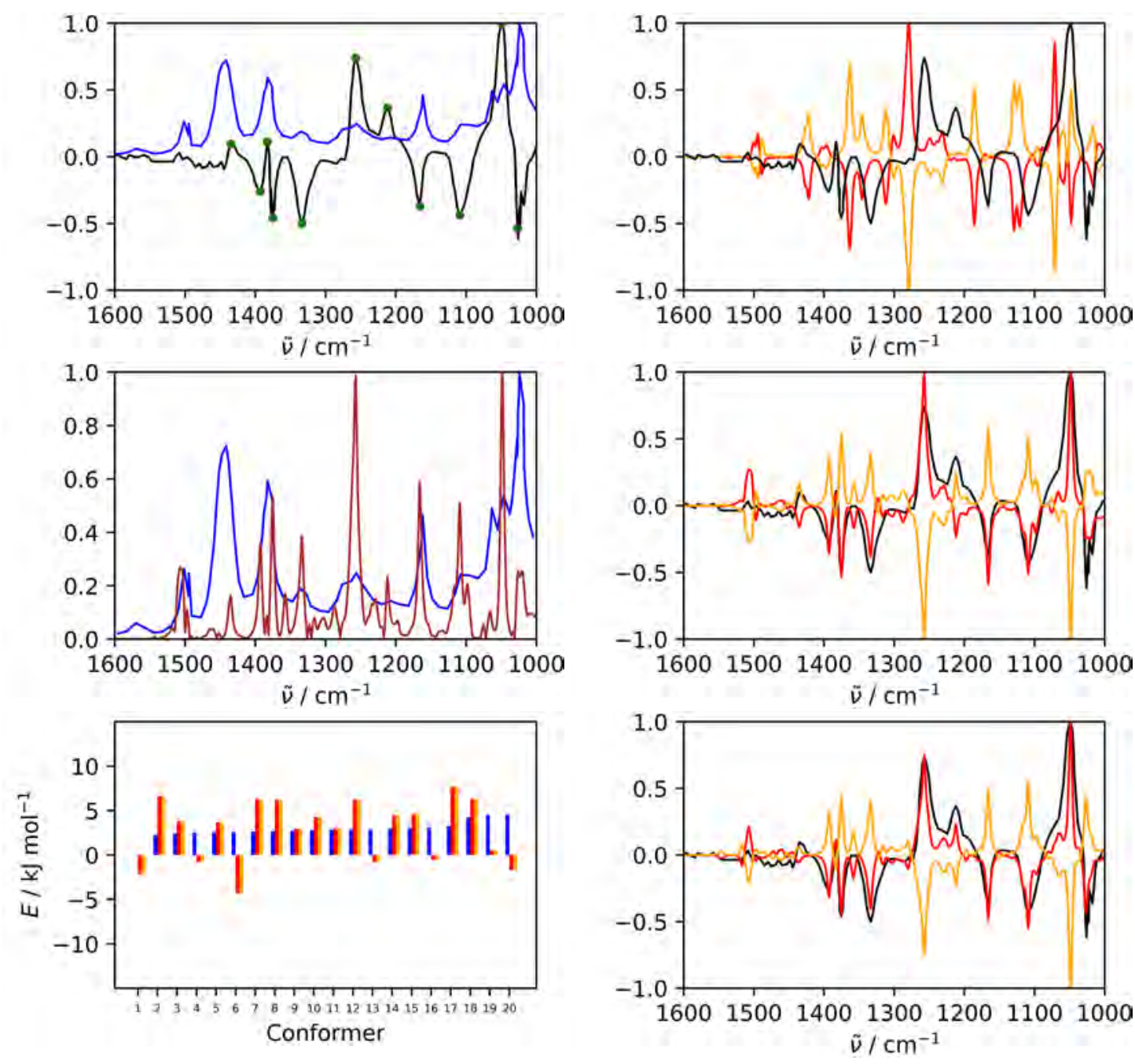

Figure S50: Compound 24, bifurcane. (Top left): Experimental VCD spectrum (black) and IR spectrum (blue) taken from Ref. [3]. The green dots mark the peaks picked by the automatic procedure. (Top right): Unprocessed theoretical VCD spectrum of the correct enantiomer (red) and the other enantiomer (orange), as well as the experimental VCD spectrum (black). (Middle left): Experimental IR spectrum (blue) and theoretical IR (brown) obtained by taking the absolute value of the aligned VCD spectrum. (Middle right): Aligned theoretical (red, orange) and experimental VCD spectra (black) using the VSA algorithm, Boltzmann weights based on gas-phase energies. 179 conformers were considered. The alignment was performed in the wavenumber range $[1000,1600] \mathrm{cm}^{-1}$. (Bottom left): QM energy $E$ of the first 20 conformers relative to the lowest-energy conformer in the gas phase (blue), optimized for the correct enantiomer (red), and optimized for the incorrect enantiomer (orange). (Bottom right): Aligned theoretical (red, orange) and experimental VCD spectra (black) using the VSA algorithm with optimized conformer weights. 

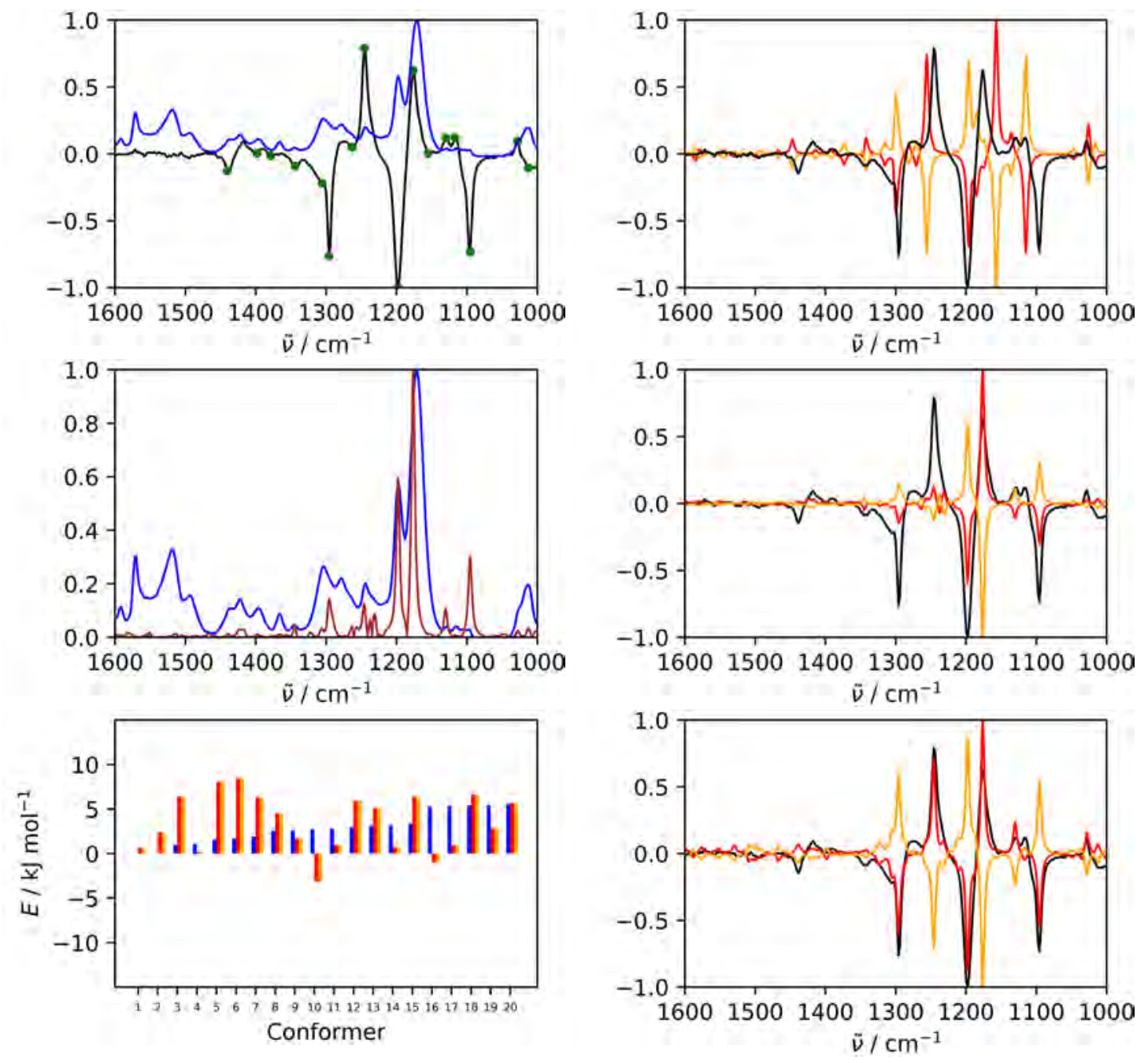

Figure S51: Compound 25, fluralaner. (Top left): Experimental VCD spectrum (black) and IR spectrum (blue) taken from Ref. [4]. The green dots mark the peaks picked by the automatic procedure. (Top right): Unprocessed theoretical VCD spectrum of the correct enantiomer (red) and the other enantiomer (orange), as well as the experimental VCD spectrum (black). (Middle left): Experimental IR spectrum (blue) and theoretical IR (brown) obtained by taking the absolute value of the aligned VCD spectrum. (Middle right): Aligned theoretical (red, orange) and experimental VCD spectra (black) using the VSA algorithm, Boltzmann weights based on gas-phase energies. 46 conformers were considered. The alignment was performed in the wavenumber range $[1000,1600] \mathrm{cm}^{-1}$. (Bottom left): QM energy $E$ of the first 20 conformers relative to the lowest-energy conformer in the gas phase (blue), optimized for the correct enantiomer (red), and optimized for the incorrect enantiomer (orange). (Bottom right): Aligned theoretical (red, orange) and experimental VCD spectra (black) using the VSA algorithm with optimized conformer weights. 


\subsection{Performance with Manual Peak Selection, Upwards Shifts Allowed}

Table S3: Performance of the VSA algorithm for conformationally flexible molecules using all conformers as measured by the Pearson coefficients $r$ for the correct (c) enantiomer, with unoptimized and optimized conformer distribution. The alignment was performed with manually picked peaks.

\begin{tabular}{ccc}
\hline Molecule & $r_{\mathrm{c}, \text { unoptimized }}$ & $r_{\mathrm{c}, \text { optimized }}$ \\
\hline \hline $\mathbf{1 5}$ & 0.57 & 0.64 \\
\hline $\mathbf{1 6}$ & 0.33 & 0.66 \\
\hline $\mathbf{1 7}$ & 0.12 & 0.74 \\
\hline $\mathbf{1 8}$ & 0.72 & 0.85 \\
\hline $\mathbf{1 9}$ & 0.59 & 0.78 \\
\hline $\mathbf{2 0}$ & 0.54 & 0.86 \\
\hline $\mathbf{2 1}$ & 0.04 & 0.10 \\
\hline $\mathbf{2 2}$ & 0.18 & 0.25 \\
\hline $\mathbf{2 3}$ & 0.59 & 0.83 \\
\hline $\mathbf{2 4}$ & 0.78 & 0.90 \\
\hline $\mathbf{2 5}$ & 0.77 & 0.93 \\
\hline
\end{tabular}



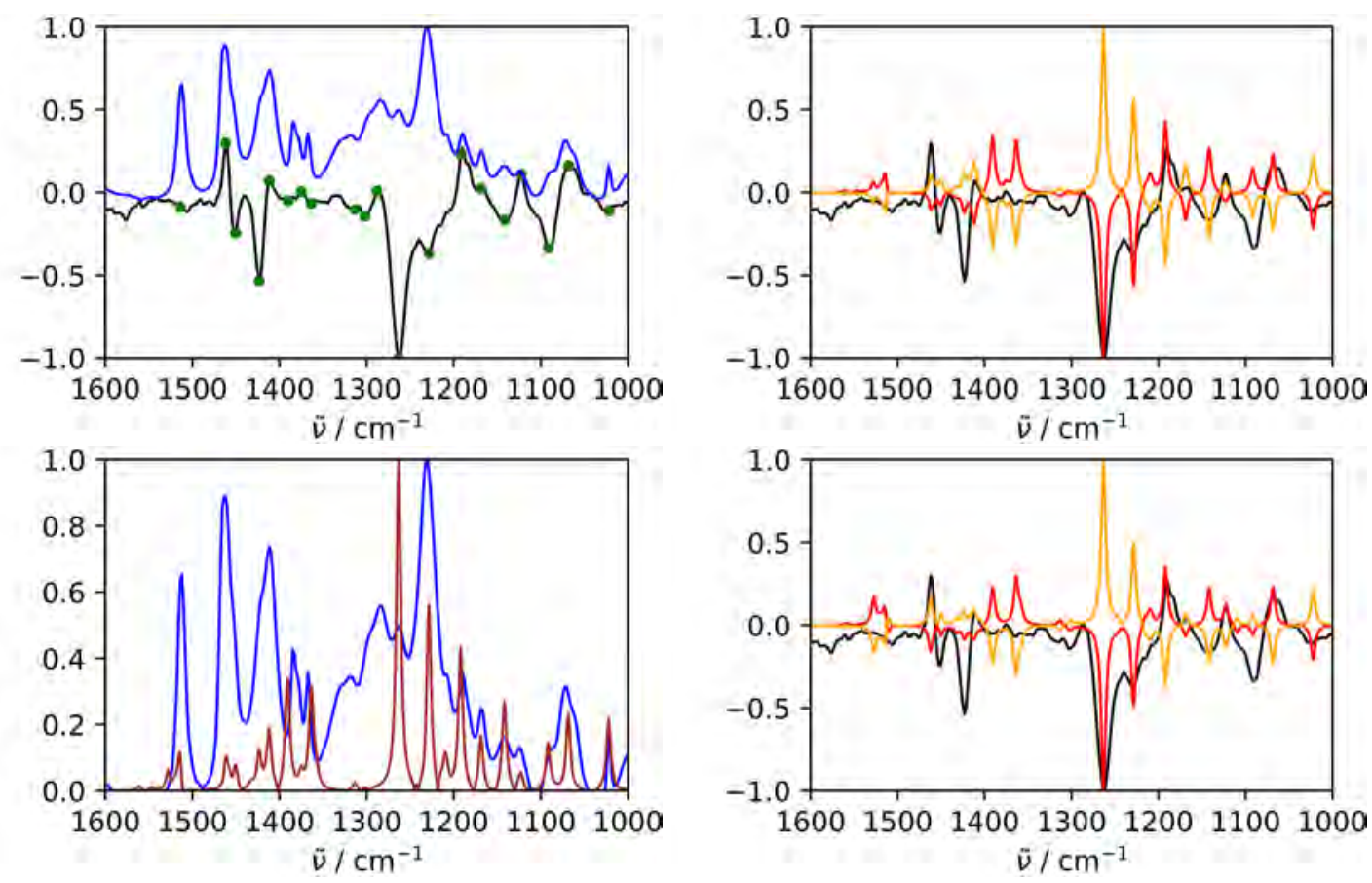

Figure S52: Compound 15, ibuprofen. (Top left): Experimental VCD spectrum (black) and IR spectrum (blue) taken from Ref. [1]. The green dots mark the peaks picked manually. (Top right): Aligned theoretical VCD spectrum of the correct enantiomer (red) and the other enantiomer (orange) and experimental VCD spectra (black) using the VSA algorithm, Boltzmann weights based on gas-phase energies. 12 conformers were considered. The alignment was performed in the wavenumber range $[1000,1600] \mathrm{cm}^{-1}$. (Bottom left): Experimental IR spectrum (blue) and theoretical IR (brown) obtained by taking the absolute value of the aligned VCD spectrum. (Bottom right): Aligned theoretical (red, orange) and experimental VCD spectra (black) using the VSA algorithm with optimized conformer weights. 

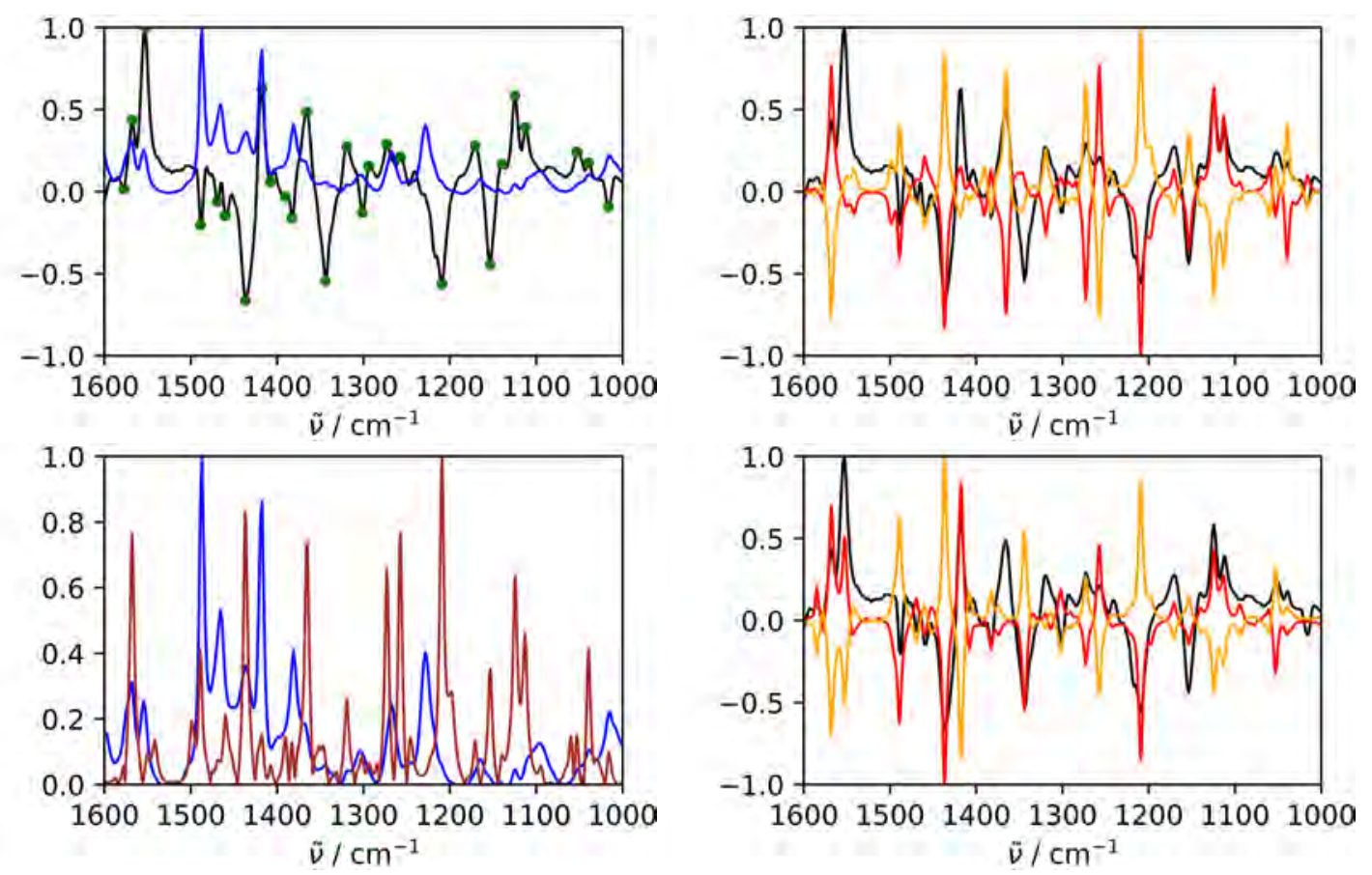

Figure S53: Compound 16, filorexant. (Top left): Experimental VCD spectrum (black) and IR spectrum (blue) taken from Ref. [1]. The green dots mark the peaks picked manually. (Top right): Aligned theoretical VCD spectrum of the correct enantiomer (red) and the other enantiomer (orange) and experimental VCD spectra (black) using the VSA algorithm, Boltzmann weights based on gas-phase energies. 126 conformers were considered. The alignment was performed in the wavenumber range $[1000,1600] \mathrm{cm}^{-1}$. (Bottom left): Experimental IR spectrum (blue) and theoretical IR (brown) obtained by taking the absolute value of the aligned VCD spectrum. (Bottom right): Aligned theoretical (red, orange) and experimental VCD spectra (black) using the VSA algorithm with optimized conformer weights. 

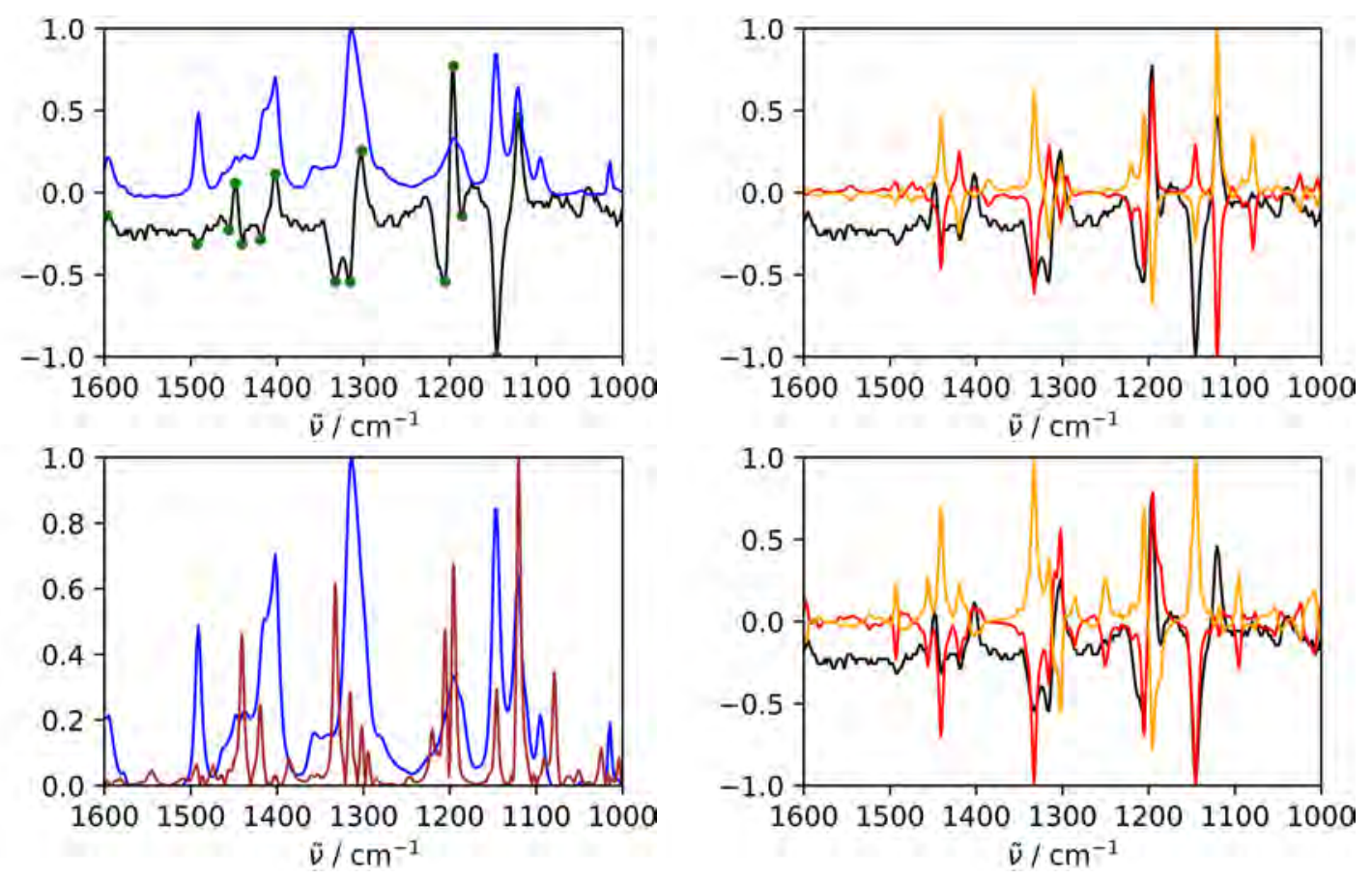

Figure S54: Compound 17, laropiprant. (Top left): Experimental VCD spectrum (black) and IR spectrum (blue) taken from Ref. [1]. The green dots mark the peaks picked manually. (Top right): Aligned theoretical VCD spectrum of the correct enantiomer (red) and the other enantiomer (orange) and experimental VCD spectra (black) using the VSA algorithm, Boltzmann weights based on gas-phase energies. 73 conformers were considered. The alignment was performed in the wavenumber range $[1000,1600] \mathrm{cm}^{-1}$. (Bottom left): Experimental IR spectrum (blue) and theoretical IR (brown) obtained by taking the absolute value of the aligned VCD spectrum. (Bottom right): Aligned theoretical (red, orange) and experimental VCD spectra (black) using the VSA algorithm with optimized conformer weights. 

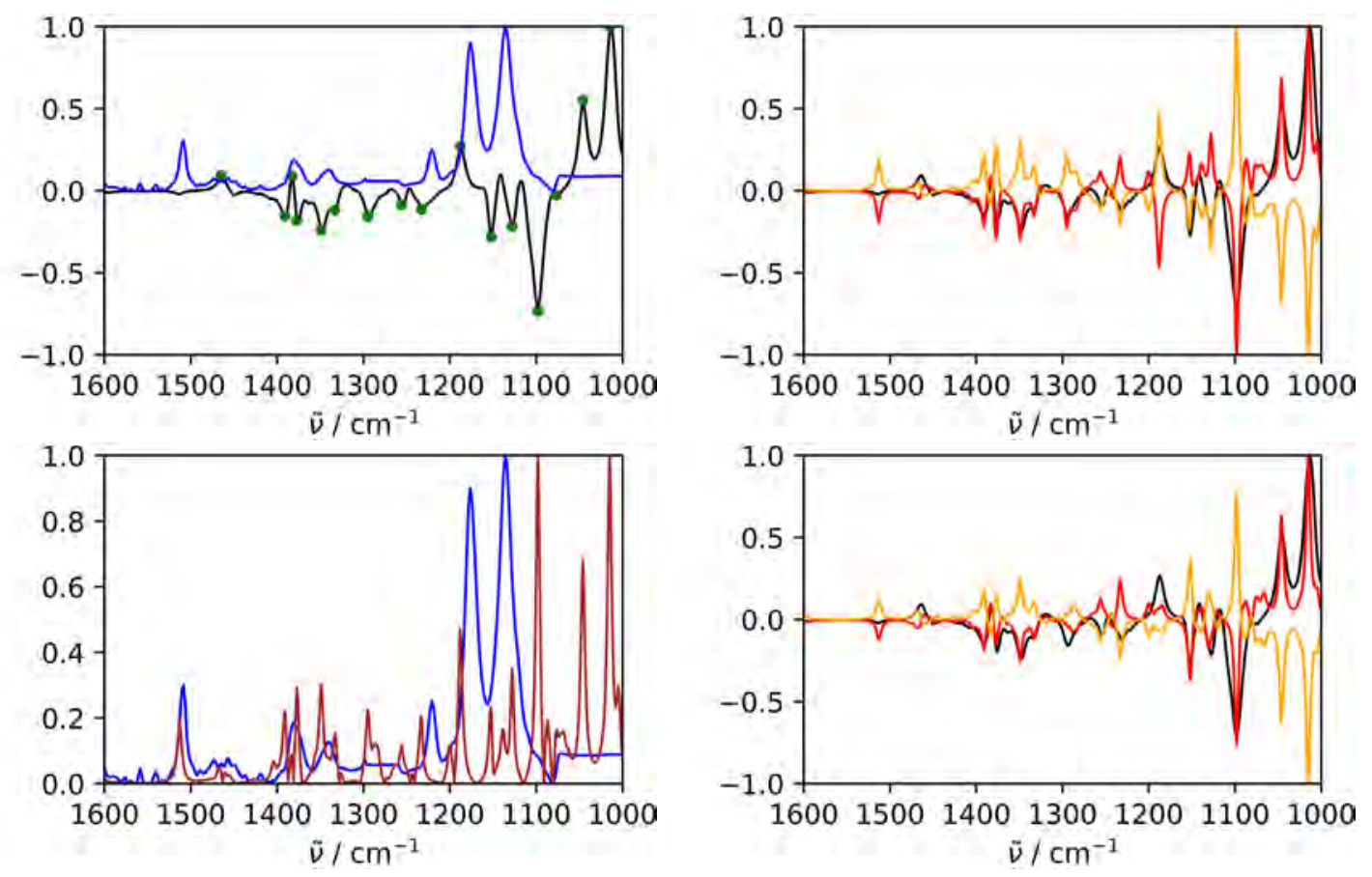

Figure S55: Compound 18, aprepitant. (Top left): Experimental VCD spectrum (black) and IR spectrum (blue) taken from Ref. [1]. The green dots mark the peaks picked manually. (Top right): Aligned theoretical VCD spectrum of the correct enantiomer (red) and the other enantiomer (orange) and experimental VCD spectra (black) using the VSA algorithm, Boltzmann weights based on gas-phase energies. 141 conformers were considered. The alignment was performed in the wavenumber range $[1000,1600] \mathrm{cm}^{-1}$. (Bottom left): Experimental IR spectrum (blue) and theoretical IR (brown) obtained by taking the absolute value of the aligned VCD spectrum. (Bottom right): Aligned theoretical (red, orange) and experimental VCD spectra (black) using the VSA algorithm with optimized conformer weights. 



Figure S56: Compound 19, ezetimibe. (Top left): Experimental VCD spectrum (black) and IR spectrum (blue) taken from Ref. [1]. The green dots mark the peaks picked manually. (Top right): Aligned theoretical VCD spectrum of the correct enantiomer (red) and the other enantiomer (orange) and experimental VCD spectra (black) using the VSA algorithm, Boltzmann weights based on gas-phase energies. 74 conformers were considered. The alignment was performed in the wavenumber range $[1150,1450] \mathrm{cm}^{-1}$. (Bottom left): Experimental IR spectrum (blue) and theoretical IR (brown) obtained by taking the absolute value of the aligned VCD spectrum. (Bottom right): Aligned theoretical (red, orange) and experimental VCD spectra (black) using the VSA algorithm with optimized conformer weights. 

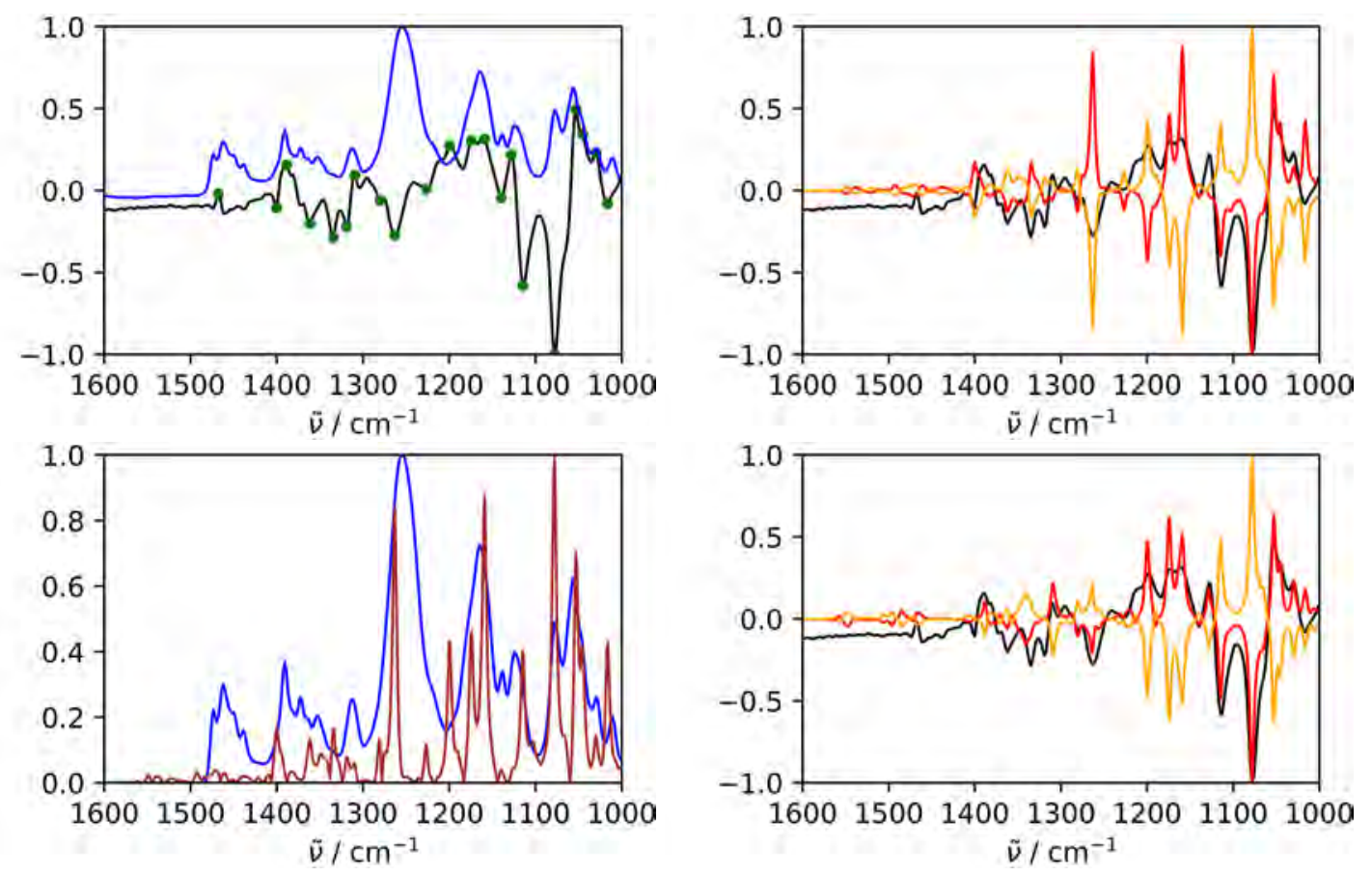

Figure S57: Compound 20, simvastatin. (Top left): Experimental VCD spectrum (black) and IR spectrum (blue) taken from Ref. [1]. The green dots mark the peaks picked manually. (Top right): Aligned theoretical VCD spectrum of the correct enantiomer (red) and the other enantiomer (orange) and experimental VCD spectra (black) using the VSA algorithm, Boltzmann weights based on gas-phase energies. 202 conformers were considered. The alignment was performed in the wavenumber range $[1000,1600] \mathrm{cm}^{-1}$. (Bottom left): Experimental IR spectrum (blue) and theoretical IR (brown) obtained by taking the absolute value of the aligned VCD spectrum. (Bottom right): Aligned theoretical (red, orange) and experimental VCD spectra (black) using the VSA algorithm with optimized conformer weights. 

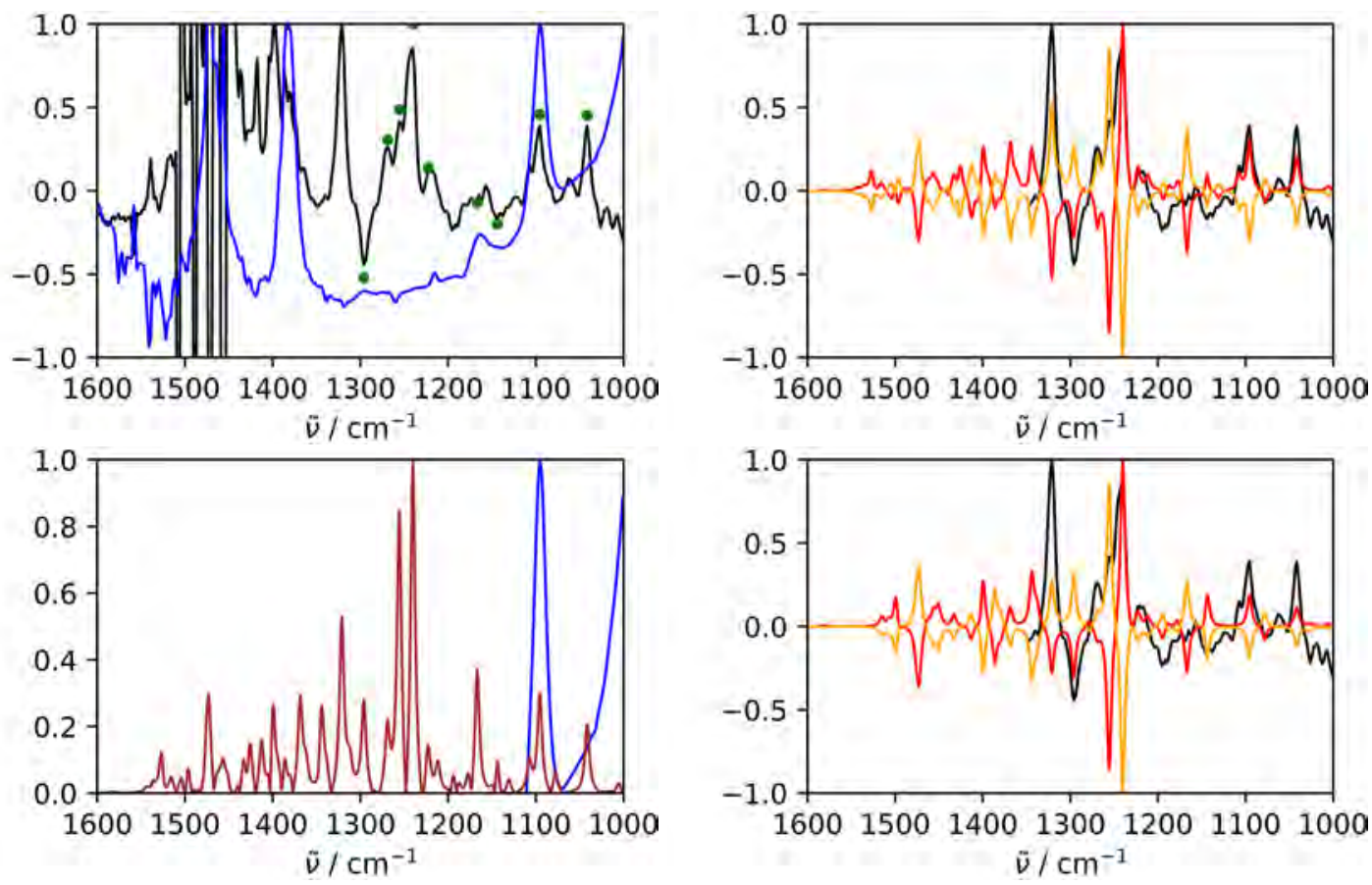

Figure S58: Compound 21, tadalafil. (Top left): Experimental VCD spectrum (black) and IR spectrum (blue). The green dots mark the peaks picked manually. (Top right): Aligned theoretical VCD spectrum of the correct enantiomer (red) and the other enantiomer (orange) and experimental VCD spectra (black) using the VSA algorithm, Boltzmann weights based on gas-phase energies. Five conformers were considered. The alignment was performed in the wavenumber range [1000, 1350] $\mathrm{cm}^{-1}$. (Bottom left): Experimental IR spectrum (blue) and theoretical IR (brown) obtained by taking the absolute value of the aligned VCD spectrum. (Bottom right): Aligned theoretical (red, orange) and experimental VCD spectra (black) using the VSA algorithm with optimized conformer weights. 



Figure S59: Compound 22, voriconazole. (Top left): Experimental VCD spectrum (black) and IR spectrum (blue). The green dots mark the peaks picked manually. (Top right): Aligned theoretical VCD spectrum of the correct enantiomer (red) and the other enantiomer (orange) and experimental VCD spectra (black) using the VSA algorithm, Boltzmann weights based on gas-phase energies. 98 conformers were considered. The alignment was performed in the wavenumber range $[1050,1450] \mathrm{cm}^{-1}$. (Bottom left): Experimental IR spectrum (blue) and theoretical IR (brown) obtained by taking the absolute value of the aligned VCD spectrum. (Bottom right): Aligned theoretical (red, orange) and experimental VCD spectra (black) using the VSA algorithm with optimized conformer weights. 

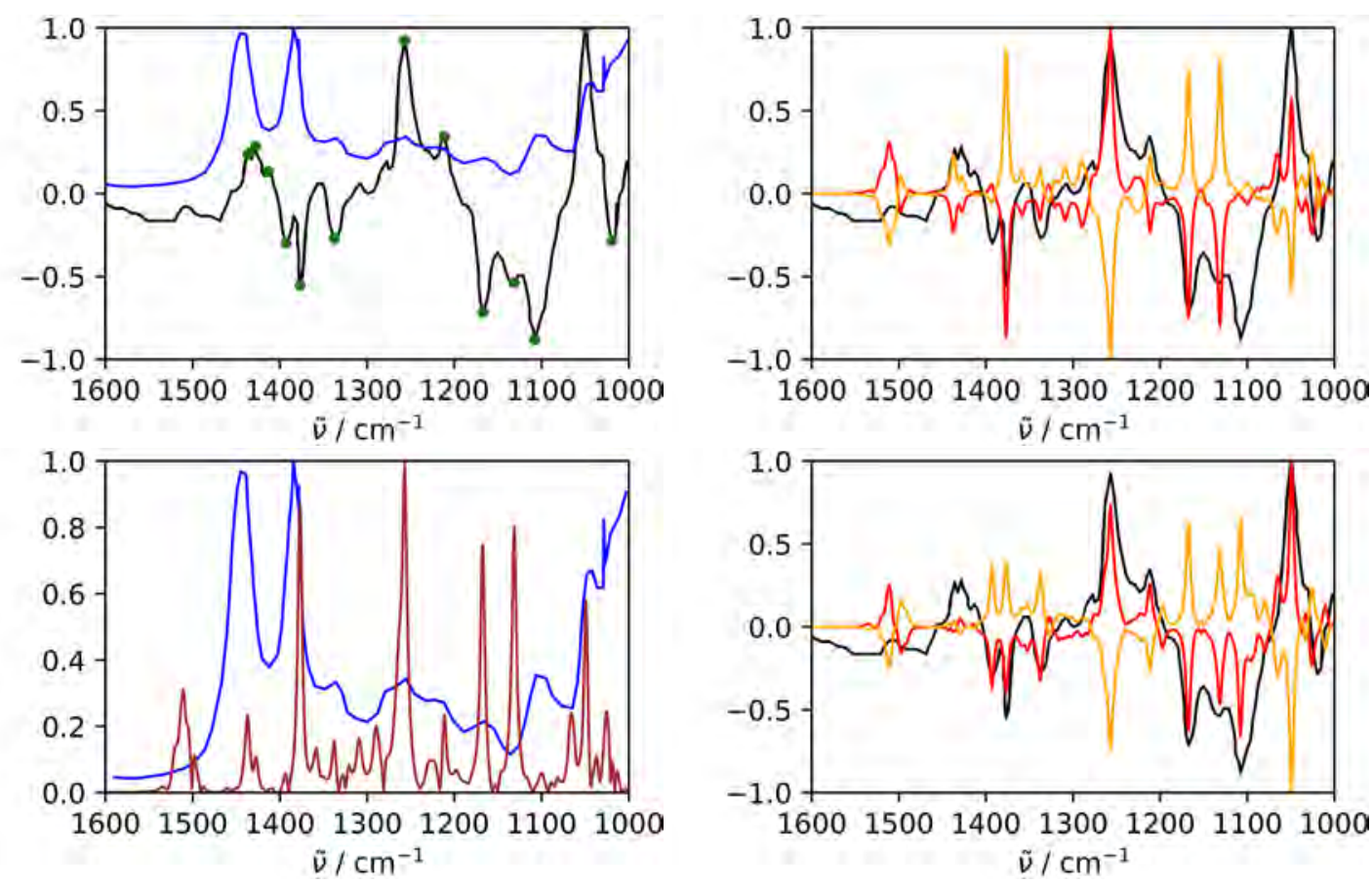

Figure S60: Compound 23, elegandiol. (Top left): Experimental VCD spectrum (black) and IR spectrum (blue). The green dots mark the peaks picked manually. (Top right): Aligned theoretical VCD spectrum of the correct enantiomer (red) and the other enantiomer (orange) and experimental VCD spectra (black) using the VSA algorithm, Boltzmann weights based on gas-phase energies. 205 conformers were considered. The alignment was performed in the wavenumber range $[1000,1600] \mathrm{cm}^{-1}$. (Bottom left): Experimental IR spectrum (blue) and theoretical IR (brown) obtained by taking the absolute value of the aligned VCD spectrum. (Bottom right): Aligned theoretical (red, orange) and experimental VCD spectra (black) using the VSA algorithm with optimized conformer weights. 

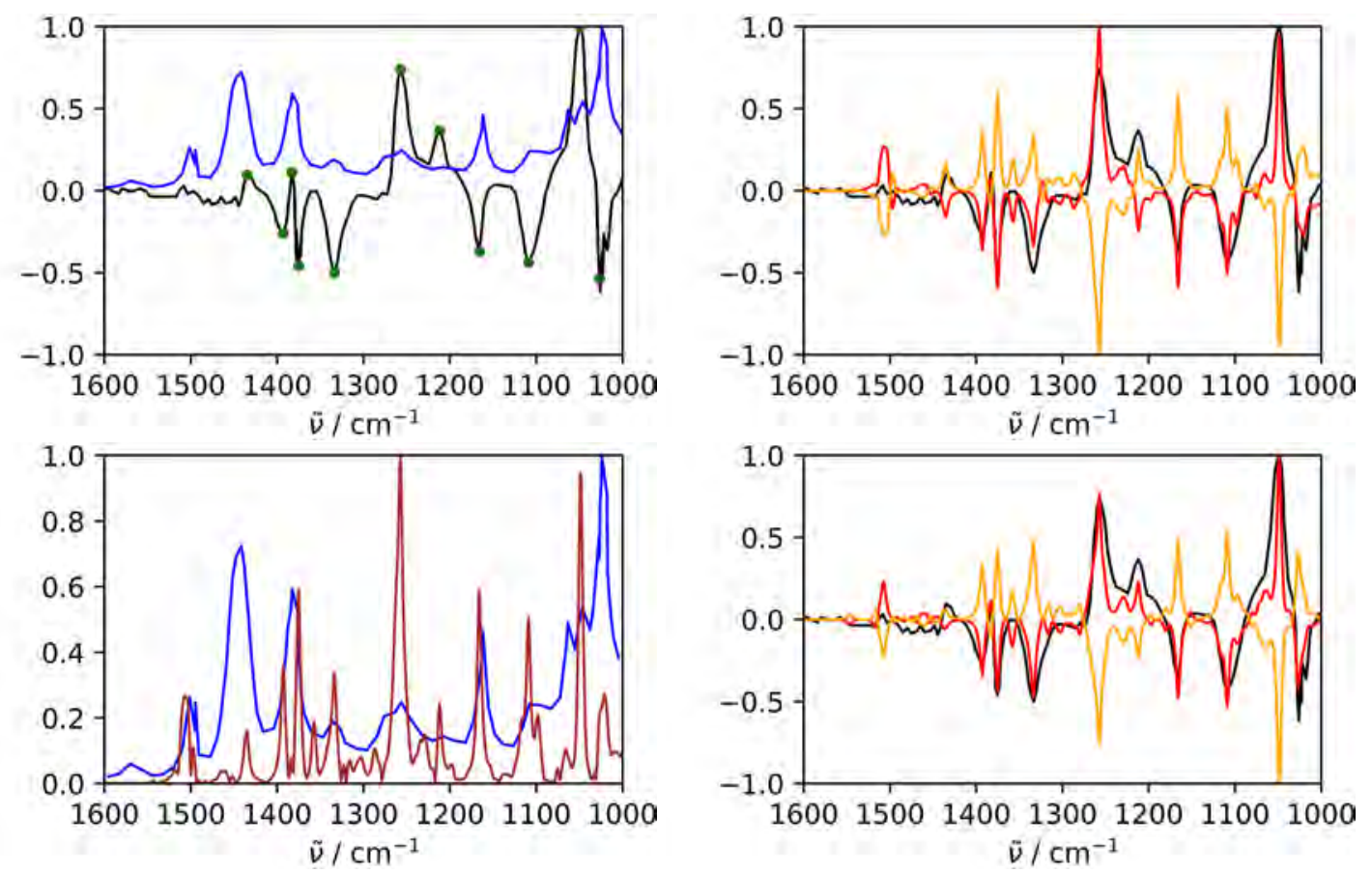

Figure S61: Compound 24, bifurcane. (Top left): Experimental VCD spectrum (black) and IR spectrum (blue). The green dots mark the peaks picked manually. (Top right): Aligned theoretical VCD spectrum of the correct enantiomer (red) and the other enantiomer (orange) and experimental VCD spectra (black) using the VSA algorithm, Boltzmann weights based on gas-phase energies. 179 conformers were considered. The alignment was performed in the wavenumber range $[1000,1600] \mathrm{cm}^{-1}$. (Bottom left): Experimental IR spectrum (blue) and theoretical IR (brown) obtained by taking the absolute value of the aligned VCD spectrum. (Bottom right): Aligned theoretical (red, orange) and experimental VCD spectra (black) using the VSA algorithm with optimized conformer weights. 



Figure S62: Compound 25, fluralaner. (Top left): Experimental VCD spectrum (black) and IR spectrum (blue). The green dots mark the peaks picked manually. (Top right): Aligned theoretical VCD spectrum of the correct enantiomer (red) and the other enantiomer (orange) and experimental VCD spectra (black) using the VSA algorithm, Boltzmann weights based on gas-phase energies. 46 conformers were considered. The alignment was performed in the wavenumber range $[1000,1600] \mathrm{cm}^{-1}$. (Bottom left): Experimental IR spectrum (blue) and theoretical IR (brown) obtained by taking the absolute value of the aligned VCD spectrum. (Bottom right): Aligned theoretical (red, orange) and experimental VCD spectra (black) using the VSA algorithm with optimized conformer weights.

\subsection{Convergence}


Figure S63: Unoptimized (left) and optimized (right) Pearson coefficient $r$ for correct enantiomer (red) and wrong enantiomer (orange) for compound 16, filorexant, as a function of the number of conformers included. 

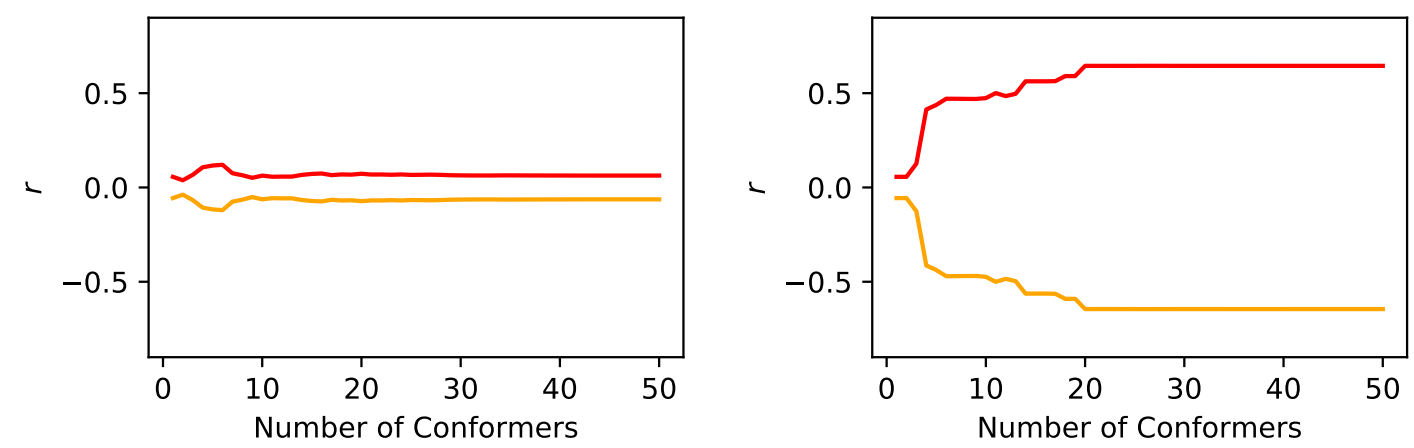

Figure S64: Unoptimized (left) and optimized (right) Pearson coefficient $r$ for correct enantiomer (red) and wrong enantiomer (orange) for compound 17, laropiprant, as a function of the number of conformers included.
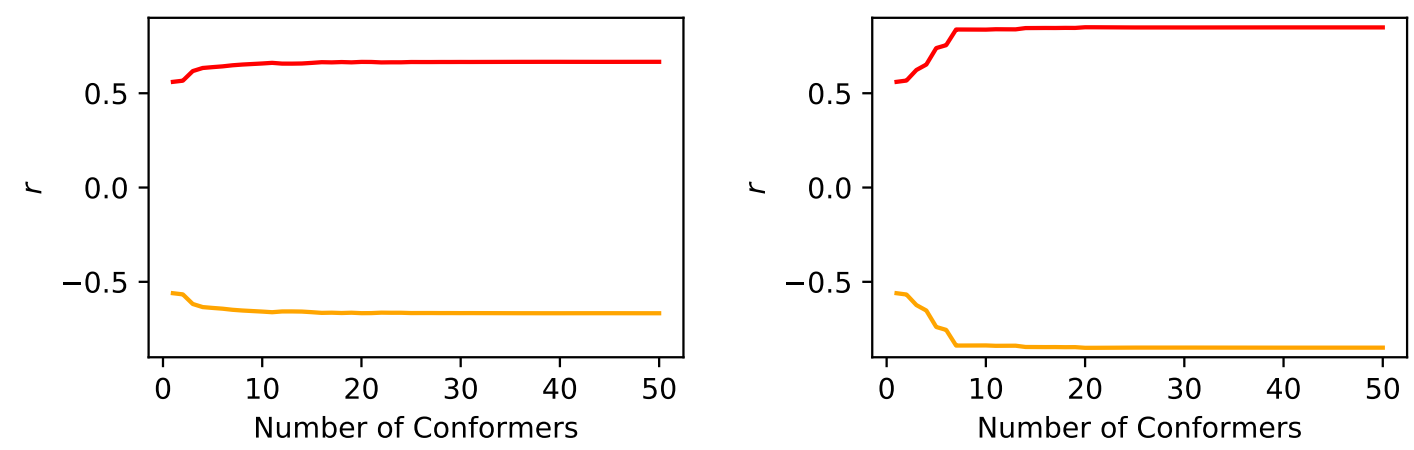

Figure S65: Unoptimized (left) and optimized (right) Pearson coefficient $r$ for correct enantiomer (red) and wrong enantiomer (orange) for compound 18, aprepitant, as a function of the number of conformers included.


Figure S66: Unoptimized (left) and optimized (right) Pearson coefficient $r$ for correct enantiomer (red) and wrong enantiomer (orange) for compound 19, ezetimibe, as a function of the number of conformers included. 

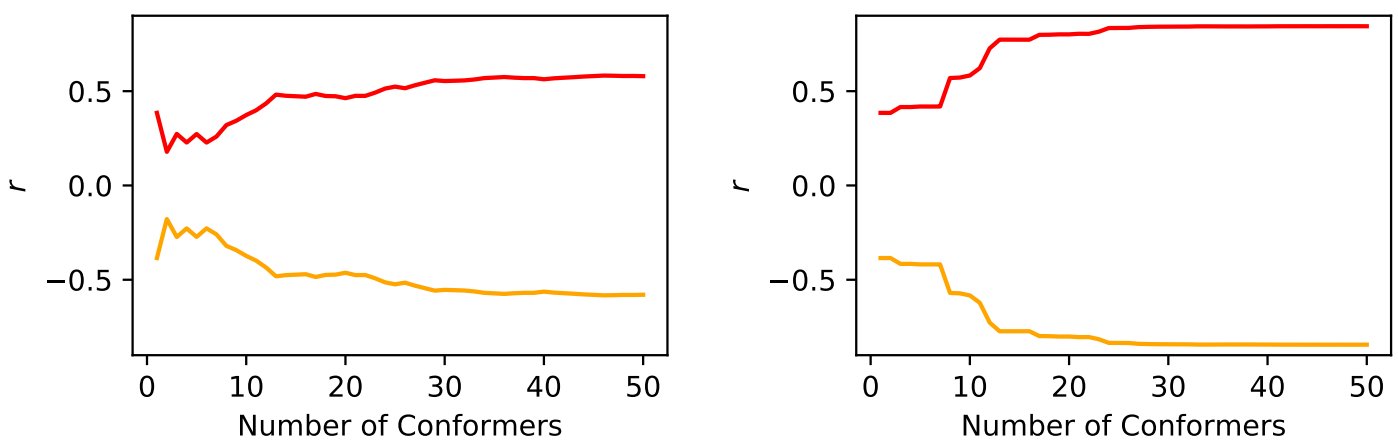

Figure S67: Unoptimized (left) and optimized (right) Pearson coefficient $r$ for correct enantiomer (red) and wrong enantiomer (orange) for compound $\mathbf{2 0}$, simvastatin, as a function of the number of conformers included.
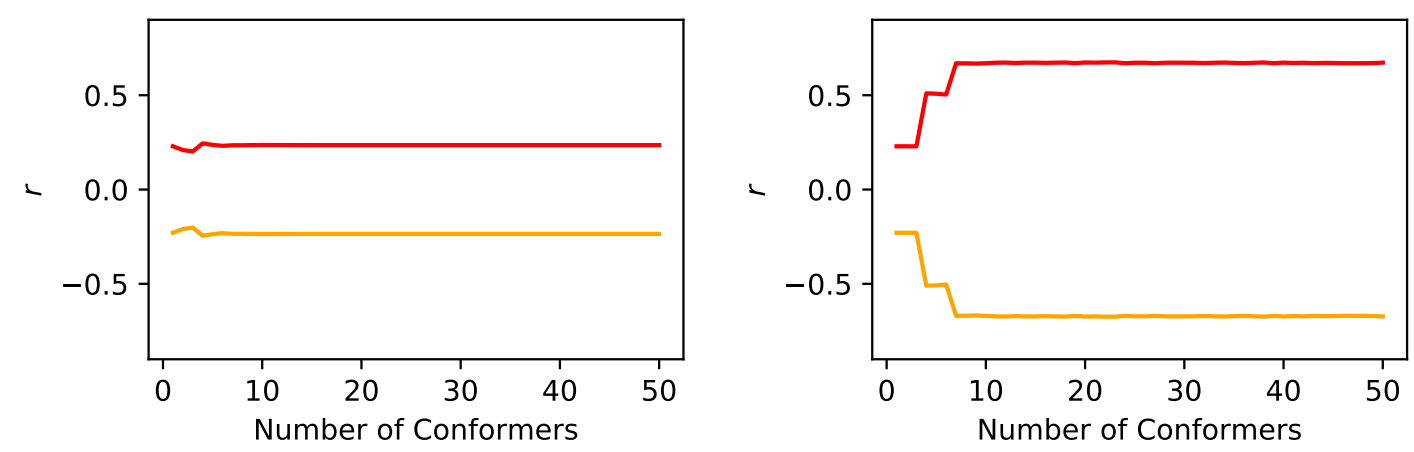

Figure S68: Unoptimized (left) and optimized (right) Pearson coefficient $r$ for correct enantiomer (red) and wrong enantiomer (orange) for compound 22, voriconazole, as a function of the number of conformers included.
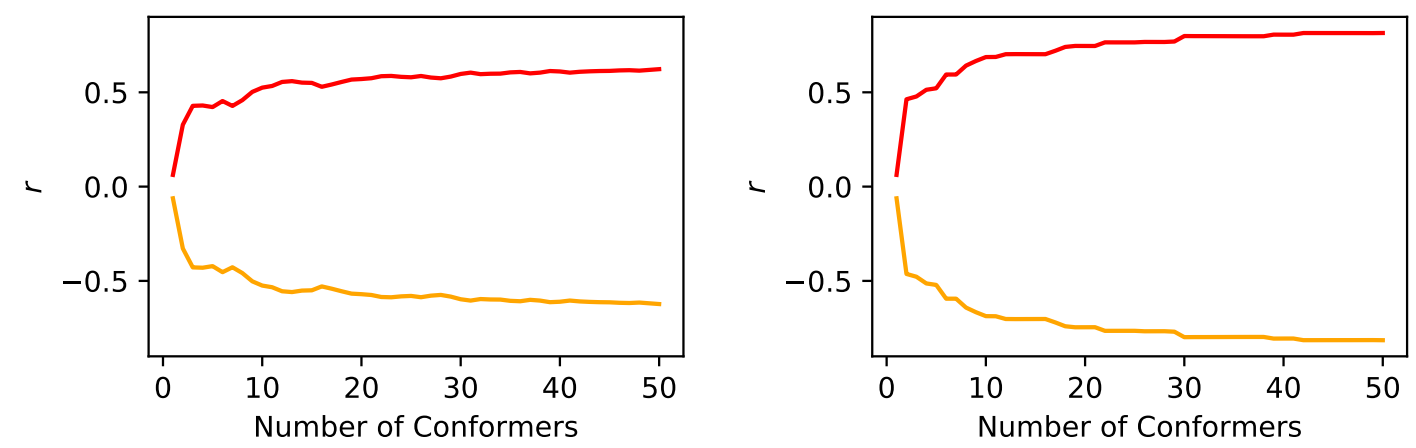

Figure S69: Unoptimized (left) and optimized (right) Pearson coefficient $r$ for correct enantiomer (red) and wrong enantiomer (orange) for compound $\mathbf{2 3}$, elegandiol, as a function of the number of conformers included. 

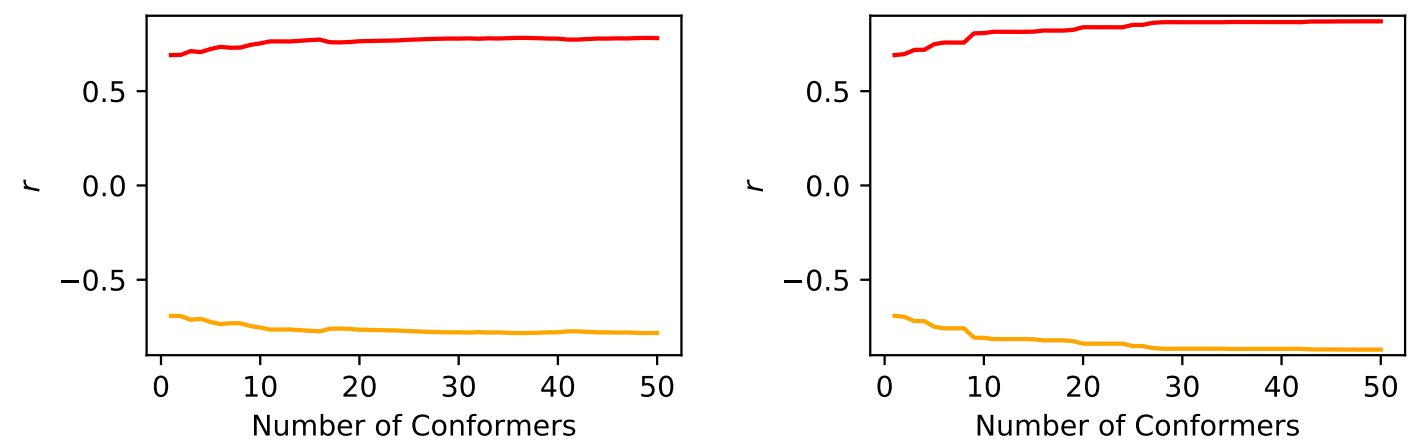

Figure S70: Unoptimized (left) and optimized (right) Pearson coefficient $r$ for correct enantiomer (red) and wrong enantiomer (orange) for compound 24, bifurcane, as a function of the number of conformers included.
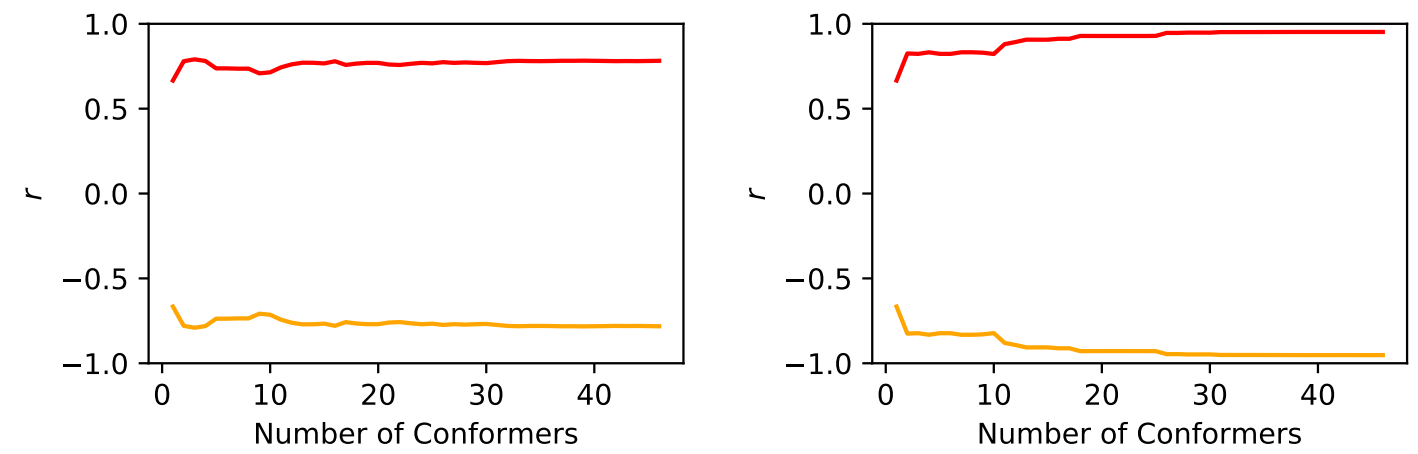

Figure S71: Unoptimized (left) and optimized (right) Pearson coefficient $r$ for correct enantiomer (red) and wrong enantiomer (orange) for compound 25, fluralaner, as a function of the number of conformers included. 


\section{Conformationally Flexible Molecules - 20 Conformers}

\subsection{Performance with Automatic Peak Selection, Upwards Shifts Allowed}


Figure S72: Compound 16, filorexant. (Left): Aligned theoretical VCD spectrum of the correct enantiomer (red) and the other enantiomer (orange) and experimental VCD spectra (black) using the VSA algorithm, Boltzmann weights based on gas-phase energies. 20 conformers were considered. The alignment was performed in the wavenumber range $[1000,1600] \mathrm{cm}^{-1}$. (Right): Aligned theoretical (red, orange) and experimental VCD spectra (black) using the VSA algorithm with optimized conformer weights.
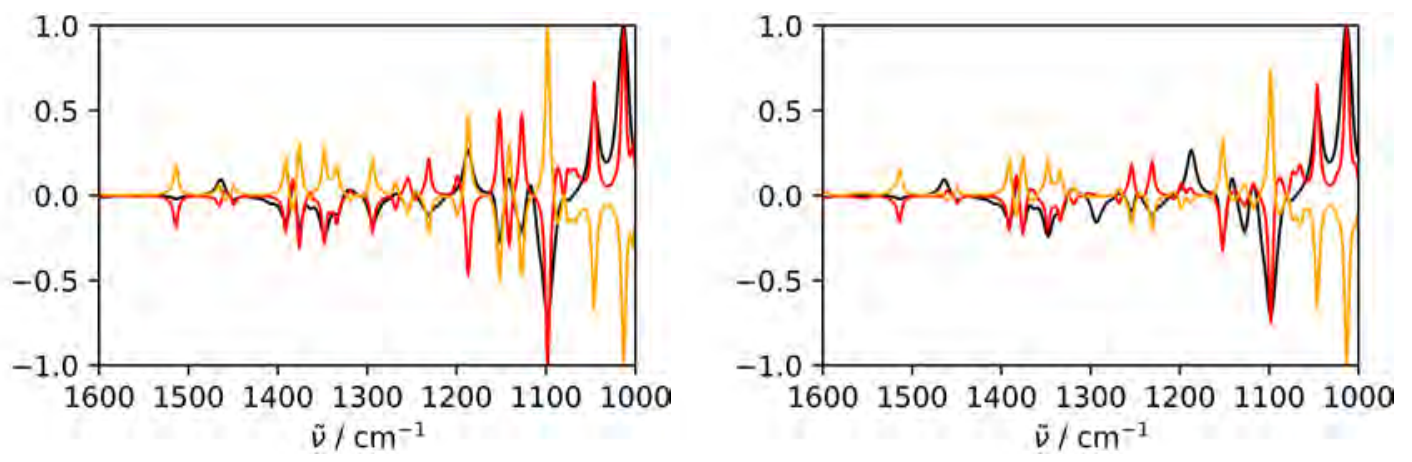

Figure S73: Compound 18, aprepitant. (Left): Aligned theoretical VCD spectrum of the correct enantiomer (red) and the other enantiomer (orange) and experimental VCD spectra (black) using the VSA algorithm, Boltzmann weights based on gas-phase energies. 20 conformers were considered. The alignment was performed in the wavenumber range $[1000,1600] \mathrm{cm}^{-1}$. (Right): Aligned theoretical (red, orange) and experimental VCD spectra (black) using the VSA algorithm with optimized conformer weights. 

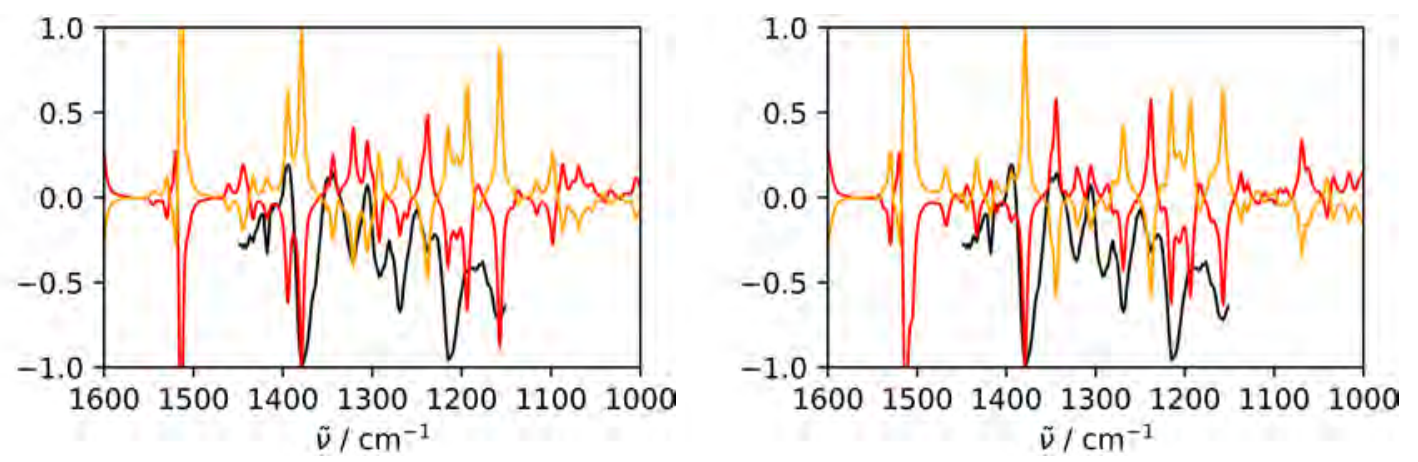

Figure S74: Compound 19, ezetimibe. (Left): Aligned theoretical VCD spectrum of the correct enantiomer (red) and the other enantiomer (orange) and experimental VCD spectra (black) using the VSA algorithm, Boltzmann weights based on gas-phase energies. 20 conformers were considered. The alignment was performed in the wavenumber range $[1150,1450] \mathrm{cm}^{-1}$. (Right): Aligned theoretical (red, orange) and experimental VCD spectra (black) using the VSA algorithm with optimized conformer weights.
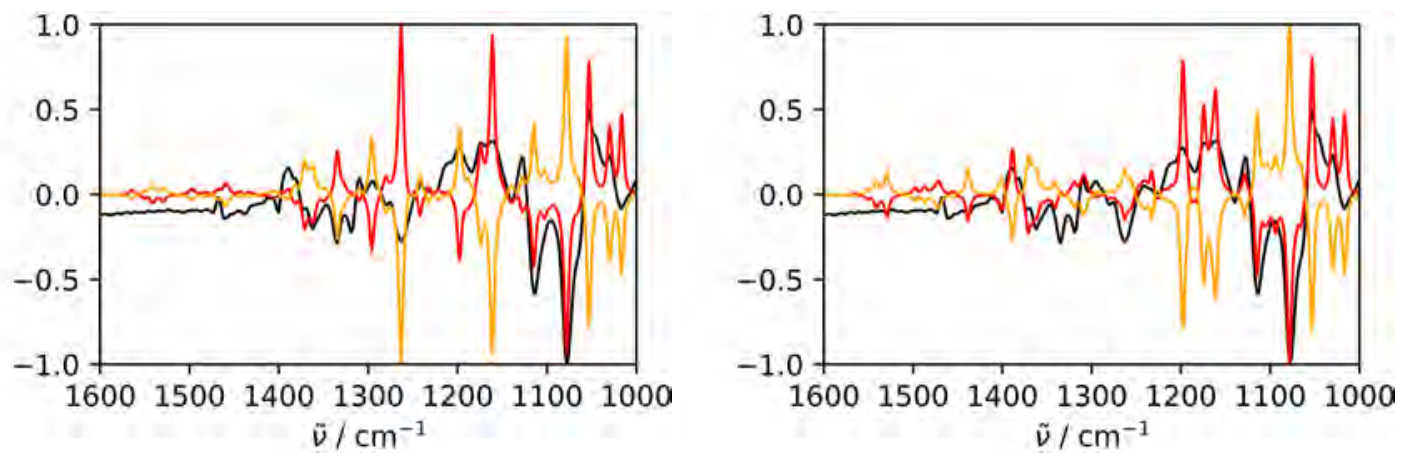

Figure S75: Compound 20, simvastatin. (Left): Aligned theoretical VCD spectrum of the correct enantiomer (red) and the other enantiomer (orange) and experimental VCD spectra (black) using the VSA algorithm, Boltzmann weights based on gas-phase energies. 20 conformers were considered. The alignment was performed in the wavenumber range $[1000,1600] \mathrm{cm}^{-1}$. (Right): Aligned theoretical (red, orange) and experimental VCD spectra (black) using the VSA algorithm with optimized conformer weights. 

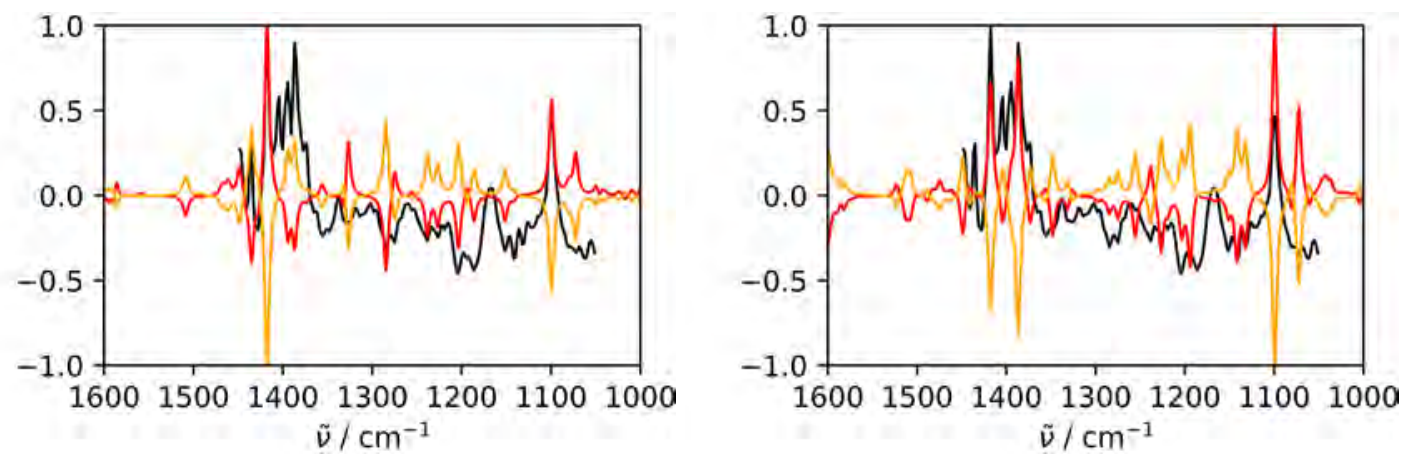

Figure S76: Compound 22, voriconazole. (Left): Aligned theoretical VCD spectrum of the correct enantiomer (red) and the other enantiomer (orange) and experimental VCD spectra (black) using the VSA algorithm, Boltzmann weights based on gas-phase energies. 20 conformers were considered. The alignment was performed in the wavenumber range $[1050,1450] \mathrm{cm}^{-1}$. (Right): Aligned theoretical (red, orange) and experimental VCD spectra (black) using the VSA algorithm with optimized conformer weights.
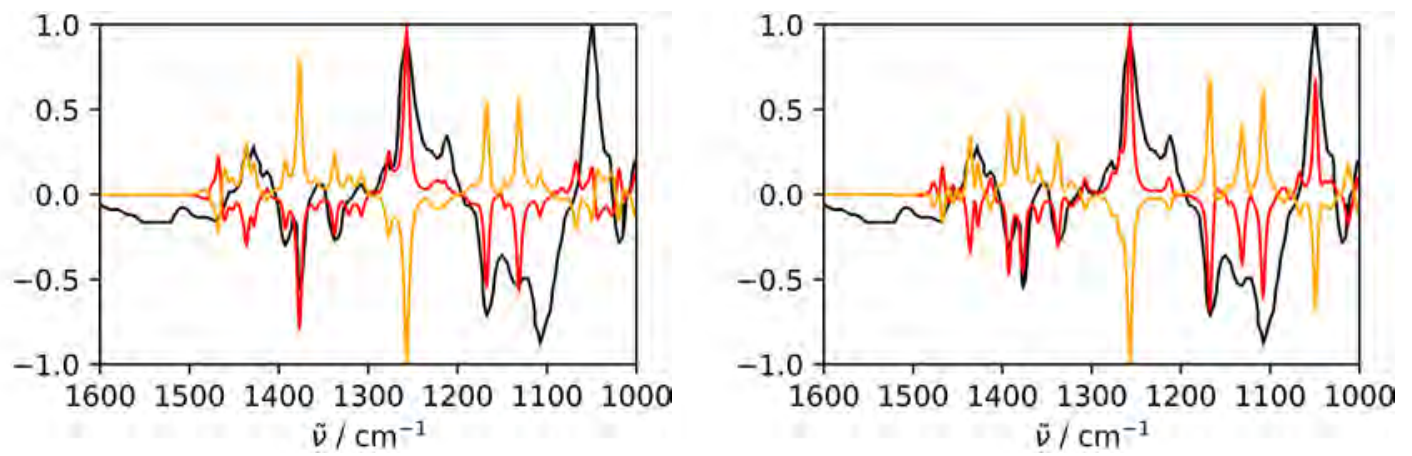

Figure S77: Compound 23, elegandiol. (Left): Aligned theoretical VCD spectrum of the correct enantiomer (red) and the other enantiomer (orange) and experimental VCD spectra (black) using the VSA algorithm, Boltzmann weights based on gas-phase energies. 20 conformers were considered. The alignment was performed in the wavenumber range $[1000,1600] \mathrm{cm}^{-1}$. (Right): Aligned theoretical (red, orange) and experimental VCD spectra (black) using the VSA algorithm with optimized conformer weights. 

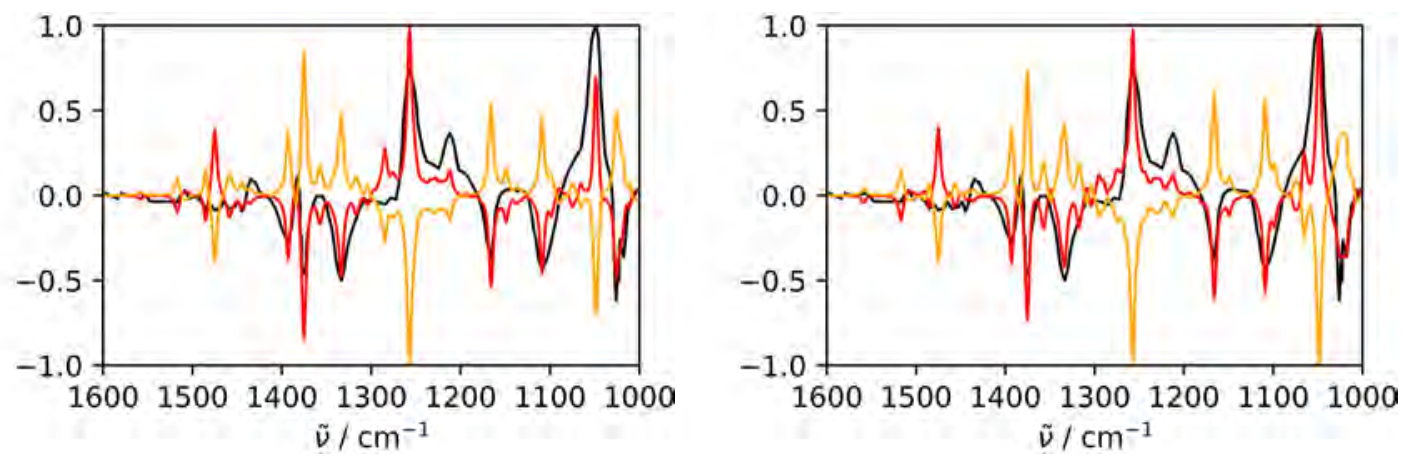

Figure S78: Compound 24, bifurcane. (Left): Aligned theoretical VCD spectrum of the correct enantiomer (red) and the other enantiomer (orange) and experimental VCD spectra (black) using the VSA algorithm, Boltzmann weights based on gas-phase energies. 20 conformers were considered. The alignment was performed in the wavenumber range $[1000,1600] \mathrm{cm}^{-1}$. (Right): Aligned theoretical (red, orange) and experimental VCD spectra (black) using the VSA algorithm with optimized conformer weights.
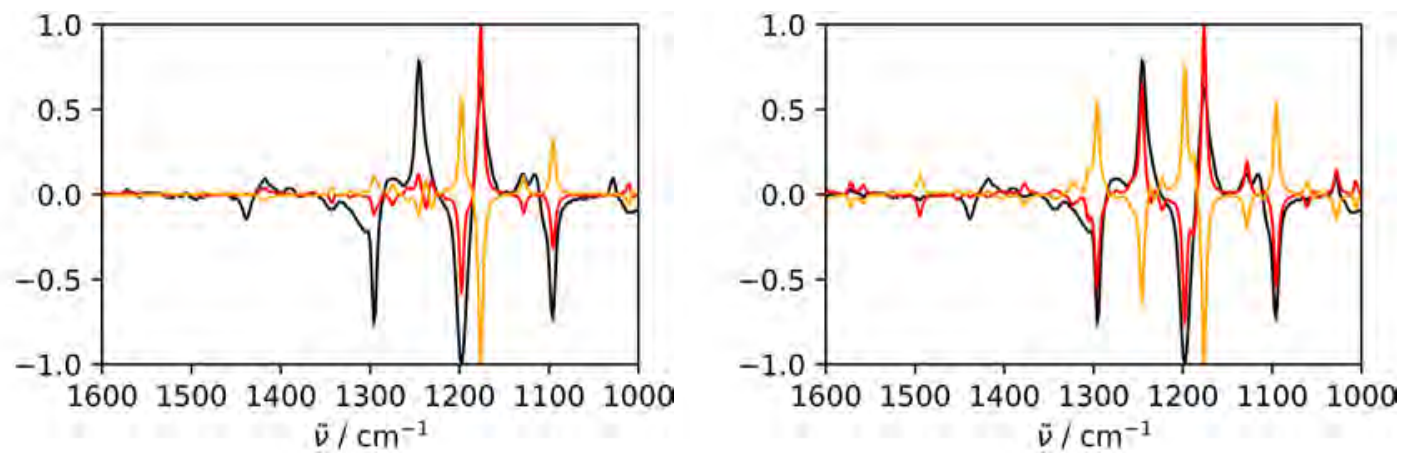

Figure S79: Compound 25, fluralaner. (Left): Aligned theoretical VCD spectrum of the correct enantiomer (red) and the other enantiomer (orange) and experimental VCD spectra (black) using the VSA algorithm, Boltzmann weights based on gas-phase energies. 20 conformers were considered. The alignment was performed in the wavenumber range $[1000,1600] \mathrm{cm}^{-1}$. (Right): Aligned theoretical (red, orange) and experimental VCD spectra (black) using the VSA algorithm with optimized conformer weights. 


\section{References}

[1] Edward C. Sherer, Claire H. Lee, Joseph Shpungin, James F. Cuff, Chenxiao Da, Richard Ball, Richard Bach, Alejandro Crespo, Xiaoyi Gong, and Christopher J. Welch. Systematic approach to conformational sampling for assigning absolute configuration using vibrational circular dichroism. J. Med. Chem., 57:477-494, 2014.

[2] P.J. Stephens and F.J. Devlin. Determination of the structure of chiral molecules using ab initio vibrational circular dichroism spectroscopy. Chirality, 12:172-179, 2000.

[3] Christian Merten, Vangelis Smyrniotopoulos, and Deniz Tasdemir. Assignment of absolute configurations of highly flexible linear diterpenes from the brown algae Bifurcaria bifurcata by ved spectroscopy. Chem. Commun., 51:16217-16220, 2015.

[4] John Kong, Leo A. Joyce, Jinchu Liu, Tiffany M. Jarrell, J. Chris Culberson, and Edward C. Sherer. Absolute configuration assignment of (+)-fluralaner using vibrational circular dichroism. Chirality, 29:854-864, 2017. 